\title{
Antiaromaticity-Aromaticity Interplay in Fused Benzenoid Systems using Molecular Electrostatic Potential Topology
}

\author{
Puthannur K. Anjalikrishna ${ }^{\dagger \ddagger}$, Shridhar R. Gadre and Cherumuttathu H. Suresh ${ }^{\dagger * *}$
}

${ }^{\dagger}$ Chemical Sciences and Technology Division, CSIR-National Institute for Interdisciplinary Science and Technology, Thiruvananthapuram, Kerala, 695019, India

${ }^{\ddagger}$ Academy of Scientific and Innovative Research (AcSIR), Ghaziabad, 201002, India

\$Department of Scientific Computing, Modelling and Simulation, SavitribaiPhule Pune University, Pune, 411007, India

\section{Supporting Information}

\begin{tabular}{|l|l|l|}
\hline \multicolumn{2}{|l|}{ Table of contents } & Page No. \\
\hline Figure S1 & MESP topology of three naphthalene-two cyclobutadiene fused systems. & S2 \\
\hline Figure S2 & $\begin{array}{l}\text { MESP topology and chemical formula of three naphthalene-two } \\
\text { cyclobutadiene fused systems showing fusion of two four-membered } \\
\text { rings on one six-membered ring. }\end{array}$ & S7 \\
\hline Table S1 & $\begin{array}{l}\text { HOMO, LUMO energy and HOMO-LUMO gap of selected set of } \\
\text { systems }\end{array}$ & S8 \\
\hline Table S2 & Cartesian coordinates of all the geometries (in A), SCF energy (in a.u). & S9 \\
\hline
\end{tabular}



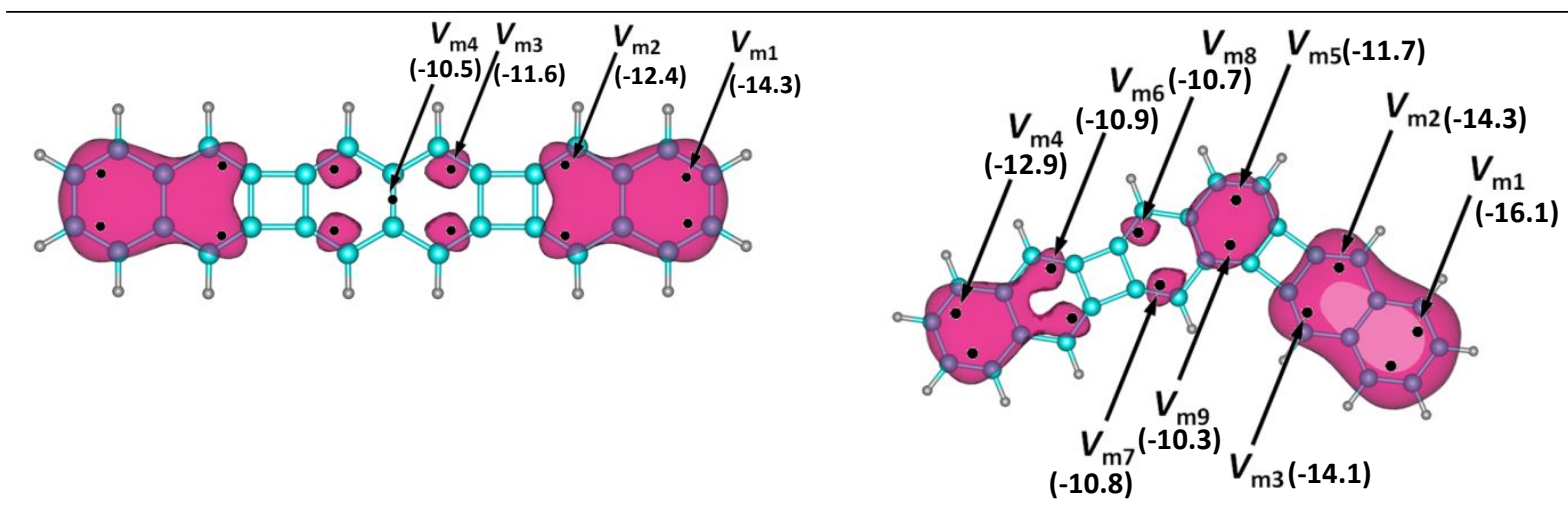

NC7

NC8
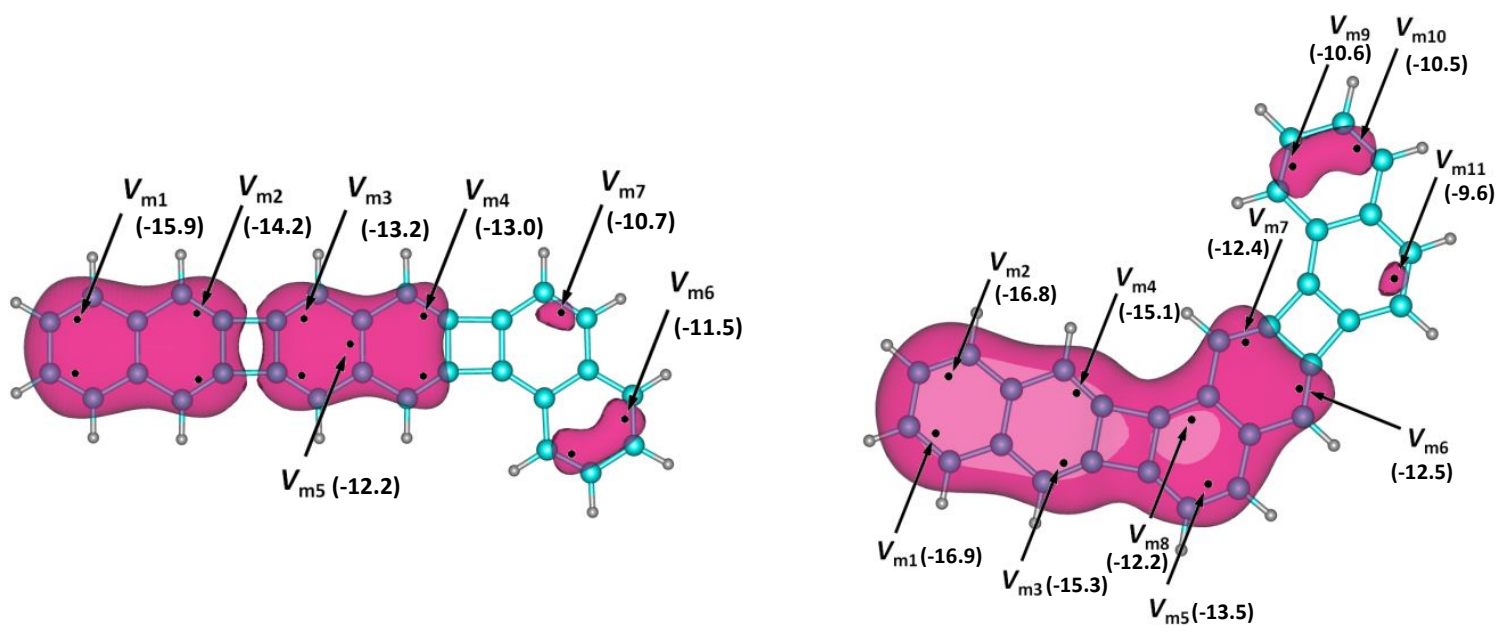

NC9
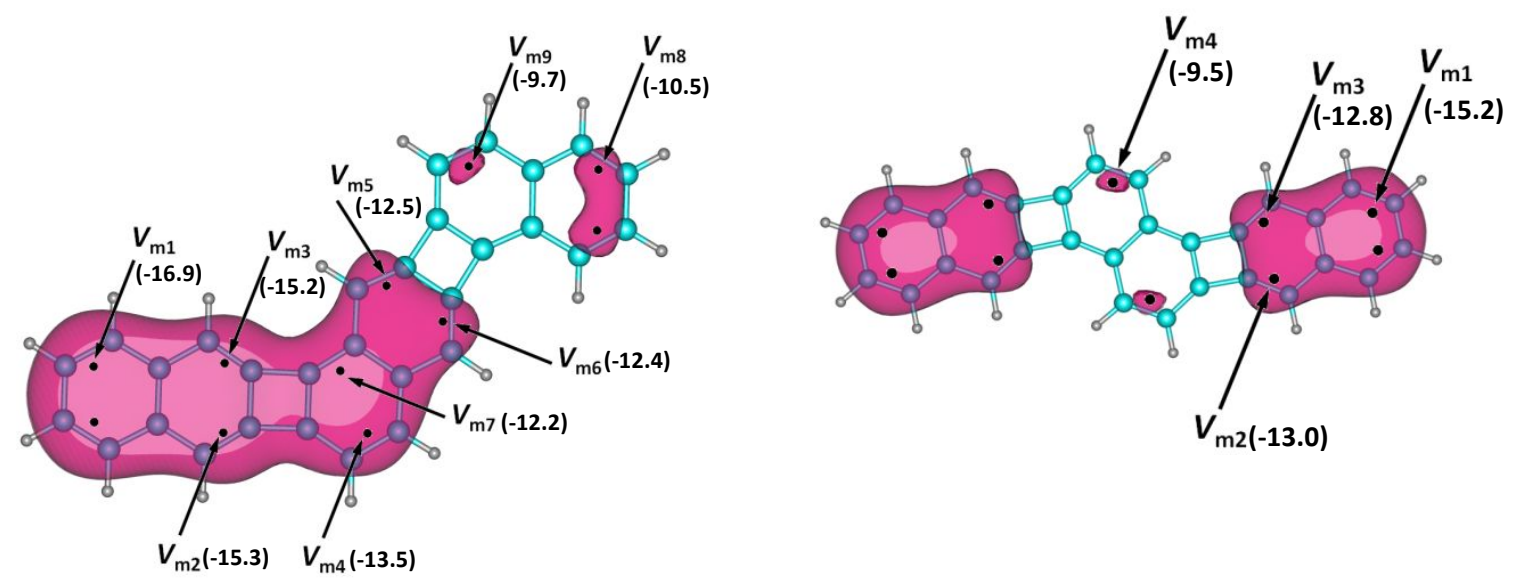

NC11

NC12 


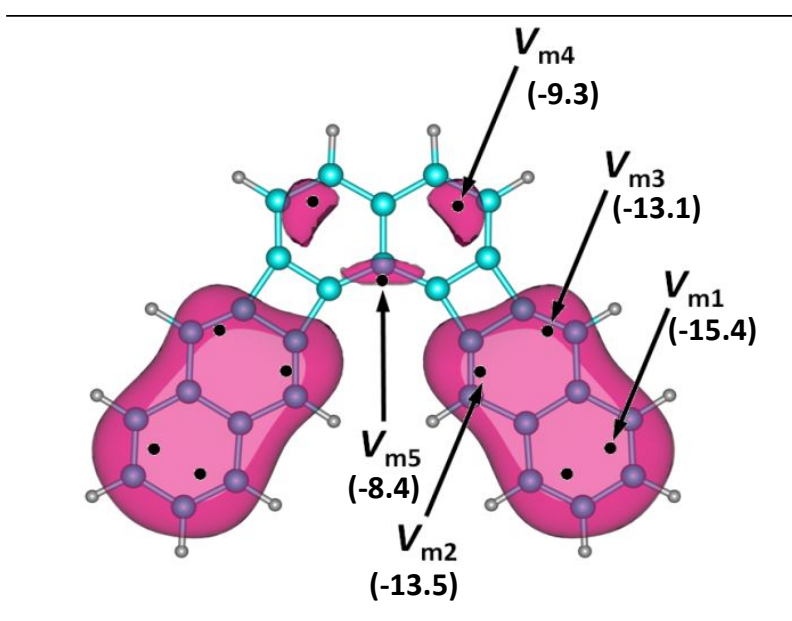

NC13

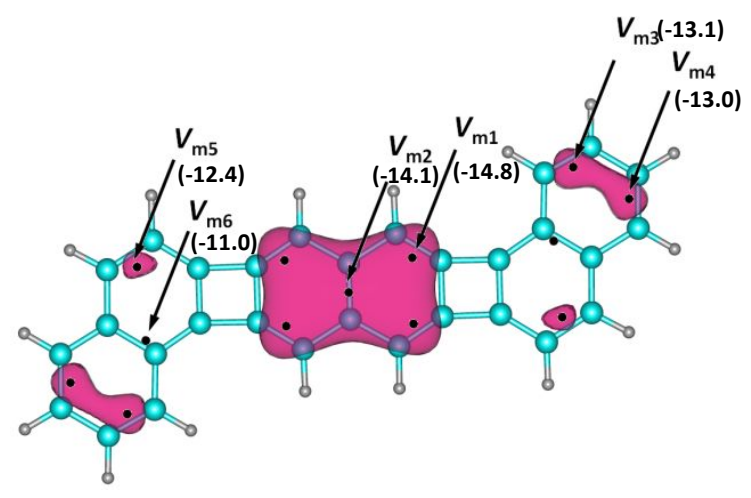

NC15

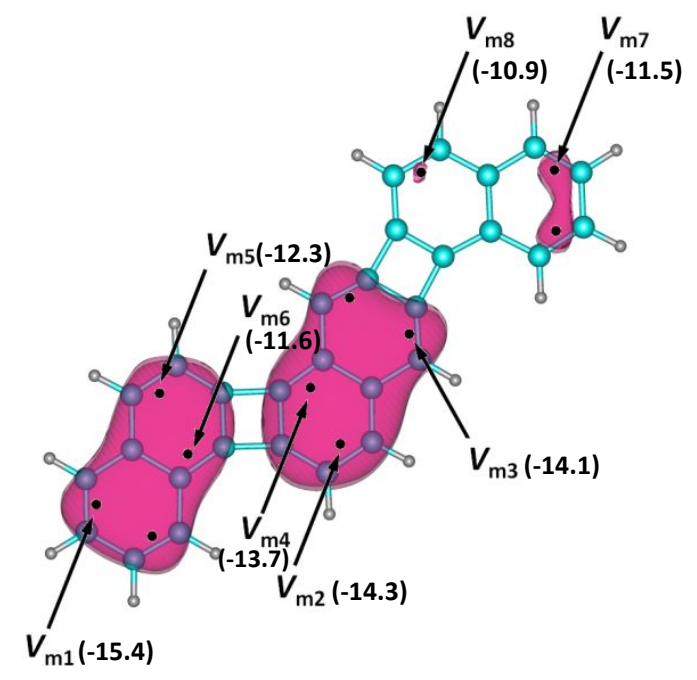

NC17

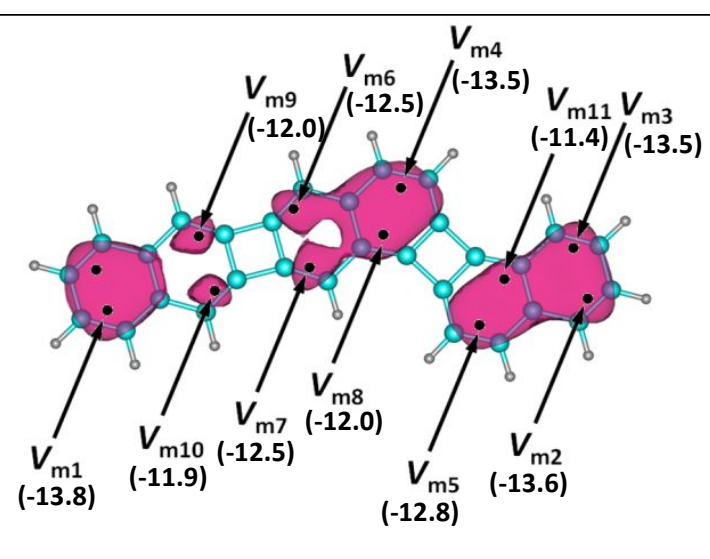

NC14

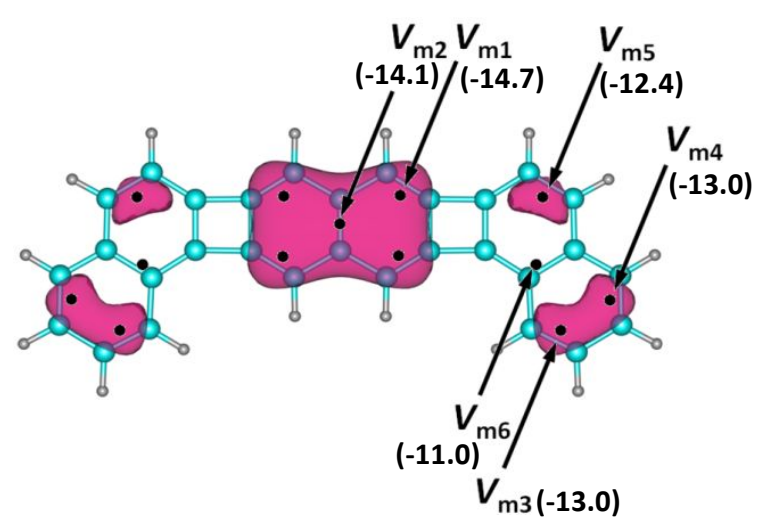

NC16

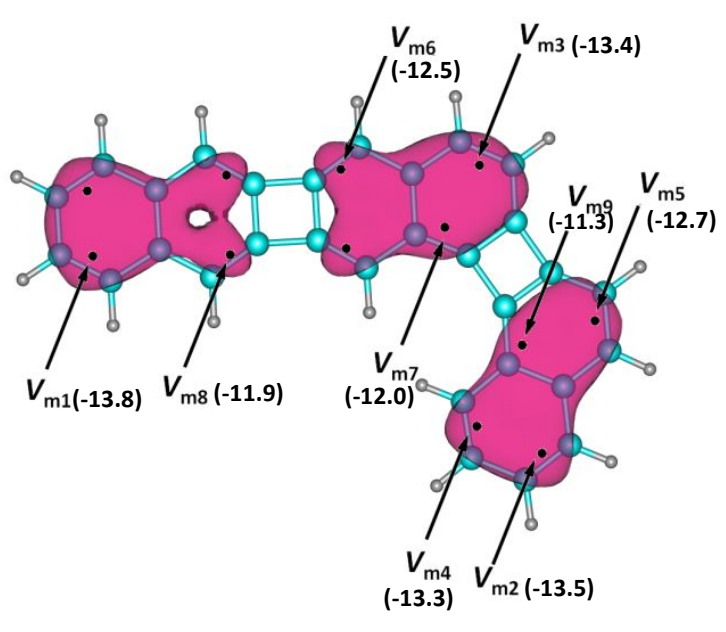

NC18 


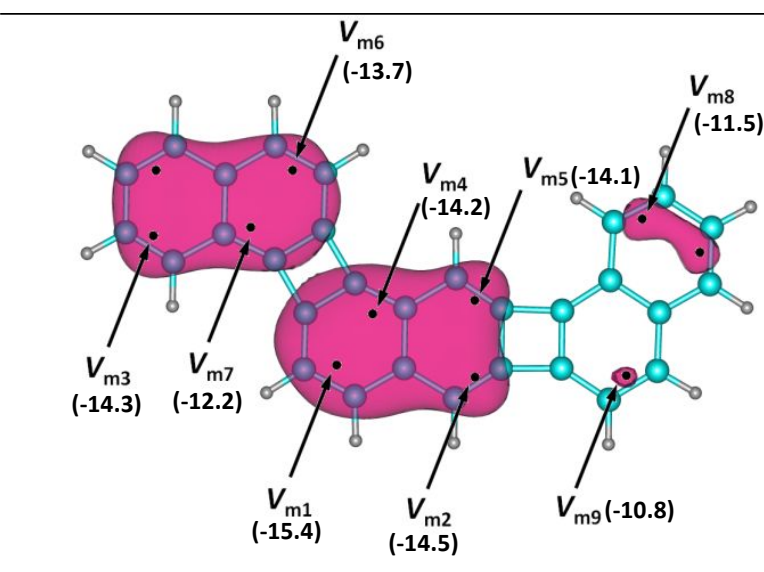

NC19

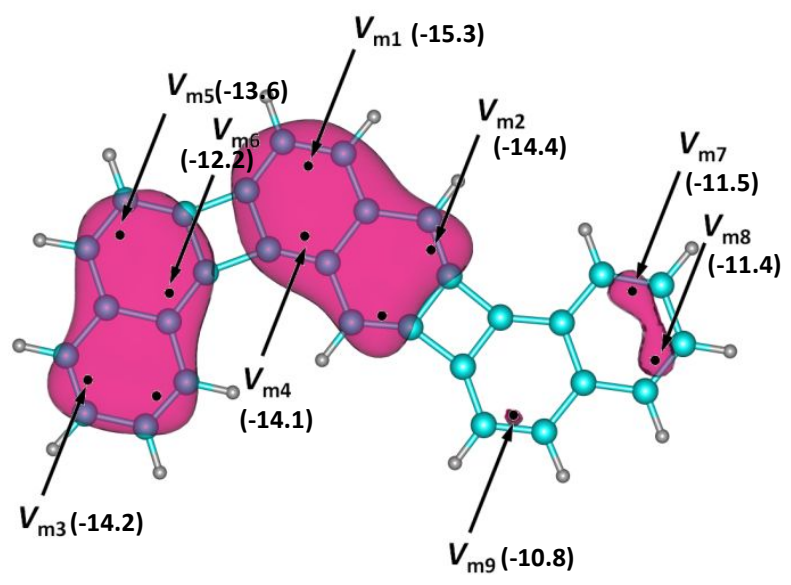

NC21

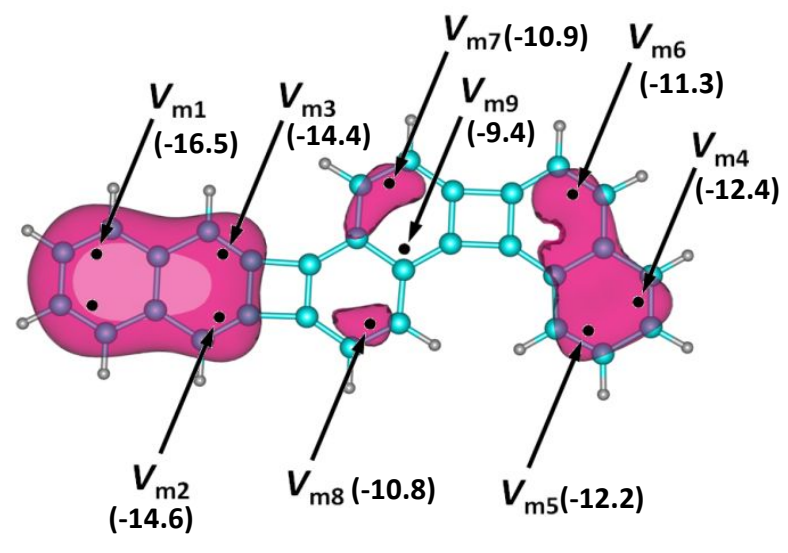

$\mathrm{NC23}$

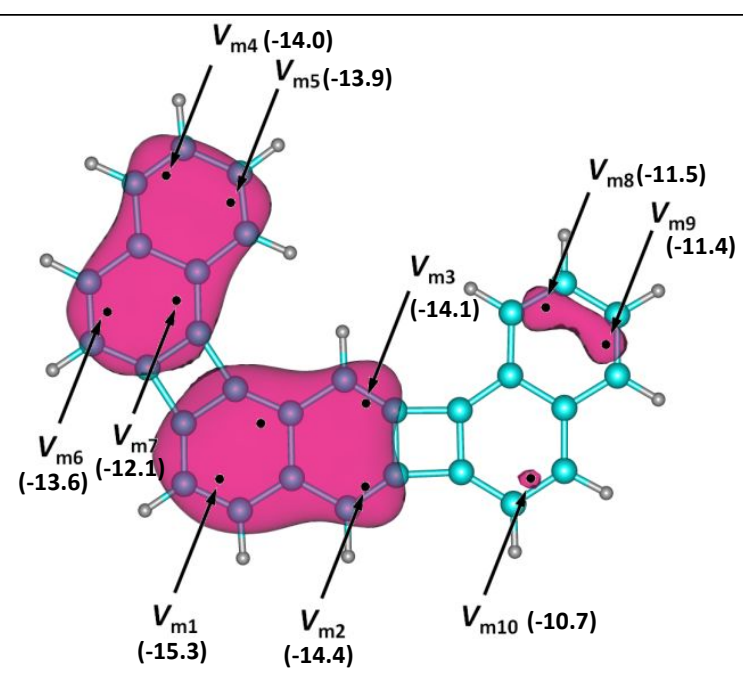

NC20

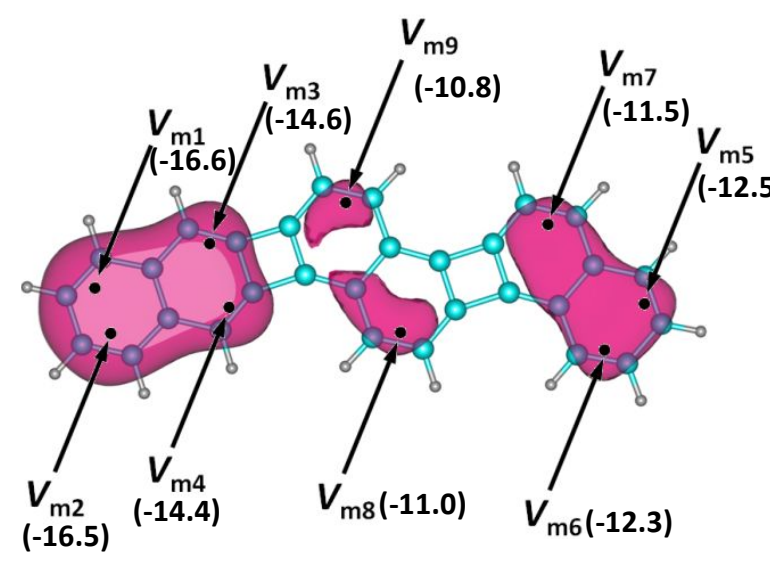

NC22

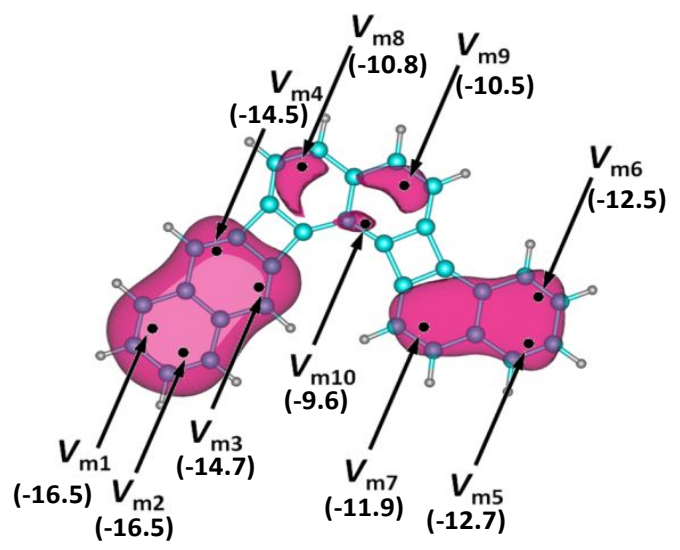

NC24 


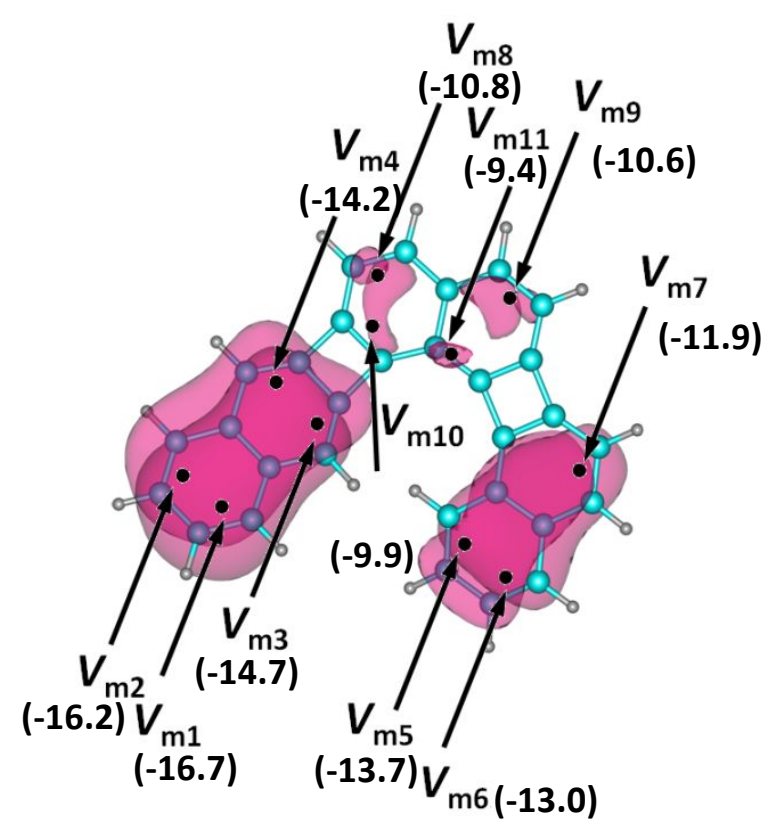

NC25

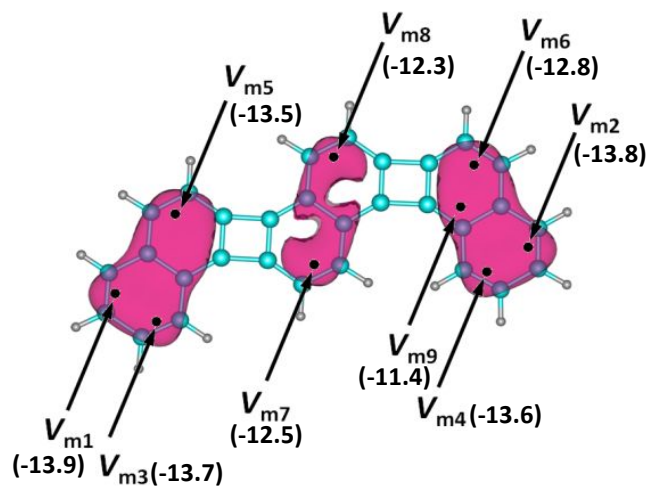

NC27

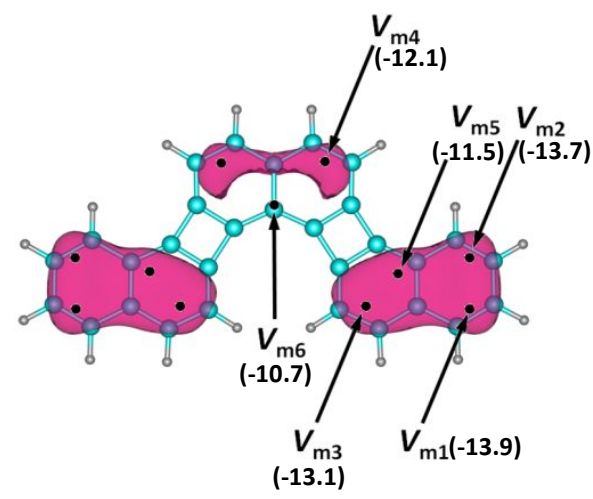

NC29

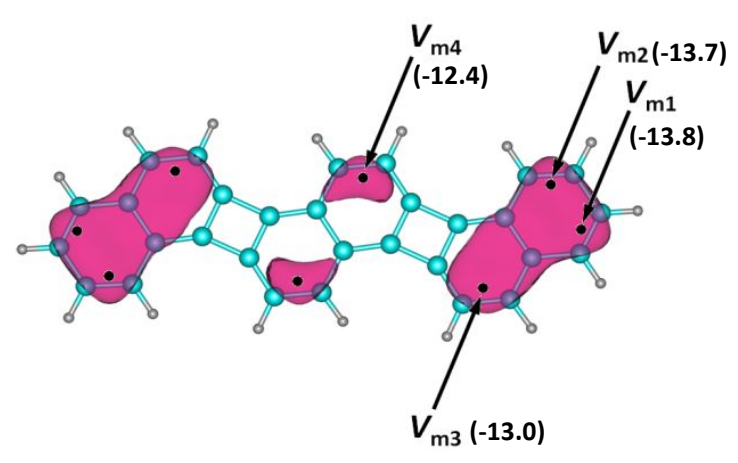

NC26

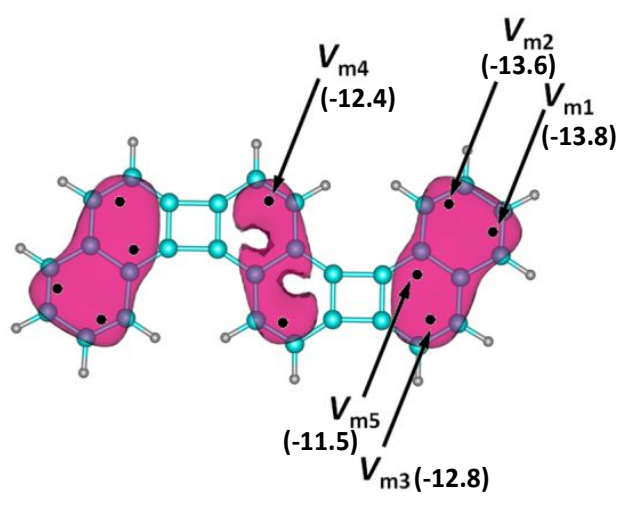

NC28

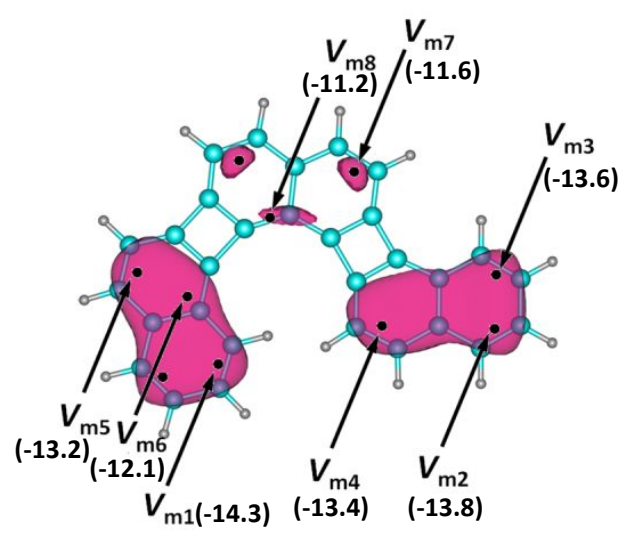

NC30 


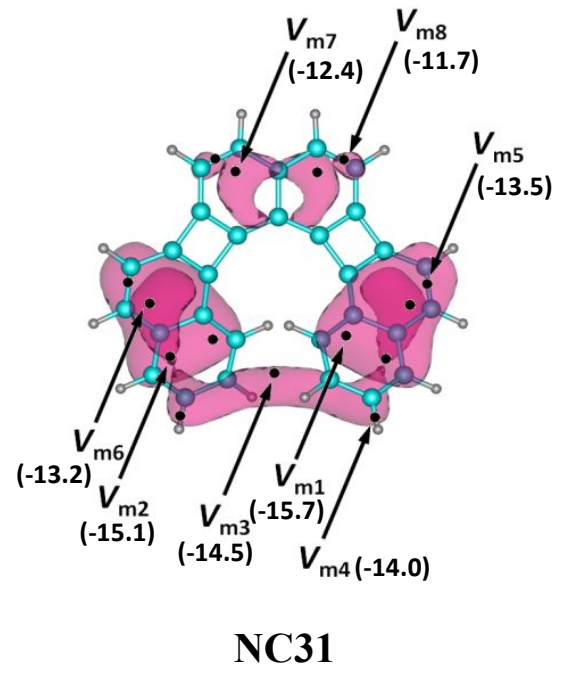

Figure S1. MESP topology of three naphthalene-two cyclobutadiene fused systems. 


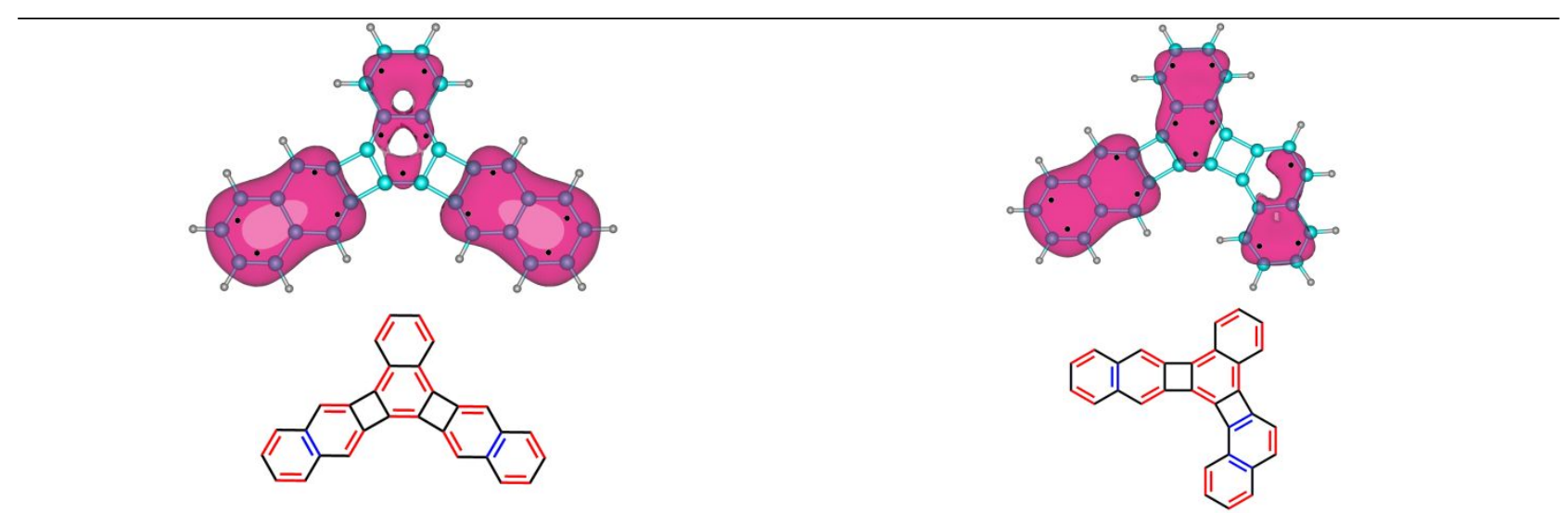

NC33

NC34
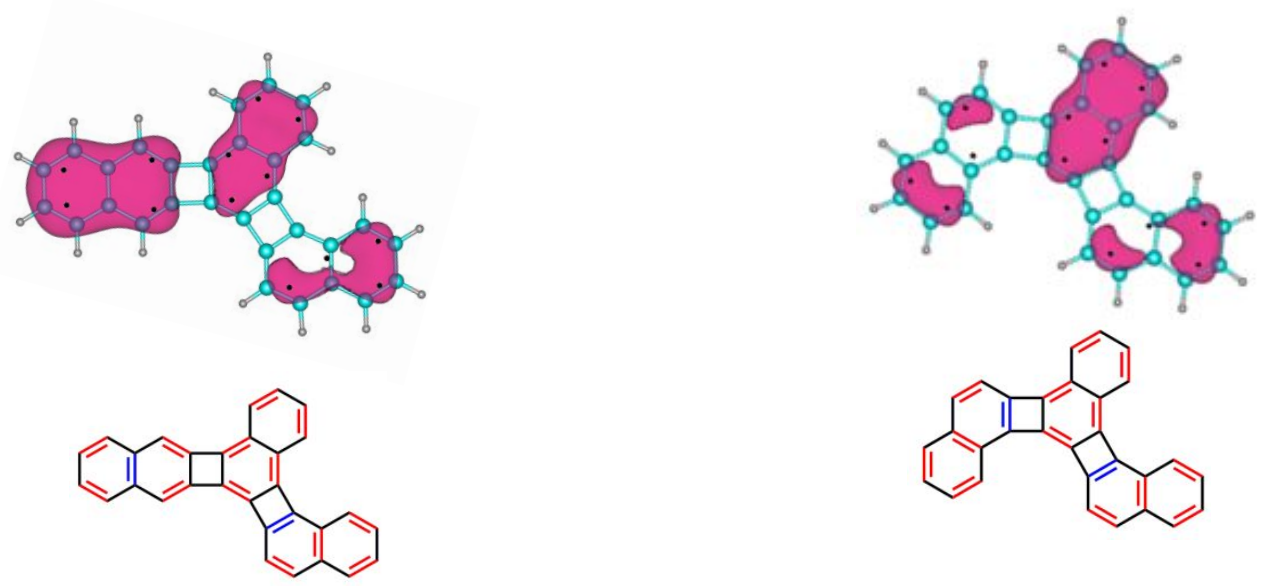

NC34

NC35
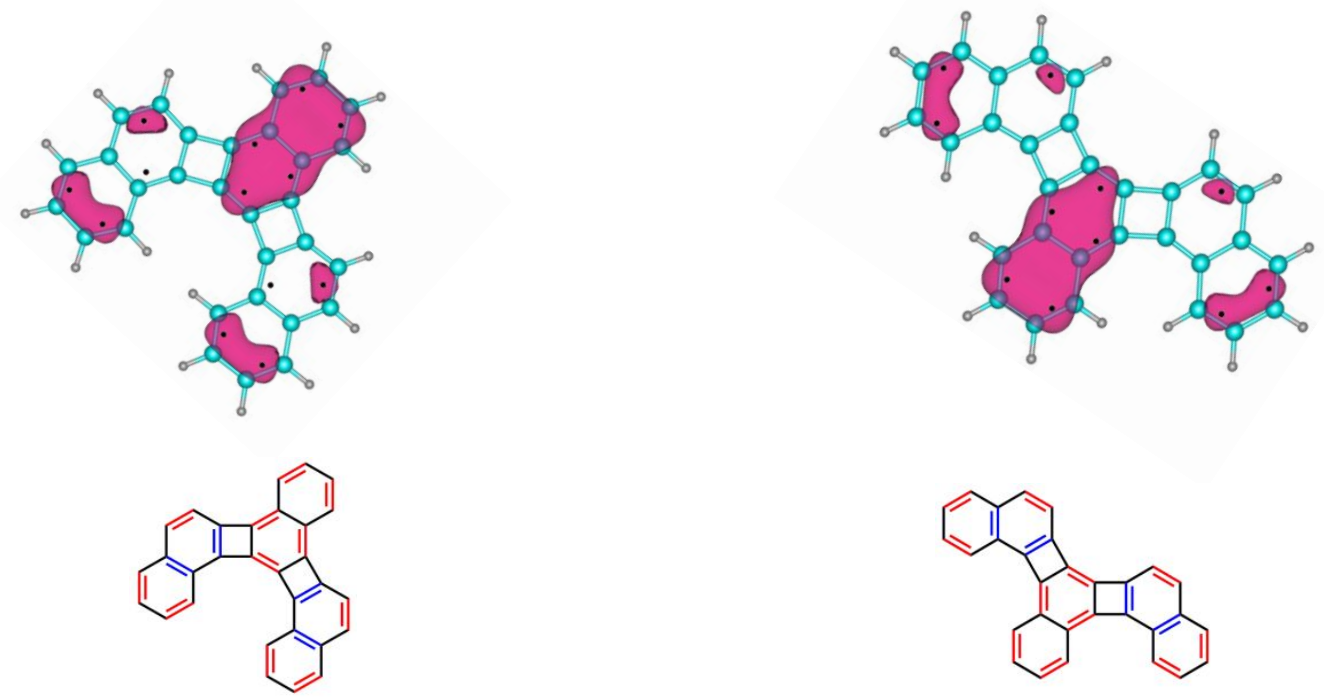

NC36

\section{NC37}

Figure S2. MESP topology and chemical formula of three naphthalene-two cyclobutadiene fused systems showing fusion of two fourmembered rings on one six-membered ring. 
Table S1. Number of HOMO, LUMO energy and HOMO-LUMO gap of selected set of systems

\begin{tabular}{|c|c|c|c|}
\hline Molecule & HOMO & LUMO & $\begin{array}{c}\text { HOMO-LUMO } \\
\text { Energy }\end{array}$ \\
\hline benzene & -7.08 & -0.48 & 6.60 \\
\hline naphthalene & -6.15 & -1.40 & 4.75 \\
\hline phenanthrene & -6.09 & -1.41 & 4.68 \\
\hline pyrene & -5.67 & -1.86 & 3.81 \\
\hline cyclobutadiene & -5.44 & -1.90 & 3.54 \\
\hline pentalene & -5.52 & -2.94 & 2.57 \\
\hline BC1 & -5.62 & -1.88 & 3.74 \\
\hline $\mathrm{BC} 2$ & -5.72 & -1.65 & 4.07 \\
\hline $\mathrm{BC} 3$ & -5.13 & -2.16 & 2.97 \\
\hline $\mathrm{BC} 4$ & -5.63 & -1.91 & 3.72 \\
\hline BP1 & -5.62 & -2.81 & 2.81 \\
\hline BP2 & -5.76 & -2.66 & 3.10 \\
\hline $\mathrm{BP} 3$ & -5.03 & -3.34 & 1.68 \\
\hline $\mathrm{NC} 1$ & -5.74 & -1.90 & 3.85 \\
\hline $\mathrm{NC} 2$ & -5.20 & -2.19 & 3.01 \\
\hline NC3 & -5.91 & -2.01 & 3.90 \\
\hline $\mathrm{NC4}$ & -5.51 & -2.05 & 3.46 \\
\hline NC5 & -5.12 & -2.26 & 2.86 \\
\hline NC6 & -5.12 & -2.29 & 2.83 \\
\hline $\mathrm{NC7}$ & -5.55 & -2.21 & 3.34 \\
\hline NC8 & -5.50 & -2.30 & 3.20 \\
\hline NC9 & -5.28 & -2.20 & 3.08 \\
\hline $\mathrm{NC} 10$ & -5.45 & -2.23 & 3.22 \\
\hline $\mathrm{NC} 11$ & -5.43 & -2.22 & 3.21 \\
\hline $\mathrm{NC} 12$ & -5.30 & -2.42 & 2.88 \\
\hline $\mathrm{NC} 13$ & -5.32 & -2.41 & 2.91 \\
\hline $\mathrm{NC} 14$ & -5.14 & -2.39 & 2.75 \\
\hline $\mathrm{NC} 15$ & -5.04 & -2.27 & 2.77 \\
\hline $\mathrm{NC} 16$ & -5.04 & -2.26 & 2.78 \\
\hline $\mathrm{NC} 17$ & -5.14 & -2.41 & 2.73 \\
\hline $\mathrm{NC} 18$ & -5.11 & -2.18 & 2.93 \\
\hline NC19 & -5.10 & -2.24 & 2.87 \\
\hline $\mathrm{NC} 20$ & -5.12 & -2.20 & 2.92 \\
\hline $\mathrm{NC} 21$ & -5.11 & -2.22 & 2.89 \\
\hline $\mathrm{NC} 22$ & -5.02 & -2.53 & 2.49 \\
\hline $\mathrm{NC} 23$ & -5.03 & -2.56 & 2.47 \\
\hline $\mathrm{NC} 24$ & -5.05 & -2.53 & 2.52 \\
\hline $\mathrm{NC} 25$ & -5.04 & -2.55 & 2.49 \\
\hline
\end{tabular}




\begin{tabular}{cccc}
\hline Molecule & HOMO & LUMO & $\begin{array}{c}\text { HOMO-LUMO } \\
\text { Energy }\end{array}$ \\
\hline NC26 & -4.79 & -2.59 & 2.20 \\
NC27 & -4.79 & -2.60 & 2.19 \\
NC28 & -4.79 & -2.61 & 2.18 \\
NC29 & -4.83 & -2.57 & 2.26 \\
NC30 & -4.84 & -2.59 & 2.25 \\
NC31 & -4.81 & -2.61 & 2.19 \\
NP1 & -5.62 & -2.76 & 2.86 \\
NP2 & -5.38 & -2.94 & 2.44 \\
NP3 & -5.56 & -2.54 & 3.02 \\
NP4 & -5.50 & -2.77 & 2.74 \\
NP5 & -5.33 & -2.92 & 2.40 \\
NP6 & -5.31 & -2.94 & 2.37 \\
NP7 & -5.31 & -2.96 & 2.35 \\
BC5 & -5.35 & -2.59 & 2.76 \\
NC32 & -4.38 & -2.57 & 1.82 \\
PhC1 & -5.25 & -1.97 & 3.27 \\
PyC1 & -4.63 & -2.68 & 1.94 \\
\hline
\end{tabular}

Table S2. Cartesian coordinates of all the geometries (in $\AA$ ), and SCF energy (in a.u).

Coordinates of the molecules (Given in the order Atomic number (At.No.), x coordinate, y coordinate, $\mathrm{z}$ coordinate)

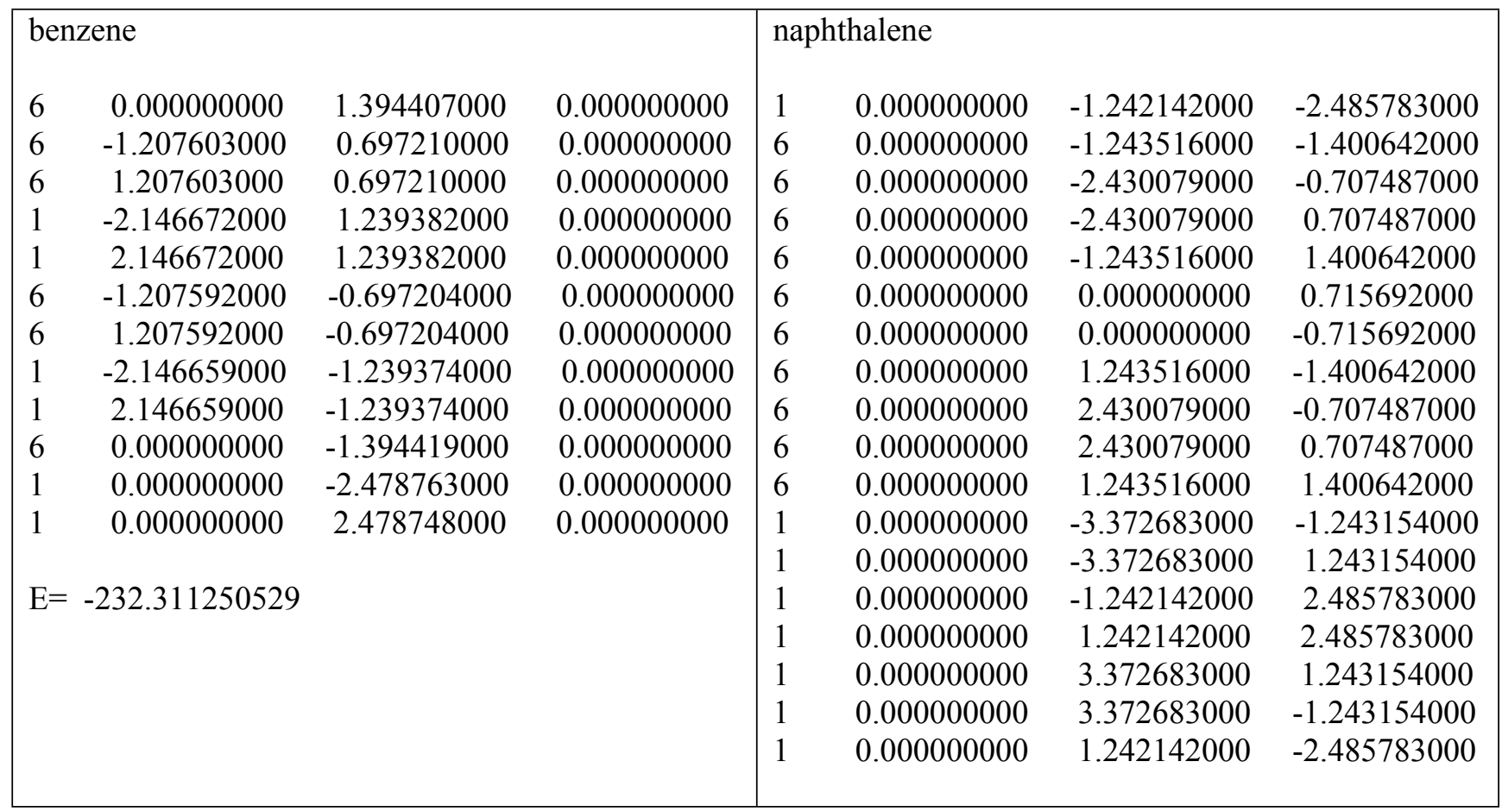




\begin{tabular}{|c|c|c|c|c|c|c|c|}
\hline & & & & & -385.988871103 & & \\
\hline & lathrene & & & & & & \\
\hline 6 & 0.000000000 & -2.834143000 & 0.877241000 & 6 & 0.000000000 & 2.460935000 & 0.679527000 \\
\hline 6 & 0.000000000 & -3.556452000 & -0.296782000 & 6 & 0.000000000 & 1.234414000 & 1.427126000 \\
\hline 6 & 0.000000000 & -1.421171000 & 0.865138000 & 6 & 0.000000000 & 2.460935000 & -0.679527000 \\
\hline 6 & 0.000000000 & -2.878166000 & -1.527682000 & 6 & 0.000000000 & 0.000000000 & 0.712479000 \\
\hline 6 & 0.000000000 & -0.728116000 & -0.379465000 & 6 & 0.000000000 & 1.234414000 & -1.427126000 \\
\hline 6 & 0.000000000 & -1.497791000 & -1.564416000 & 6 & 0.000000000 & 0.000000000 & -0.712479000 \\
\hline 6 & 0.000000000 & -0.678578000 & 2.091763000 & 1 & 0.000000000 & 3.397715000 & 1.226999000 \\
\hline 6 & 0.000000000 & 0.678578000 & 2.091763000 & 6 & 0.000000000 & 1.208903000 & 2.829325000 \\
\hline 6 & 0.000000000 & 1.421171000 & 0.865138000 & 6 & 0.000000000 & -1.234414000 & 1.427126000 \\
\hline 6 & 0.000000000 & 0.728116000 & -0.379465000 & 6 & 0.000000000 & -1.234414000 & -1.427126000 \\
\hline 1 & 0.000000000 & -3.345580000 & 1.834258000 & 6 & 0.000000000 & -1.208903000 & 2.829325000 \\
\hline 1 & 0.000000000 & -4.640179000 & -0.272919000 & 6 & 0.000000000 & 0.000000000 & 3.519003000 \\
\hline 1 & 0.000000000 & -3.440408000 & -2.454660000 & 1 & 0.000000000 & 2.146250000 & 3.375526000 \\
\hline 1 & 0.000000000 & -1.005590000 & -2.528269000 & 6 & 0.000000000 & -2.460935000 & 0.679527000 \\
\hline 1 & 0.000000000 & -1.228909000 & 3.026810000 & 1 & 0.000000000 & -2.146250000 & 3.375526000 \\
\hline 1 & 0.000000000 & 1.228909000 & 3.026810000 & 1 & 0.000000000 & 0.000000000 & 4.603195000 \\
\hline 6 & 0.000000000 & 2.834143000 & 0.877241000 & 6 & 0.000000000 & 1.208903000 & -2.829325000 \\
\hline 6 & 0.000000000 & 1.497791000 & -1.564416000 & 6 & 0.000000000 & -1.208903000 & -2.829325000 \\
\hline 6 & 0.000000000 & 2.878166000 & -1.527682000 & 6 & 0.000000000 & 0.000000000 & -3.519003000 \\
\hline 6 & 0.000000000 & 3.556452000 & -0.296782000 & 1 & 0.000000000 & 3.397715000 & -1.226999000 \\
\hline 1 & 0.000000000 & 3.345580000 & 1.834258000 & 6 & 0.000000000 & -2.460935000 & -0.679527000 \\
\hline 1 & 0.000000000 & 1.005590000 & -2.528269000 & 1 & 0.000000000 & -3.397715000 & 1.226999000 \\
\hline 1 & 0.000000000 & 3.440408000 & -2.454660000 & 1 & 0.000000000 & -2.146250000 & -3.375526000 \\
\hline 1 & 0.000000000 & 4.640179000 & -0.272919000 & 1 & 0.000000000 & 0.000000000 & -4.603195000 \\
\hline & & & & 1 & 0.000000000 & -3.397715000 & -1.226999000 \\
\hline & 539.668405822 & & & 1 & 0.000000000 & 2.146250000 & -3.375526000 \\
\hline & & & & & -615.915983404 & & \\
\hline & obutadiene & & & & talene & & \\
\hline 6 & 0.000000000 & 0.666688000 & 0.788479000 & 6 & 0.276983000 & -0.014384000 & -1.705054000 \\
\hline 6 & 0.000000000 & -0.666688000 & 0.788479000 & 6 & 0.135737000 & -1.497647000 & -1.565914000 \\
\hline 6 & 0.000000000 & -0.666688000 & -0.788479000 & 6 & -0.100410000 & -1.806992000 & -0.268952000 \\
\hline 6 & 0.000000000 & 0.666688000 & -0.788479000 & 6 & -0.120823000 & -0.536512000 & 0.478229000 \\
\hline 1 & 0.000000000 & 1.431952000 & 1.552201000 & 6 & 0.120823000 & 0.536512000 & -0.478229000 \\
\hline 1 & 0.000000000 & -1.431952000 & 1.552201000 & 6 & -0.276983000 & 0.014384000 & 1.705054000 \\
\hline 1 & 0.000000000 & -1.431952000 & -1.552201000 & 6 & -0.135737000 & 1.497647000 & 1.565914000 \\
\hline & 0.000000000 & 1.431952000 & -1.552201000 & 6 & 0.100410000 & 1.806992000 & 0.268952000 \\
\hline & & & & 1 & 0.469845000 & 0.491699000 & -2.642835000 \\
\hline & 154.721159788 & & & 1 & 0.214766000 & -2.193815000 & -2.389417000 \\
\hline & & & & 1 & -0.246422000 & -2.795248000 & 0.142748000 \\
\hline & & & & 1 & -0.469845000 & -0.491699000 & 2.642835000 \\
\hline & & & & & -0.214766000 & 2.193815000 & 2.389417000 \\
\hline & & & & & 0.246422000 & 2.795248000 & -0.142748000 \\
\hline & & & & & -308.447571708 & & \\
\hline $\mathrm{BC}$ & & & & $\mathrm{BC}$ & & & \\
\hline
\end{tabular}




\begin{tabular}{|c|c|c|c|c|c|c|c|}
\hline 6 & 1.844487000 & 0.688050000 & 0.000053000 & 6 & 3.119346000 & 0.693859000 & 0.000286000 \\
\hline 6 & 1.844487000 & -0.688050000 & -0.000041000 & 6 & 3.119345000 & -0.693859000 & 0.000357000 \\
\hline 6 & 0.622905000 & -1.443402000 & -0.000084000 & 6 & 1.913665000 & -1.443216000 & 0.000036000 \\
\hline 6 & -0.522525000 & -0.711526000 & -0.000049000 & 6 & 0.754287000 & -0.711228000 & -0.000447000 \\
\hline 6 & -0.522525000 & 0.711526000 & -0.000006000 & 6 & 0.754288000 & 0.711228000 & -0.000521000 \\
\hline 6 & 0.622905000 & 1.443402000 & 0.000084000 & 6 & 1.913666000 & 1.443216000 & -0.000109000 \\
\hline 6 & -2.046517000 & -0.675109000 & 0.000120000 & 6 & -0.754288000 & -0.711234000 & -0.000433000 \\
\hline 6 & -2.046518000 & 0.675109000 & -0.000098000 & 6 & -0.754290000 & 0.711233000 & -0.000522000 \\
\hline 1 & 2.788890000 & 1.220731000 & 0.000080000 & 6 & -1.913660000 & -1.443217000 & 0.000107000 \\
\hline 1 & 2.788890000 & -1.220731000 & -0.000053000 & 6 & -3.119349000 & -0.693853000 & 0.000453000 \\
\hline 1 & 0.644671000 & -2.527126000 & -0.000075000 & 6 & -3.119350000 & 0.693854000 & 0.000365000 \\
\hline 1 & 0.644671000 & 2.527126000 & 0.000100000 & 6 & -1.913660000 & 1.443218000 & -0.000077000 \\
\hline 1 & -2.823655000 & -1.426867000 & 0.000246000 & 1 & 4.066858000 & 1.221112000 & 0.000561000 \\
\hline 1 & -2.823655000 & 1.426867000 & -0.000175000 & 1 & 4.066856000 & -1.221114000 & 0.000688000 \\
\hline & & & & 1 & 1.934612000 & -2.526753000 & 0.000176000 \\
\hline & -308.438025917 & & & 1 & 1.934613000 & 2.526753000 & -0.000086000 \\
\hline & & & & 1 & -1.934621000 & -2.526754000 & 0.000259000 \\
\hline & & & & 1 & -4.066854000 & -1.221120000 & 0.000820000 \\
\hline & & & & 1 & -4.066855000 & 1.221119000 & 0.000667000 \\
\hline & & & & 1 & -1.934619000 & 2.526754000 & -0.000055000 \\
\hline & & & & & 462.142413829 & & \\
\hline & & & & $\overline{\mathrm{BC}}$ & & & \\
\hline 6 & 2.664556000 & -0.711685000 & 0.000005000 & 6 & -2.683196000 & -1.875391000 & 0.000001000 \\
\hline 6 & 2.664557000 & 0.711686000 & 0.000010000 & 6 & -4.094538000 & -1.782493000 & 0.000012000 \\
\hline 6 & 1.155924000 & -0.709016000 & -0.000013000 & 6 & -4.750173000 & -0.554500000 & 0.000011000 \\
\hline 6 & 1.155925000 & 0.709020000 & -0.000016000 & 6 & -4.044468000 & 0.671070000 & 0.000001000 \\
\hline 6 & 3.821074000 & 1.443497000 & 0.000015000 & 6 & -2.671989000 & 0.575841000 & -0.000010000 \\
\hline 6 & 3.821071000 & -1.443498000 & 0.000005000 & 6 & -2.003038000 & -0.680609000 & -0.000015000 \\
\hline 6 & 5.030709000 & 0.692311000 & 0.000021000 & 6 & -1.358437000 & 1.298199000 & -0.000007000 \\
\hline 6 & 5.030708000 & -0.692314000 & 0.000016000 & 6 & -0.675958000 & 0.012103000 & -0.000004000 \\
\hline 6 & -0.000004000 & -1.485868000 & -0.000019000 & 6 & -0.723412000 & 2.504108000 & -0.000001000 \\
\hline 6 & -1.155924000 & -0.709024000 & -0.000046000 & 6 & 0.723423000 & 2.504130000 & 0.000004000 \\
\hline 6 & -1.155924000 & 0.709027000 & -0.000049000 & 6 & 1.358461000 & 1.298212000 & 0.000000000 \\
\hline 6 & -2.664555000 & -0.711681000 & -0.000005000 & 6 & 0.675969000 & 0.012113000 & -0.000008000 \\
\hline 6 & -2.664555000 & 0.711681000 & -0.000006000 & 6 & 2.671999000 & 0.575846000 & -0.000006000 \\
\hline 6 & -3.821076000 & -1.443498000 & 0.000012000 & 6 & 2.003023000 & -0.680596000 & -0.000011000 \\
\hline 6 & -0.000005000 & 1.485870000 & -0.000027000 & 6 & 4.044483000 & 0.671054000 & 0.000007000 \\
\hline 6 & -3.821077000 & 1.443497000 & 0.000009000 & 6 & 4.750170000 & -0.554524000 & 0.000011000 \\
\hline 6 & -5.030704000 & -0.692318000 & 0.000033000 & 6 & 4.094512000 & -1.782509000 & 0.000006000 \\
\hline 6 & -5.030704000 & 0.692315000 & 0.000032000 & 6 & 2.683173000 & -1.875388000 & -0.000003000 \\
\hline 1 & 3.842313000 & 2.526982000 & 0.000013000 & 1 & -2.189882000 & -2.840250000 & 0.000004000 \\
\hline 1 & 5.977430000 & 1.220873000 & 0.000028000 & 1 & -4.683185000 & -2.693090000 & 0.000022000 \\
\hline 1 & 5.977428000 & -1.220879000 & 0.000021000 & 1 & -5.834413000 & -0.538088000 & 0.000019000 \\
\hline 1 & 3.842307000 & -2.526983000 & -0.000001000 & 1 & -4.574628000 & 1.616271000 & 0.000005000 \\
\hline 1 & 0.000006000 & -2.568928000 & -0.000011000 & 1 & -1.260981000 & 3.445186000 & -0.000003000 \\
\hline 1 & -3.842301000 & -2.526983000 & 0.000012000 & 1 & 1.261002000 & 3.445196000 & 0.000009000 \\
\hline 1 & -5.977430000 & -1.220872000 & 0.000049000 & 1 & 4.574651000 & 1.616252000 & 0.000014000 \\
\hline 1 & -5.977430000 & 1.220868000 & 0.000047000 & 1 & 5.834409000 & -0.538134000 & 0.000019000 \\
\hline 1 & -3.842306000 & 2.526982000 & 0.000007000 & 1 & 4.683154000 & -2.693109000 & 0.000010000 \\
\hline
\end{tabular}




\begin{tabular}{|c|c|c|c|c|c|c|c|}
\hline $\mathrm{E}=$ & $\begin{array}{r}0.000002000 \\
-691.974574696 \\
\end{array}$ & 2.568931000 & -0.000022000 & $E=$ & $\begin{array}{r}2.189841000 \\
691.978930672 \\
\end{array}$ & -2.840239000 & -0.000004000 \\
\hline \multicolumn{4}{|c|}{ BP1 } & \multicolumn{4}{|c|}{ BP2 } \\
\hline 6 & -2.929746000 & 0.769727000 & 0.000288000 & 6 & 3.559962000 & -0.230551000 & 0.000000000 \\
\hline 6 & -3.276537000 & -0.544077000 & -0.000302000 & 6 & 2.180728000 & -0.088549000 & 0.000000000 \\
\hline 6 & -1.467562000 & 0.815881000 & -0.000003000 & 6 & 1.336493000 & -1.239602000 & 0.000000000 \\
\hline 6 & -0.981386000 & -0.568962000 & 0.000013000 & 6 & 1.886842000 & -2.510071000 & 0.000000000 \\
\hline 6 & -2.057232000 & -1.393056000 & 0.000240000 & 6 & 3.285118000 & -2.644972000 & 0.000000000 \\
\hline 6 & -0.412737000 & 1.666927000 & -0.000075000 & 6 & 4.108560000 & -1.523055000 & 0.000000000 \\
\hline 6 & 0.833755000 & 0.866999000 & -0.000042000 & 6 & 1.336493000 & 1.122840000 & 0.000000000 \\
\hline 6 & 0.486587000 & -0.517503000 & -0.000019000 & 6 & 0.037484000 & 0.734888000 & 0.000000000 \\
\hline 6 & 2.158502000 & 1.263307000 & -0.000045000 & 6 & -0.037484000 & -0.734888000 & 0.000000000 \\
\hline 6 & 3.162717000 & 0.276262000 & -0.000041000 & 1 & 4.209117000 & 0.638663000 & 0.000000000 \\
\hline 6 & 2.826047000 & -1.070330000 & -0.000019000 & 1 & 1.255118000 & -3.391687000 & 0.000000000 \\
\hline 6 & 1.477597000 & -1.479702000 & -0.000005000 & 1 & 3.726225000 & -3.635275000 & 0.000000000 \\
\hline 1 & -3.600209000 & 1.616555000 & 0.000410000 & 1 & 5.185572000 & -1.647227000 & 0.000000000 \\
\hline 1 & -4.285098000 & -0.935494000 & -0.000535000 & 1 & 1.726794000 & 2.132729000 & 0.000000000 \\
\hline 1 & -2.069411000 & -2.475036000 & 0.000330000 & 6 & -1.336493000 & 1.239602000 & 0.000000000 \\
\hline 1 & -0.437589000 & 2.749922000 & -0.000046000 & 6 & -1.336493000 & -1.122840000 & 0.000000000 \\
\hline 1 & 2.426249000 & 2.314650000 & -0.000060000 & 6 & -1.886842000 & 2.510071000 & 0.000000000 \\
\hline 1 & 4.205466000 & 0.572099000 & -0.000046000 & 6 & -3.285118000 & 2.644972000 & 0.000000000 \\
\hline 1 & 3.609466000 & -1.819835000 & -0.000009000 & 1 & -3.726225000 & 3.635275000 & 0.000000000 \\
\hline \multirow[t]{2}{*}{1} & 1.231105000 & -2.535704000 & 0.000018000 & 6 & -4.108560000 & 1.523055000 & 0.000000000 \\
\hline & & & & 6 & -2.180728000 & 0.088549000 & 0.000000000 \\
\hline \multirow[t]{6}{*}{$\mathrm{E}=$} & -462.150995479 & & & 1 & -5.185572000 & 1.647227000 & 0.000000000 \\
\hline & & & & 6 & -3.559962000 & 0.230551000 & 0.000000000 \\
\hline & & & & 1 & -4.209117000 & -0.638663000 & 0.000000000 \\
\hline & & & & 1 & -1.255118000 & 3.391687000 & 0.000000000 \\
\hline & & & & 1 & -1.726794000 & -2.132729000 & 0.000000000 \\
\hline & & & & \multicolumn{4}{|c|}{$E=-615.850638631$} \\
\hline \multicolumn{4}{|c|}{$\overline{\mathrm{BP} 3}$} & \multicolumn{4}{|c|}{$\mathrm{NC1}$} \\
\hline 6 & -3.637290000 & 0.007069000 & 0.000000000 & 6 & -3.277237000 & -0.679120000 & 0.000180000 \\
\hline 6 & -2.297297000 & 0.353159000 & 0.000000000 & 6 & -3.277239000 & 0.679108000 & 0.000191000 \\
\hline 6 & -1.272008000 & -0.649433000 & 0.000000000 & 6 & -1.774753000 & 0.724772000 & -0.000228000 \\
\hline 6 & -1.629676000 & -1.986118000 & 0.000000000 & 6 & -1.774744000 & -0.724776000 & -0.000264000 \\
\hline 6 & -2.997230000 & -2.329327000 & 0.000000000 & 6 & -0.645283000 & 1.454841000 & -0.000025000 \\
\hline 6 & -3.987713000 & -1.355860000 & 0.000000000 & 6 & 0.612460000 & 0.716722000 & -0.000038000 \\
\hline 6 & -1.653019000 & 1.672542000 & 0.000000000 & 6 & 0.612469000 & -0.716694000 & -0.000023000 \\
\hline 6 & -0.298310000 & 1.489894000 & 0.000000000 & 6 & -0.645273000 & -1.454840000 & -0.000059000 \\
\hline 6 & 0.000000000 & 0.069866000 & 0.000000000 & 6 & 1.844521000 & 1.390706000 & -0.000017000 \\
\hline 1 & -4.408318000 & 0.770496000 & 0.000000000 & 6 & 3.048455000 & 0.699221000 & 0.000033000 \\
\hline 1 & -0.878915000 & -2.767929000 & 0.000000000 & 6 & 3.048460000 & -0.699235000 & 0.000021000 \\
\hline 1 & -3.278103000 & -3.376827000 & 0.000000000 & 6 & 1.844518000 & -1.390707000 & 0.000014000 \\
\hline 1 & -5.032019000 & -1.644676000 & 0.000000000 & 1 & -4.056775000 & -1.428542000 & 0.000447000 \\
\hline 6 & 0.948508000 & 2.239012000 & 0.000000000 & 1 & -4.056759000 & 1.428550000 & 0.000504000 \\
\hline 6 & 1.370561000 & -0.070151000 & 0.000000000 & 1 & -0.623225000 & 2.539405000 & 0.000141000 \\
\hline 6 & 1.959647000 & 1.305476000 & 0.000000000 & 1 & -0.623198000 & -2.539402000 & 0.000081000 \\
\hline
\end{tabular}




\begin{tabular}{|c|c|c|c|c|c|c|c|}
\hline 6 & 2.254244000 & -1.194293000 & 0.000000000 & 1 & 1.844964000 & 2.476231000 & 0.000025000 \\
\hline 6 & 3.384655000 & 1.455690000 & 0.000000000 & 1 & 3.985965000 & 1.243721000 & 0.000073000 \\
\hline 6 & 3.601793000 & -0.981716000 & 0.000000000 & 1 & 3.985984000 & -1.243714000 & 0.000001000 \\
\hline 6 & 4.173384000 & 0.345886000 & 0.000000000 & 1 & 1.844915000 & -2.476230000 & 0.000024000 \\
\hline 1 & 3.820416000 & 2.449119000 & 0.000000000 & & & & \\
\hline 1 & 5.252898000 & 0.443751000 & 0.000000000 & $\mathrm{E}=$ & -462.127419509 & & \\
\hline 1 & 4.277786000 & -1.830059000 & 0.000000000 & & & & \\
\hline 1 & 1.854877000 & -2.201857000 & 0.000000000 & & & & \\
\hline 1 & 1.059393000 & 3.313854000 & 0.000000000 & & & & \\
\hline 1 & -2.189520000 & 2.613960000 & 0.000000000 & & & & \\
\hline \multicolumn{8}{|c|}{$E=-615.816176803$} \\
\hline \multicolumn{4}{|c|}{$\mathrm{NC} 2$} & \multicolumn{4}{|c|}{ NC3 } \\
\hline 6 & 1.562342000 & 1.751312000 & -0.000033000 & 6 & 0.746459000 & -0.722116000 & -0.000576000 \\
\hline 6 & 1.988766000 & 0.441866000 & -0.000495000 & 6 & 0.746558000 & 0.722243000 & -0.000527000 \\
\hline 6 & 1.109236000 & -0.642262000 & -0.000616000 & 6 & -0.746530000 & 0.722262000 & -0.000665000 \\
\hline 6 & -0.265723000 & -0.502997000 & -0.000240000 & 6 & -0.746604000 & -0.721930000 & -0.000650000 \\
\hline 6 & -0.741547000 & 0.876603000 & -0.000081000 & 6 & -1.891089000 & 1.452034000 & -0.000556000 \\
\hline 6 & 0.168031000 & 1.947978000 & 0.000024000 & 6 & -3.130274000 & 0.719502000 & -0.000086000 \\
\hline 6 & -1.218197000 & -1.570543000 & -0.000084000 & 6 & -3.130274000 & -0.719585000 & 0.000035000 \\
\hline 6 & -2.558135000 & -1.304256000 & 0.000155000 & 6 & -1.891002000 & -1.451952000 & -0.000329000 \\
\hline 6 & -3.032063000 & 0.041557000 & 0.000212000 & 6 & -4.372081000 & 1.394835000 & 0.000250000 \\
\hline 6 & -2.153852000 & 1.090401000 & 0.000092000 & 6 & -5.566374000 & 0.703636000 & 0.000617000 \\
\hline 6 & 3.166276000 & -0.547683000 & 0.000398000 & 6 & -5.566361000 & -0.703752000 & 0.000733000 \\
\hline 6 & 2.316870000 & -1.592743000 & 0.000220000 & 6 & -4.372059000 & -1.394934000 & 0.000465000 \\
\hline 1 & 2.240529000 & 2.596713000 & 0.000241000 & 6 & 1.890866000 & -1.452001000 & -0.000504000 \\
\hline 1 & -0.227179000 & 2.958320000 & 0.000199000 & 6 & 3.130310000 & -0.719518000 & -0.000394000 \\
\hline 1 & -0.857672000 & -2.593368000 & -0.000174000 & 6 & 3.130428000 & 0.719546000 & -0.000225000 \\
\hline 1 & -3.274217000 & -2.118542000 & 0.000282000 & 6 & 1.890997000 & 1.452140000 & -0.000305000 \\
\hline 1 & -4.100145000 & 0.227480000 & 0.000355000 & 6 & 4.371951000 & -1.394920000 & 0.000111000 \\
\hline 1 & -2.521610000 & 2.111535000 & 0.000140000 & 6 & 5.566401000 & -0.703743000 & 0.000785000 \\
\hline 1 & 4.242951000 & -0.451604000 & 0.001003000 & 6 & 5.566491000 & 0.703504000 & 0.000909000 \\
\hline \multirow[t]{2}{*}{1} & 2.445315000 & -2.665937000 & 0.000635000 & 6 & 4.372113000 & 1.394826000 & 0.000374000 \\
\hline & & & & 1 & -1.911384000 & 2.536347000 & -0.000580000 \\
\hline & -462.108273656 & & & 1 & -1.910968000 & -2.536269000 & -0.000240000 \\
\hline & & & & 1 & -4.372449000 & 2.480040000 & 0.000151000 \\
\hline & & & & 1 & -6.506206000 & 1.244021000 & 0.000829000 \\
\hline & & & & 1 & -6.506178000 & -1.244164000 & 0.001025000 \\
\hline & & & & 1 & -4.372410000 & -2.480141000 & 0.000534000 \\
\hline & & & & 1 & 1.911183000 & -2.536309000 & -0.000897000 \\
\hline & & & & 1 & 1.911354000 & 2.536450000 & -0.000892000 \\
\hline & & & & 1 & 4.372376000 & -2.480120000 & 0.000051000 \\
\hline & & & & 1 & 6.506146000 & -1.244285000 & 0.001231000 \\
\hline & & & & 1 & 6.506298000 & 1.243941000 & 0.001510000 \\
\hline & & & & & 4.372679000 & 2.480023000 & 0.000510000 \\
\hline & & & & & -769.509183747 & & \\
\hline \multicolumn{4}{|c|}{$\mathrm{NC4}$} & \multicolumn{4}{|c|}{ NC5 } \\
\hline 6 & -0.757090000 & 1.440064000 & 0.000030000 & 6 & 0.324376000 & -0.981528000 & -0.000267000 \\
\hline
\end{tabular}




\begin{tabular}{|c|c|c|c|c|c|c|c|}
\hline 6 & -1.058441000 & 0.072583000 & 0.000013000 & 6 & 0.984770000 & -2.200966000 & -0.000095000 \\
\hline 6 & 0.399263000 & -0.275679000 & -0.000036000 & 6 & 1.002432000 & 0.243453000 & -0.000281000 \\
\hline 6 & 0.712554000 & 1.139140000 & -0.000055000 & 6 & 2.389653000 & 0.342678000 & -0.000093000 \\
\hline 6 & 1.354053000 & -1.232441000 & 0.000001000 & 6 & 3.094783000 & -0.927011000 & 0.000036000 \\
\hline 6 & 2.731584000 & -0.783302000 & -0.000006000 & 6 & 2.381872000 & -2.146926000 & 0.000029000 \\
\hline 6 & 3.041110000 & 0.618179000 & -0.000014000 & 6 & 3.136745000 & 1.557201000 & -0.000044000 \\
\hline 6 & 1.980175000 & 1.605420000 & -0.000013000 & 6 & 4.505596000 & 1.530244000 & 0.000125000 \\
\hline 6 & 3.793608000 & -1.709190000 & 0.000008000 & 6 & 5.204411000 & 0.291074000 & 0.000246000 \\
\hline 6 & 5.113766000 & -1.293223000 & 0.000020000 & 6 & 4.519494000 & -0.896096000 & 0.000202000 \\
\hline 6 & 5.416274000 & 0.076509000 & 0.000013000 & 6 & -1.002423000 & -0.243450000 & -0.000209000 \\
\hline 6 & 4.394288000 & 1.010236000 & -0.000004000 & 6 & -0.324385000 & 0.981533000 & -0.000217000 \\
\hline 6 & -1.743267000 & 2.427071000 & 0.000012000 & 6 & -2.389653000 & -0.342675000 & -0.000066000 \\
\hline 6 & -3.058081000 & 1.980385000 & 0.000012000 & 6 & -3.094779000 & 0.927002000 & 0.000036000 \\
\hline 6 & -3.399730000 & 0.599983000 & 0.000009000 & 6 & -2.381877000 & 2.146930000 & 0.000010000 \\
\hline 6 & -2.371510000 & -0.415205000 & 0.000008000 & 6 & -0.984779000 & 2.200974000 & -0.000112000 \\
\hline 6 & -4.756194000 & 0.175100000 & 0.000008000 & 6 & -3.136740000 & -1.557202000 & 024000 \\
\hline 6 & -5.081650000 & -1.159363000 & -0.000003000 & 6 & -4.505590000 & -1.530248000 & 0.000113000 \\
\hline 6 & -4.065853000 & -2.148833000 & -0.000010000 & 6 & -5.204409000 & -0.291077000 & 0.000211000 \\
\hline 6 & -2.741829000 & -1.786812000 & -0.000004000 & 6 & -4.519495000 & 0.896092000 & 0.000174000 \\
\hline 1 & 1.143701000 & -2.296268000 & 0.000017000 & 1 & 0.467542000 & -3.152943000 & -0.000061000 \\
\hline 1 & 2.234686000 & 2.659637000 & 0.000016000 & 1 & 2.951202000 & -3.070480000 & 0.000142000 \\
\hline 1 & 3.559594000 & -2.768946000 & 0.000024000 & 1 & 2.603186000 & 2.501076000 & -0.000149000 \\
\hline 1 & 5.913282000 & -2.025360000 & 0.000031000 & 1 & 5.067404000 & 2.4575 & 161000 \\
\hline 1 & 6.449808000 & 0.403793000 & 0.000015000 & 1 & 6.288498000 & 0.292090000 & 0.000373000 \\
\hline 1 & 4.628666000 & 2.069925000 & -0.000014000 & 1 & 5.058701000 & -1.837891000 & 0.000292000 \\
\hline 1 & -1.513566000 & 3.485885000 & 0.000009000 & 1 & -2.951219000 & 3.070475000 & 0.000089000 \\
\hline 1 & -3.868767000 & 2.701519000 & -0.000011000 & 1 & -0.467542000 & 3.152946000 & -0.000107000 \\
\hline 1 & -5.537695000 & 0.927878000 & 0.000021000 & 1 & -2.603177000 & -2.501074000 & -0.000102000 \\
\hline 1 & -6.122115000 & -1.463771000 & 0.000000000 & 1 & -5.067400000 & -2.457 & 145000 \\
\hline 1 & -4.342740000 & -3.197039000 & -0.000020000 & 1 & -6.288497000 & -0.292101000 & 0.000317000 \\
\hline 1 & -1.963055000 & -2.540984000 & -0.000006000 & 1 & -5.058694000 & 1.837891000 & 0.000251000 \\
\hline \multicolumn{4}{|c|}{$E=-769.502852500$} & \multicolumn{4}{|c|}{$E=-769.489580999$} \\
\hline \multicolumn{4}{|c|}{ NC6 } & \multicolumn{4}{|c|}{ NC7 } \\
\hline 6 & -0.758046000 & 1.769159000 & 0.000133000 & 6 & 6.266182000 & -0.719340000 & 0.000016000 \\
\hline 6 & -1.917435000 & 2.527329000 & 0.000322000 & 6 & 6.266182000 & 0.719340000 & 0.000064000 \\
\hline 6 & -0.761417000 & 0.368896000 & 0.000056000 & 6 & 5.025385000 & -1.452492000 & -0.000055000 \\
\hline 6 & -1.937491000 & -0.379641000 & -0.000085000 & 6 & 3.882101000 & -0.722706000 & -0.000074000 \\
\hline 6 & -3.162144000 & 0.402528000 & 0.000179000 & 6 & 3.882101000 & 0.722706000 & -0.000030000 \\
\hline 6 & -3.119387000 & 1.814404000 & 0.000389000 & 6 & 2.388574000 & -0.718073000 & -0.000105000 \\
\hline 6 & -2.026189000 & -1.802459000 & -0.000371000 & 6 & 2.388574000 & 0.718073000 & -0.000095000 \\
\hline 6 & -3.244433000 & -2.427384000 & -0.000434000 & 6 & 1.237574000 & -1.445878000 & -0.000084000 \\
\hline 6 & -4.446693000 & -1.668104000 & -0.000155000 & 6 & 0.000000000 & -0.723300000 & -0.000084000 \\
\hline 6 & -4.403042000 & -0.298718000 & 0.000150000 & 6 & 0.000000000 & 0.723300000 & -0.000072000 \\
\hline 6 & 0.758047000 & 1.769157000 & -0.000453000 & 6 & -1.237574000 & -1.445878000 & -0.000103000 \\
\hline 6 & 0.761417000 & 0.368897000 & -0.000523000 & 6 & -2.388574000 & -0.718073000 & -0.000060000 \\
\hline 6 & 1.917437000 & 2.527329000 & -0.000225000 & 6 & -2.388573000 & 0.718073000 & -0.000053000 \\
\hline 6 & 3.119387000 & 1.814405000 & -0.000095000 & 6 & -3.882101000 & -0.722706000 & -0.000137000 \\
\hline 6 & 3.162144000 & 0.402527000 & -0.000035000 & 6 & -3.882101000 & 0.722706000 & -0.000189000 \\
\hline 6 & 1.937492000 & -0.379642000 & -0.000131000 & 6 & -5.025385000 & -1.452492000 & 0.000029000 \\
\hline
\end{tabular}




\begin{tabular}{|c|c|c|c|c|c|c|c|}
\hline 6 & 4.403041000 & -0.298718000 & 0.000223000 & 6 & -6.266182000 & -0.719340000 & 0.000054000 \\
\hline 6 & 4.446692000 & -1.668105000 & 0.000429000 & 6 & -6.266182000 & 0.719340000 & -0.000006000 \\
\hline 6 & 3.244433000 & -2.427384000 & 0.000365000 & 6 & -7.506992000 & -1.394540000 & 0.000160000 \\
\hline 6 & 2.026188000 & -1.802459000 & 0.000063000 & 6 & 7.506992000 & -1.394540000 & 0.000044000 \\
\hline 1 & -1.912490000 & 3.610820000 & 0.000374000 & 6 & 5.025385000 & 1.452492000 & 0.000041000 \\
\hline 1 & -4.060897000 & 2.353443000 & 0.000564000 & 6 & 7.506992000 & 1.394540000 & 0.000131000 \\
\hline 1 & -1.113353000 & -2.385756000 & -0.000582000 & 6 & 1.237574000 & 1.445878000 & -0.000073000 \\
\hline 1 & -3.298577000 & -3.510276000 & -0.000684000 & 6 & -1.237574000 & 1.445878000 & -0.000075000 \\
\hline 1 & -5.401401000 & -2.181668000 & -0.000187000 & 6 & -5.025385000 & 1.452492000 & -0.000091000 \\
\hline 1 & -5.322743000 & 0.277386000 & 0.000366000 & 6 & -7.506992000 & 1.394540000 & 0.000056000 \\
\hline 1 & 1.912490000 & 3.610820000 & -0.000121000 & 6 & -8.702227000 & 0.703253000 & 0.000163000 \\
\hline 1 & 4.060899000 & 2.353442000 & 0.000023000 & 6 & -8.702228000 & -0.703253000 & 0.000215000 \\
\hline 1 & 5.322743000 & 0.277385000 & 0.000264000 & 6 & 8.702227000 & -0.703253000 & 0.000110000 \\
\hline 1 & 5.401400000 & -2.181669000 & 0.000642000 & 6 & 8.702228000 & 0.703252000 & 0.000154000 \\
\hline 1 & 3.298574000 & -3.510276000 & 0.000538000 & 1 & 5.046026000 & -2.536781000 & -0.000081000 \\
\hline 1 & 1.113353000 & -2.385757000 & -0.000007000 & 1 & 5.046026000 & 2.536781000 & 0.000070000 \\
\hline & & & & 1 & 1.218959000 & 2.530211000 & -0.000048000 \\
\hline & -769.488849356 & & & 1 & 1.218959000 & -2.530211000 & -0.000087000 \\
\hline & & & & 1 & -1.218959000 & 2.530211000 & -0.000107000 \\
\hline & & & & 1 & -1.218959000 & -2.530211000 & -0.000125000 \\
\hline & & & & 1 & -5.046026000 & 2.536781000 & -0.000070000 \\
\hline & & & & 1 & -5.046026000 & -2.536781000 & 0.000108000 \\
\hline & & & & 1 & -7.507405000 & 2.479750000 & 0.000014000 \\
\hline & & & & 1 & -9.641767000 & 1.244074000 & 0.000207000 \\
\hline & & & & 1 & -9.641767000 & -1.244074000 & 0.000301000 \\
\hline & & & & 1 & -7.507405000 & -2.479750000 & 0.000201000 \\
\hline & & & & 1 & 7.507405000 & -2.479750000 & 0.000009000 \\
\hline & & & & 1 & 9.641767000 & -1.244074000 & 0.000128000 \\
\hline & & & & 1 & 9.641767000 & 1.244074000 & 0.000206000 \\
\hline & & & & 1 & 7.507405000 & 2.479750000 & 0.000165000 \\
\hline & & & & & 116200807010 & & \\
\hline & & & & $\overline{\mathrm{N}}$ & & & \\
\hline 6 & 3.421773000 & -3.886615000 & 0.000000000 & 6 & -6.007445000 & 0.933877000 & -0.000002000 \\
\hline 6 & 2.741730000 & -5.151484000 & 0.000000000 & 6 & -6.144825000 & -0.497652000 & 0.000046000 \\
\hline 6 & 3.512172000 & -6.331676000 & 0.000000000 & 6 & -4.700858000 & 1.545401000 & -0.000042000 \\
\hline 6 & 1.295310000 & -5.207908000 & 0.000000000 & 6 & -3.633385000 & 0.709962000 & -0.000033000 \\
\hline 6 & 0.637912000 & -4.026644000 & 0.000000000 & 6 & -3.771606000 & -0.729342000 & 0.000013000 \\
\hline 6 & 1.322934000 & -2.750304000 & 0.000000000 & 6 & -2.146149000 & 0.559235000 & -0.000053000 \\
\hline 6 & -0.672495000 & -3.301615000 & 0.000000000 & 6 & -2.283088000 & -0.864581000 & -0.000009000 \\
\hline 6 & -0.007199000 & -2.061019000 & 0.000000000 & 6 & -0.926149000 & 1.172922000 & -0.000090000 \\
\hline 6 & -0.667935000 & -0.835075000 & 0.000000000 & 6 & 0.231131000 & 0.336095000 & -0.000084000 \\
\hline 6 & -2.120859000 & -0.928028000 & 0.000000000 & 6 & 0.092907000 & -1.098998000 & -0.000043000 \\
\hline 6 & -2.056216000 & -3.400155000 & 0.000000000 & 6 & 1.542280000 & 0.939603000 & -0.000117000 \\
\hline 6 & 0.000000000 & 0.440407000 & 0.000000000 & 6 & 2.611421000 & 0.106839000 & -0.000119000 \\
\hline 6 & -0.795860000 & 1.538118000 & 0.000000000 & 6 & 2.472119000 & -1.327337000 & -0.000079000 \\
\hline 6 & -2.242218000 & 1.456296000 & 0.000000000 & 6 & 4.103044000 & -0.059155000 & -0.000107000 \\
\hline 6 & -0.882556000 & 3.026862000 & 0.000000000 & 6 & 3.968861000 & -1.453305000 & -0.000065000 \\
\hline 6 & -2.324195000 & 2.945014000 & 0.000000000 & 6 & 5.345562000 & 0.584176000 & -0.000030000 \\
\hline 6 & -0.217886000 & 4.211088000 & 0.000000000 & 6 & 6.490102000 & -0.300209000 & 0.000077000 \\
\hline
\end{tabular}




\begin{tabular}{|c|c|c|c|c|c|c|c|}
\hline 6 & -1.018641000 & 5.406015000 & 0.000000000 & 6 & 6.317907000 & -1.710140000 & 0.000116000 \\
\hline 6 & -2.456000000 & 5.324921000 & 0.000000000 & 6 & -7.177555000 & 1.724086000 & -0.000006000 \\
\hline 6 & -0.414102000 & 6.684225000 & 0.000000000 & 6 & -4.978505000 & -1.346501000 & 0.000052000 \\
\hline 6 & 4.831184000 & -3.878200000 & 0.000000000 & 6 & -7.443869000 & -1.050998000 & 0.000085000 \\
\hline 6 & 2.671701000 & -2.648653000 & 0.000000000 & 6 & -1.202462000 & -1.699709000 & -0.000004000 \\
\hline 6 & -2.755649000 & -2.191667000 & 0.000000000 & 6 & 1.265302000 & -1.941392000 & -0.000036000 \\
\hline 6 & -2.911679000 & 0.276111000 & 0.000000000 & 6 & 5.065349000 & -2.313563000 & 0.000054000 \\
\hline 6 & -3.117576000 & 4.047639000 & 0.000000000 & 6 & -8.434248000 & 1.150371000 & 0.000034000 \\
\hline 6 & -3.200117000 & 6.527326000 & 0.000000000 & 6 & -8.568535000 & -0.248865000 & 0.000080000 \\
\hline 6 & 4.895207000 & -6.290489000 & 0.000000000 & 1 & -4.617939000 & 2.626748000 & -0.000076000 \\
\hline 6 & 5.559693000 & -5.054508000 & 0.000000000 & 1 & -5.102627000 & -2.423900000 & 0.000087000 \\
\hline 6 & -1.171398000 & 7.837232000 & 0.000000000 & 1 & -1.287923000 & -2.780903000 & 0.000028000 \\
\hline 6 & -2.576989000 & 7.758017000 & 0.000000000 & 1 & -0.804062000 & 2.250619000 & -0.000123000 \\
\hline 1 & 2.998879000 & -7.287910000 & 0.000000000 & 1 & 1.142186000 & -3.018876000 & 0.000004000 \\
\hline 1 & 5.465216000 & -7.212677000 & 0.000000000 & 1 & 1.624399000 & 2.020903000 & -0.000139000 \\
\hline 1 & 6.643311000 & -5.021256000 & 0.000000000 & 1 & 4.966340000 & -3.392423000 & 0.000093000 \\
\hline 1 & 5.345799000 & -2.922606000 & 0.000000000 & 1 & -7.074540000 & 2.804474000 & -0.000041000 \\
\hline 1 & 3.206487000 & -1.705179000 & 0.000000000 & 1 & -9.317551000 & 1.778999000 & 0.000030000 \\
\hline 1 & 0.800001000 & -6.172723000 & 0.000000000 & 1 & -9.555299000 & -0.697917000 & 0.000111000 \\
\hline 1 & -3.840601000 & -2.208823000 & 0.000000000 & 1 & -7.548032000 & -2.131274000 & 0.000120000 \\
\hline 1 & -2.582326000 & -4.347194000 & 0.000000000 & 6 & 5.548312000 & 1.990979000 & -0.000050000 \\
\hline 1 & 1.082855000 & 0.479852000 & 0.000000000 & 6 & 7.785430000 & 0.286638000 & 0.000153000 \\
\hline 1 & -3.993756000 & 0.203306000 & 0.000000000 & 6 & 7.946947000 & 1.650092000 & 0.000131000 \\
\hline 1 & 0.863569000 & 4.292024000 & 0.000000000 & 6 & 6.818518000 & 2.509835000 & 0.000031000 \\
\hline 1 & -4.201292000 & 4.007022000 & 0.000000000 & 1 & 8.652487000 & -0.365895000 & 0.000230000 \\
\hline 1 & 0.669311000 & 6.745800000 & 0.000000000 & 1 & 8.942986000 & 2.078021000 & 0.000191000 \\
\hline 1 & -0.685023000 & 8.806066000 & 0.000000000 & 1 & 6.966632000 & 3.583 & 16000 \\
\hline 1 & -3.169118000 & 8.666118000 & 0.000000000 & 1 & 4.684638000 & 2.646300000 & -0.000131000 \\
\hline 1 & -4.283583000 & 6.466801000 & 0.000000000 & 1 & 7.209243000 & -2.328918000 & 0.000202000 \\
\hline \multicolumn{4}{|c|}{$E=-1153.02429644$} & \multicolumn{4}{|c|}{$E=-1153.02052183$} \\
\hline \multicolumn{4}{|c|}{$\mathrm{NC10}$} & \multicolumn{4}{|c|}{$\mathrm{NC} 11$} \\
\hline 6 & -0.221470000 & -2.598114000 & 0.000028000 & 6 & -3.032367000 & -0.012056000 & -0.000549000 \\
\hline 6 & 0.713839000 & -3.651295000 & 0.000035000 & 6 & -3.725440000 & 1.259644000 & -0.000455000 \\
\hline 6 & 0.227287000 & -1.215372000 & 0.000031000 & 6 & -2.403574000 & 1.961333000 & -0.000652000 \\
\hline 6 & 1.605954000 & -1.052868000 & 0.000042000 & 6 & -1.726441000 & 0.721215000 & -0.000996000 \\
\hline 6 & 2.518636000 & -2.131323000 & 0.000052000 & 6 & -3.686461000 & -1.196780000 & -0.000237000 \\
\hline 6 & 2.737359000 & -0.071830000 & 0.000040000 & 6 & -5.132085000 & -1.146791000 & 0.000152000 \\
\hline 6 & 3.672478000 & -1.177548000 & 0.000065000 & 6 & -5.818545000 & 0.115037000 & 0.000212000 \\
\hline 6 & 3.138765000 & 1.220532000 & -0.000014000 & 6 & -5.074675000 & 1.355498000 & -0.000109000 \\
\hline 6 & 4.564675000 & 1.463331000 & -0.000025000 & 6 & -5.897243000 & -2.331087000 & 0.000453000 \\
\hline 6 & 5.491675000 & 0.365853000 & 0.000001000 & 6 & -7.279988000 & -2.296686000 & 0.000836000 \\
\hline 6 & 5.074969000 & 2.777679000 & -0.000067000 & 6 & -7.950833000 & -1.063538000 & 0.000918000 \\
\hline 6 & 2.100910000 & -3.449149000 & 0.000048000 & 6 & -7.228496000 & 0.116063000 & 0.000622000 \\
\hline 6 & 5.013376000 & -0.999220000 & 0.000040000 & 6 & -1.729301000 & 3.168374000 & 0.000009000 \\
\hline 6 & 6.872868000 & 0.649575000 & -0.000018000 & 6 & -0.330280000 & 3.087470000 & 0.000120000 \\
\hline 6 & 6.436200000 & 3.023096000 & -0.000084000 & 6 & 0.374229000 & 1.867453000 & -0.000249000 \\
\hline 6 & 7.342112000 & 1.950735000 & -0.000059000 & 6 & -0.343419000 & 0.603279000 & -0.000750000 \\
\hline 1 & 2.784462000 & -4.289665000 & 0.000057000 & 6 & 1.823400000 & 1.866827000 & 0.000002000 \\
\hline 1 & 2.457517000 & 2.064375000 & -0.000042000 & 6 & 2.439020000 & 0.663659000 & -0.000217000 \\
\hline
\end{tabular}




\begin{tabular}{|c|c|c|c|c|c|c|c|}
\hline 1 & 5.728925000 & -1.814221000 & 0.000045000 & 6 & 1.710991000 & -0.594790000 & -0.000660000 \\
\hline 1 & 4.374916000 & 3.607109000 & -0.000086000 & 6 & 0.362954000 & -0.661162000 & -0.000914000 \\
\hline 1 & 6.803429000 & 4.043152000 & -0.000117000 & 6 & 3.719000000 & -0.108905000 & -0.000159000 \\
\hline 1 & 8.409199000 & 2.142335000 & -0.000073000 & 6 & 3.018626000 & -1.322390000 & -0.000593000 \\
\hline 1 & 7.573812000 & -0.179083000 & 0.000001000 & 6 & 5.118994000 & -0.040184000 & 0.000215000 \\
\hline 6 & -5.732513000 & 0.743850000 & -0.000018000 & 6 & 5.791659000 & -1.318737000 & 0.000105000 \\
\hline 6 & -6.147499000 & -0.617202000 & -0.000020000 & 6 & 5.048290000 & -2.532087000 & -0.000340000 \\
\hline 6 & -5.250236000 & -1.676016000 & -0.000002000 & 6 & 3.660704000 & -2.561774000 & -0.000689000 \\
\hline 6 & -3.896182000 & -1.335445000 & 0.000028000 & 6 & 5.886209000 & 1.154624000 & 0.000673000 \\
\hline 6 & -3.453347000 & -0.006233000 & 0.000033000 & 6 & 7.258191000 & 1.099464000 & 0.001007000 \\
\hline 6 & -2.484598000 & -1.832408000 & -0.000001000 & 6 & 7.926661000 & -0.150257000 & 0.000900000 \\
\hline 6 & -2.026380000 & -0.452716000 & 0.000000000 & 6 & 7.212565000 & -1.324074000 & 0.000463000 \\
\hline 6 & -1.641941000 & -2.887621000 & 0.000022000 & 1 & -3.189334000 & -2.160642000 & -0.000329000 \\
\hline 6 & -4.329294000 & 1.087730000 & 0.000004000 & 1 & -5.610774000 & 2.298296000 & 0.000047000 \\
\hline 1 & -5.597915000 & -2.702074000 & -0.000009000 & 1 & -5.379220000 & -3.284830000 & 0.000388000 \\
\hline 1 & -1.973784000 & -3.920147000 & 0.000032000 & 1 & -7.845569000 & -3.221623000 & 0.001071000 \\
\hline 6 & -6.678977000 & 1.803628000 & -0.000044000 & 1 & -9.034627000 & -1.035882000 & 0.001216000 \\
\hline 6 & -6.274198000 & 3.116539000 & -0.000051000 & 1 & -7.747694000 & 1.069163000 & 0.000694000 \\
\hline 1 & -7.736033000 & 1.558528000 & -0.000059000 & 1 & -2.230070000 & 4.129072000 & 16000 \\
\hline 1 & -7.011454000 & 3.911270000 & -0.000071000 & 1 & 0.250148000 & 4.004448000 & 0.000528000 \\
\hline 1 & -4.594764000 & 4.486204000 & -0.000039000 & 1 & 2.352967000 & 2.813178000 & 0.000371000 \\
\hline 6 & -3.943930000 & 2.454428000 & -0.000004000 & 1 & -0.197897000 & -1.588321000 & -0.001257000 \\
\hline 6 & -4.895281000 & 3.444508000 & -0.000032000 & 1 & 5.603422000 & -3.464355000 & -0.000398000 \\
\hline 1 & -2.888833000 & 2.703455000 & 0.000013000 & 1 & 3.122334000 & -3.501918000 & -0.001016000 \\
\hline 1 & -7.213530000 & -0.819653000 & -0.000041000 & 1 & 5.372738000 & 2.109402000 & 0.000753000 \\
\hline 6 & -0.717677000 & -0.118238000 & 0.000026000 & 1 & 7.838602000 & 2.015164000 & 7000 \\
\hline 1 & -0.351327000 & 0.901448000 & 0.000040000 & 1 & 9.010442000 & -0.175407000 & 0.001166000 \\
\hline 1 & 0.329917000 & -4.666358000 & 0.000033000 & 1 & 7.730468000 & -2.277608000 & 0.000384000 \\
\hline \multicolumn{4}{|c|}{$E=-1153.01873791$} & \multicolumn{4}{|c|}{$E=-1153.01867248$} \\
\hline \multicolumn{4}{|c|}{$\mathrm{NC} 12$} & \multicolumn{4}{|c|}{$\mathrm{NC} 13$} \\
\hline 6 & 3.371265000 & 4.575387000 & 0.000000000 & 6 & 0.000000000 & 5.794014000 & -1.669609000 \\
\hline 6 & 4.577356000 & 3.796378000 & 0.000000000 & 6 & 0.000000000 & 5.858503000 & -3.052630000 \\
\hline 6 & 2.075825000 & 3.926020000 & 0.000000000 & 6 & 0.000000000 & 4.677375000 & -3.808666000 \\
\hline 6 & 2.070434000 & 2.574581000 & 0.000000000 & 6 & 0.000000000 & 3.448291000 & -3.171392000 \\
\hline 6 & 3.288102000 & 1.788767000 & 0.000000000 & 6 & 0.000000000 & 3.350009000 & -1.766317000 \\
\hline 6 & 1.279460000 & 1.302025000 & 0.000000000 & 6 & 0.000000000 & 4.558931000 & -0.992838000 \\
\hline 6 & 2.456385000 & 0.542585000 & 0.000000000 & 6 & 0.000000000 & 2.057096000 & -1.112258000 \\
\hline 6 & 0.000362000 & 0.730332000 & 0.000000000 & 6 & 0.000000000 & 2.053480000 & 0.239107000 \\
\hline 6 & -0.000362000 & -0.730332000 & 0.000000000 & 6 & 0.000000000 & 3.275354000 & 1.017581000 \\
\hline 6 & -1.279460000 & -1.302025000 & 0.000000000 & 6 & 0.000000000 & 4.503085000 & 0.454961000 \\
\hline 6 & -2.456385000 & -0.542585000 & 0.000000000 & 6 & 0.000000000 & 1.269229000 & 1.517591000 \\
\hline 6 & -2.070434000 & -2.574581000 & 0.000000000 & 6 & 0.000000000 & 2.451284000 & 2.267869000 \\
\hline 6 & -3.288102000 & -1.788767000 & 0.000000000 & 6 & 0.000000000 & 0.000000000 & 2.116345000 \\
\hline 6 & 1.213944000 & -1.471474000 & 0.000000000 & 6 & 0.000000000 & 0.000000000 & 3.578805000 \\
\hline 6 & -1.213944000 & 1.471474000 & 0.000000000 & 6 & 0.000000000 & 1.230999000 & 4.292330000 \\
\hline 6 & 3.474773000 & 5.980012000 & 0.000000000 & 6 & 0.000000000 & 2.467465000 & 3.664127000 \\
\hline 6 & -2.075825000 & -3.926020000 & 0.000000000 & 6 & 0.000000000 & -1.269229000 & 1.517591000 \\
\hline 6 & -3.371265000 & -4.575387000 & 0.000000000 & 6 & 0.000000000 & -2.451284000 & 2.267869000 \\
\hline 6 & -4.577356000 & -3.796378000 & 0.000000000 & 6 & 0.000000000 & -2.467465000 & 3.664127000 \\
\hline
\end{tabular}




\begin{tabular}{|c|c|c|c|c|c|c|c|}
\hline 6 & -3.474773000 & -5.980012000 & 0.000000000 & 6 & 0.000000000 & -1.230999000 & 4.292330000 \\
\hline 6 & 4.517663000 & 2.348405000 & 0.000000000 & 6 & 0.000000000 & -2.053480000 & 0.239107000 \\
\hline 6 & 5.814764000 & 4.469115000 & 0.000000000 & 6 & 0.000000000 & -3.275354000 & 1.017581000 \\
\hline 6 & 2.456385000 & -0.855162000 & 0.000000000 & 6 & 0.000000000 & -2.057096000 & -1.112258000 \\
\hline 6 & -2.456385000 & 0.855162000 & 0.000000000 & 6 & 0.000000000 & -3.350009000 & -1.766317000 \\
\hline 6 & -4.517663000 & -2.348405000 & 0.000000000 & 6 & 0.000000000 & -4.558931000 & -0.992838000 \\
\hline 6 & -5.814764000 & -4.469115000 & 0.000000000 & 6 & 0.000000000 & -4.503085000 & 0.454961000 \\
\hline 6 & 4.706173000 & 6.612714000 & 0.000000000 & 6 & 0.000000000 & -3.448291000 & -3.171392000 \\
\hline 6 & 5.884318000 & 5.851895000 & 0.000000000 & 6 & 0.000000000 & -4.677375000 & -3.808666000 \\
\hline 6 & -4.706173000 & -6.612714000 & 0.000000000 & 6 & 0.000000000 & -5.858503000 & -3.052630000 \\
\hline 6 & -5.884318000 & -5.851895000 & 0.000000000 & 6 & 0.000000000 & -5.794014000 & -1.669609000 \\
\hline 1 & 1.178243000 & 4.534581000 & 0.000000000 & 1 & 0.000000000 & 6.708334000 & -1.084830000 \\
\hline 1 & 5.439880000 & 1.777777000 & 0.000000000 & 1 & 0.000000000 & 6.821474000 & -3.550565000 \\
\hline 1 & 3.368499000 & -1.439783000 & 0.000000000 & 1 & 0.000000000 & 4.726392000 & -4.891659000 \\
\hline 1 & 1.148770000 & -2.553607000 & 0.000000000 & 1 & 0.000000000 & 2.534879000 & -3.757546000 \\
\hline 1 & 2.563375000 & 6.569310000 & 0.000000000 & 1 & 0.000000000 & 1.159261000 & -1.718936000 \\
\hline 1 & 4.759383000 & 7.695468000 & 0.000000000 & 1 & 0.000000000 & 5.426459000 & 1.023790000 \\
\hline 1 & 6.849261000 & 6.345987000 & 0.000000000 & 1 & 0.000000000 & 1.183911000 & 5.376290000 \\
\hline 1 & 6.726898000 & 3.880988000 & 0.000000000 & 1 & 0.000000000 & 3.385684000 & 4.238894000 \\
\hline 1 & -3.368499000 & 1.439783000 & 0.000000000 & 1 & 0.000000000 & -3.385684000 & 4.238894000 \\
\hline 1 & -1.148770000 & 2.553607000 & 0.000000000 & 1 & 0.000000000 & -1.183911000 & 5.376290000 \\
\hline 1 & -1.178243000 & -4.534581000 & 0.000000000 & 1 & 0.000000000 & -1.159261000 & -1.718936000 \\
\hline 1 & -5.439880000 & -1.777777000 & 0.000000000 & 1 & 0.000000000 & -5.426459000 & 1.023790000 \\
\hline 1 & -2.563375000 & -6.569310000 & 0.000000000 & 1 & 0.000000000 & -2.534879000 & -3.757546000 \\
\hline 1 & -4.759383000 & -7.695468000 & 0.000000000 & 1 & 0.000000000 & -4.726392000 & -4.891659000 \\
\hline 1 & -6.849261000 & -6.345987000 & 0.000000000 & 1 & 0.000000000 & -6.821474000 & -3.550565000 \\
\hline 1 & -6.726898000 & -3.880988000 & 0.000000000 & 1 & 0.000000000 & -6.708334000 & -1.084830000 \\
\hline \multicolumn{4}{|c|}{$E=-1153.01717338$} & \multicolumn{4}{|c|}{$E=-1153.01561059$} \\
\hline \multicolumn{4}{|c|}{$\mathrm{NC} 14$} & \multicolumn{4}{|c|}{$\mathrm{NC} 15$} \\
\hline 6 & 2.640548000 & 2.888463000 & 0.000000000 & 6 & & & 0.000118000 \\
\hline 6 & 3.798891000 & 3.661896000 & 0.000000000 & 6 & 6.298370000 & 0.636473000 & 0.000079000 \\
\hline 6 & 5.038481000 & 2.908248000 & 0.000000000 & 6 & 4.654235000 & 2.474307000 & 0.000056000 \\
\hline 6 & 5.022706000 & 1.493788000 & 0.000000000 & 6 & 3.662874000 & 1.496405000 & -0.000069000 \\
\hline 6 & 3.837275000 & 0.755637000 & 0.000000000 & 6 & 3.953865000 & 0.126526000 & -0.000106000 \\
\hline 6 & 2.657974000 & 1.488294000 & 0.000000000 & 6 & 2.187687000 & 1.198632000 & -0.000071000 \\
\hline 6 & 3.845850000 & 5.085845000 & 0.000000000 & 6 & 2.487307000 & -0.205007000 & -0.000093000 \\
\hline 6 & 5.049030000 & 5.740759000 & 0.000000000 & 6 & 0.915227000 & 1.670574000 & -0.000040000 \\
\hline 6 & 6.268705000 & 5.010269000 & 0.000000000 & 6 & -0.149353000 & 0.701846000 & -0.000035000 \\
\hline 6 & 6.261040000 & 3.639133000 & 0.000000000 & 6 & 0.149353000 & -0.701846000 & -0.000052000 \\
\hline 6 & 1.142684000 & 1.468610000 & 0.000000000 & 6 & -1.515262000 & 1.153933000 & -0.000013000 \\
\hline 6 & 1.127476000 & 2.877734000 & 0.000000000 & 6 & -2.487307000 & 0.205007000 & -0.000010000 \\
\hline 6 & 0.000000000 & 0.689630000 & 0.000000000 & 6 & -2.187687000 & -1.198632000 & -0.000024000 \\
\hline 6 & -1.254304000 & 1.446041000 & 0.000000000 & 6 & -3.953865000 & -0.126526000 & 0.000000000 \\
\hline 6 & -1.234212000 & 2.850890000 & 0.000000000 & 6 & -3.662874000 & -1.496405000 & -0.000010000 \\
\hline 6 & -0.045541000 & 3.602296000 & 0.000000000 & 6 & -5.259994000 & 0.371758000 & 0.000007000 \\
\hline 6 & 0.001711000 & -0.754749000 & 0.000000000 & 6 & -6.298370000 & -0.636473000 & 0.000006000 \\
\hline 6 & -1.208956000 & -1.359092000 & 0.000000000 & 6 & -5.968530000 & -2.016761000 & -0.000002000 \\
\hline 6 & -2.459064000 & -0.616972000 & 0.000000000 & 6 & 5.259994000 & -0.371758000 & -0.000024000 \\
\hline 6 & -2.511152000 & 0.736738000 & 0.000000000 & 6 & 1.515262000 & -1.153933000 & -0.000079000 \\
\hline
\end{tabular}




\begin{tabular}{|c|c|c|c|c|c|c|c|}
\hline 6 & -1.974081000 & -2.637753000 & 0.000000000 & 6 & -0.915227000 & -1.670574000 & -0.000046000 \\
\hline 6 & -3.215392000 & -1.899912000 & 0.000000000 & 6 & -4.654234000 & -2.474306000 & -0.000011000 \\
\hline 6 & -1.931074000 & -3.995871000 & 0.000000000 & 1 & 4.433903000 & 3.535114000 & 0.000094000 \\
\hline 6 & -3.191817000 & -4.686370000 & 0.000000000 & 1 & 1.719208000 & -2.219103000 & -0.000090000 \\
\hline 6 & -4.430044000 & -3.951010000 & 0.000000000 & 1 & 0.670801000 & 2.727261000 & -0.000016000 \\
\hline 6 & -4.428313000 & -2.513793000 & 0.000000000 & 1 & -0.670801000 & -2.727261000 & -0.000059000 \\
\hline 6 & -3.245098000 & -6.099752000 & 0.000000000 & 1 & -1.719208000 & 2.219103000 & -0.000002000 \\
\hline 6 & -4.448389000 & -6.773475000 & 0.000000000 & 1 & -4.433904000 & -3.535114000 & -0.000019000 \\
\hline 6 & -5.659460000 & -6.054501000 & 0.000000000 & 6 & -5.620343000 & 1.747416000 & 0.000014000 \\
\hline 6 & -5.645058000 & -4.675573000 & 0.000000000 & 6 & -7.651767000 & -0.198496000 & 0.000012000 \\
\hline 1 & 5.974864000 & 0.973677000 & 0.000000000 & 6 & -7.965496000 & 1.137844000 & 0.000017000 \\
\hline 1 & 3.859528000 & -0.327581000 & 0.000000000 & 6 & -6.940498000 & 2.119707000 & 0.000019000 \\
\hline 1 & 2.914819000 & 5.641489000 & 0.000000000 & 1 & -8.439904000 & -0.944472000 & 0.000011000 \\
\hline 1 & 5.077862000 & 6.824604000 & 0.000000000 & 1 & -9.003319000 & 1.451284000 & 0.000021000 \\
\hline 1 & 7.210448000 & 5.547334000 & 0.000000000 & 1 & -7.208910000 & 3.170191000 & 0.000023000 \\
\hline 1 & 7.195761000 & 3.087743000 & 0.000000000 & 1 & -4.835736000 & 2.495632000 & 0.000015000 \\
\hline 1 & -2.186200000 & 3.371354000 & 0.000000000 & 1 & -6.783708000 & -2.732876000 & -0.000002000 \\
\hline 1 & -0.076837000 & 4.685186000 & 0.000000000 & 6 & 7.651767000 & 0.198496000 & 0.000150000 \\
\hline 1 & 0.943984000 & -1.290125000 & 0.000000000 & 6 & 5.620343000 & -1.747416000 & -0.000040000 \\
\hline 1 & -3.438642000 & 1.299105000 & 0.000000000 & 6 & 7.965496000 & -1.137844000 & 0.000128000 \\
\hline 1 & -1.008736000 & -4.566426000 & 0.000000000 & 6 & 6.940499000 & -2.119706000 & 0.000035000 \\
\hline 1 & -5.371264000 & -1.978066000 & 0.000000000 & 1 & 8.439903000 & 0.944473000 & 0.000223000 \\
\hline 1 & -2.312009000 & -6.653811000 & 0.000000000 & 1 & 9.003319000 & -1.451283000 & 0.000183000 \\
\hline 1 & -4.463733000 & -7.857446000 & 0.000000000 & 1 & 7.208910000 & -3.170191000 & 0.000021000 \\
\hline 1 & -6.603650000 & -6.587245000 & 0.000000000 & 1 & 4.835736000 & -2.495632000 & -0.000117000 \\
\hline 1 & -6.578714000 & -4.122481000 & 0.000000000 & 1 & 6.783709000 & 2.732876000 & 0.000203000 \\
\hline \multicolumn{4}{|c|}{$E=-1153.01234970$} & \multicolumn{4}{|c|}{$E=-1153.01222254$} \\
\hline \multicolumn{4}{|c|}{$\mathrm{NC16}$} & \multicolumn{4}{|c|}{$\mathrm{NC17}$} \\
\hline 6 & 2.640548000 & 2.888463000 & 0.000000000 & 6 & 2.968512000 & 0.821208000 & 0.000085000 \\
\hline 6 & 3.798891000 & 3.661896000 & 0.000000000 & 6 & 3.798128000 & -0.307334000 & 0.000107000 \\
\hline 6 & 5.038481000 & 2.908248000 & 0.000000000 & 6 & 2.587881000 & -1.210924000 & 0.000188000 \\
\hline 6 & 5.022706000 & 1.493788000 & 0.000000000 & 6 & 1.746898000 & -0.072105000 & 0.000177000 \\
\hline 6 & 3.837275000 & 0.755637000 & 0.000000000 & 6 & 3.469692000 & 2.117065000 & -0.000020000 \\
\hline 6 & 2.657974000 & 1.488294000 & 0.000000000 & 6 & 4.859548000 & 2.241714000 & -0.000090000 \\
\hline 6 & 3.845850000 & 5.085845000 & 0.000000000 & 6 & 5.724873000 & 1.121515000 & -0.000059000 \\
\hline 6 & 5.049030000 & 5.740759000 & 0.000000000 & 6 & 5.189679000 & -0.226163000 & 0.000046000 \\
\hline 6 & 6.268705000 & 5.010269000 & 0.000000000 & 6 & 2.088152000 & -2.490797000 & 0.000027000 \\
\hline 6 & 6.261040000 & 3.639133000 & 0.000000000 & 6 & 0.680574000 & -2.602930000 & -0.000072000 \\
\hline 6 & 1.142684000 & 1.468610000 & 0.000000000 & 6 & -0.176260000 & -1.496361000 & -0.000074000 \\
\hline 6 & 1.127476000 & 2.877734000 & 0.000000000 & 6 & 0.370311000 & -0.137839000 & 0.000024000 \\
\hline 6 & 0.000000000 & 0.689630000 & 0.000000000 & 6 & 7.140466000 & 1.275361000 & -0.000131000 \\
\hline 6 & -1.254304000 & 1.446041000 & 0.000000000 & 6 & 7.973299000 & 0.185712000 & -0.000102000 \\
\hline 6 & -1.234212000 & 2.850890000 & 0.000000000 & 6 & 7.440071000 & -1.131707000 & -0.000002000 \\
\hline 6 & -0.045541000 & 3.602296000 & 0.000000000 & 6 & 6.084823000 & -1.333874000 & 0.000068000 \\
\hline 6 & 0.001711000 & -0.754749000 & 0.000000000 & 6 & -1.614782000 & -1.685202000 & -0.000201000 \\
\hline 6 & -1.208956000 & -1.359092000 & 0.000000000 & 6 & -2.383381000 & -0.575382000 & -0.000236000 \\
\hline 6 & -2.459064000 & -0.616972000 & 0.000000000 & 6 & -1.828371000 & 0.774272000 & -0.000167000 \\
\hline 6 & -2.511152000 & 0.736738000 & 0.000000000 & 6 & -0.503752000 & 1.021592000 & -0.000041000 \\
\hline 6 & -1.974081000 & -2.637753000 & 0.000000000 & 6 & -3.751387000 & 0.019257000 & -0.000333000 \\
\hline
\end{tabular}




\begin{tabular}{|c|c|c|c|c|c|c|c|}
\hline 6 & -3.215392000 & -1.899912000 & 0.000000000 & 6 & -3.220299000 & 1.316720000 & -0.000267000 \\
\hline 6 & -1.931074000 & -3.995871000 & 0.000000000 & 6 & -5.130880000 & -0.236565000 & -0.000107000 \\
\hline 6 & -3.191817000 & -4.686370000 & 0.000000000 & 6 & -5.968658000 & 0.939643000 & 0.000140000 \\
\hline 6 & -4.430044000 & -3.951010000 & 0.000000000 & 6 & -5.394606000 & 2.242777000 & 0.000197000 \\
\hline 6 & -4.428313000 & -2.513793000 & 0.000000000 & 6 & -4.024442000 & 2.458811000 & 0.000021000 \\
\hline 6 & -3.245098000 & -6.099752000 & 0.000000000 & 6 & -5.730400000 & -1.523105000 & -0.000113000 \\
\hline 6 & -4.448389000 & -6.773475000 & 0.000000000 & 6 & -7.097769000 & -1.653018000 & 0.000119000 \\
\hline 6 & -5.659460000 & -6.054501000 & 0.000000000 & 6 & -7.927611000 & -0.504704000 & 0.000355000 \\
\hline 6 & -5.645058000 & -4.675573000 & 0.000000000 & 6 & -7.377046000 & 0.754574000 & 0.000362000 \\
\hline 1 & 5.974864000 & 0.973677000 & 0.000000000 & 1 & 2.835168000 & 2.995333000 & -0.000041000 \\
\hline 1 & 3.859528000 & -0.327581000 & 0.000000000 & 1 & 5.306556000 & 3.230349000 & -0.000168000 \\
\hline 1 & 2.914819000 & 5.641489000 & 0.000000000 & 1 & 2.711687000 & -3.376770000 & 0.000002000 \\
\hline 1 & 5.077862000 & 6.824604000 & 0.000000000 & 1 & 0.233766000 & -3.591802000 & -0.000161000 \\
\hline 1 & 7.210448000 & 5.547334000 & 0.000000000 & 1 & 7.553526000 & 2.278903000 & -0.000209000 \\
\hline 1 & 7.195761000 & 3.087743000 & 0.000000000 & 1 & 9.048498000 & 0.324588000 & -0.000157000 \\
\hline 1 & -2.186200000 & 3.371354000 & 0.000000000 & 1 & 8.116089000 & -1.979427000 & 0.000018000 \\
\hline 1 & -0.076837000 & 4.685186000 & 0.000000000 & 1 & 5.676371000 & -2.338203000 & 0.000144000 \\
\hline 1 & 0.943984000 & -1.290125000 & 0.000000000 & 1 & -2.014154000 & -2.693552000 & -0.000253000 \\
\hline 1 & -3.438642000 & 1.299105000 & 0.000000000 & 1 & -0.073036000 & 2.016091000 & 30000 \\
\hline 1 & -1.008736000 & -4.566426000 & 0.000000000 & 1 & -6.070429000 & 3.091687000 & 0.000410000 \\
\hline 1 & -5.371264000 & -1.978066000 & 0.000000000 & 1 & -3.617630000 & 3.462933000 & 0.000097000 \\
\hline 1 & -2.312009000 & -6.653811000 & 0.000000000 & 1 & -5.093141000 & -2.400156000 & -0.000307000 \\
\hline 1 & -4.463733000 & -7.857446000 & 0.000000000 & 1 & -7.549764000 & -2.638457000 & 0.000114000 \\
\hline 1 & -6.603650000 & -6.587245000 & 0.000000000 & 1 & -9.005018000 & -0.624943000 & 0.000529000 \\
\hline 1 & -6.578714000 & -4.122481000 & 0.000000000 & 1 & -8.018056000 & 1.630165000 & 0.000543000 \\
\hline \multicolumn{4}{|c|}{$E=-1153.01234970$} & \multicolumn{4}{|c|}{$E=-1153.00744118$} \\
\hline \multicolumn{4}{|c|}{$\mathrm{NC} 18$} & \multicolumn{4}{|c|}{$\mathrm{NC} 19$} \\
\hline 6 & 5.432295000 & 1.190436000 & & 6 & & & -0.000618000 \\
\hline 6 & 6.217222000 & 0.014212000 & 0.000075000 & 6 & -2.333232000 & 1.784677000 & -0.000370000 \\
\hline 6 & 5.652758000 & -1.261854000 & 0.000047000 & 6 & -3.798299000 & 1.497442000 & 0.000046000 \\
\hline 6 & 4.266943000 & -1.316929000 & -0.000053000 & 6 & -3.554276000 & 0.116903000 & -0.000212000 \\
\hline 6 & 3.456058000 & -0.174870000 & -0.000107000 & 6 & -0.836035000 & -0.174464000 & -0.000510000 \\
\hline 6 & 3.041105000 & -2.199215000 & -0.000025000 & 6 & 0.263697000 & 0.773208000 & -0.000372000 \\
\hline 6 & 2.216083000 & -1.056128000 & -0.000055000 & 6 & 0.017866000 & 2.216842000 & -0.000104000 \\
\hline 6 & 0.831313000 & -1.120374000 & -0.000026000 & 6 & -1.348407000 & 2.706241000 & -0.000005000 \\
\hline 6 & 0.282720000 & -2.480432000 & 0.000045000 & 6 & 1.595339000 & 0.416834000 & -0.000460000 \\
\hline 6 & 2.539911000 & -3.481713000 & 0.000044000 & 6 & 2.658398000 & 1.351671000 & -0.000170000 \\
\hline 6 & -0.044580000 & 0.027938000 & -0.000067000 & 6 & 2.441422000 & 2.708320000 & 0.000190000 \\
\hline 6 & -1.374632000 & -0.220375000 & -0.000032000 & 6 & 1.089536000 & 3.116413000 & 0.000165000 \\
\hline 6 & -1.924805000 & -1.564991000 & 0.000043000 & 6 & -5.089354000 & 2.032064000 & 0.000472000 \\
\hline 6 & -2.755972000 & 0.339344000 & -0.000037000 & 6 & -6.130889000 & 1.116388000 & 0.000630000 \\
\hline 6 & -3.301715000 & -0.997402000 & 0.000037000 & 6 & -5.919365000 & -0.291730000 & 0.000361000 \\
\hline 6 & -3.540635000 & 1.448948000 & -0.000079000 & 6 & -4.582175000 & -0.837247000 & -0.000090000 \\
\hline 6 & -4.962536000 & 1.239594000 & -0.000045000 & 6 & -7.010693000 & -1.200865000 & 0.000507000 \\
\hline 6 & -5.507298000 & -0.093859000 & 0.000030000 & 6 & -6.802650000 & -2.559387000 & 0.000223000 \\
\hline 6 & -5.857635000 & 2.334920000 & -0.000084000 & 6 & -5.487176000 & -3.085720000 & -0.000223000 \\
\hline 6 & 3.981916000 & 1.118517000 & -0.000057000 & 6 & -4.400977000 & -2.245097000 & -0.000371000 \\
\hline 6 & 1.138904000 & -3.593549000 & 0.000077000 & 6 & 2.600045000 & -0.715196000 & -0.000283000 \\
\hline 6 & -1.147743000 & -2.673874000 & 0.000079000 & 6 & 3.649705000 & 0.212206000 & 0.000022000 \\
\hline
\end{tabular}




\begin{tabular}{|c|c|c|c|c|c|c|c|}
\hline 6 & -4.639436000 & -1.239255000 & 0.000071000 & 6 & 2.816222000 & -2.087749000 & -0.000495000 \\
\hline 6 & -6.913460000 & -0.248523000 & 0.000062000 & 6 & 4.148368000 & -2.503470000 & -0.000334000 \\
\hline 6 & -7.223686000 & 2.146565000 & -0.000051000 & 6 & 5.230987000 & -1.591642000 & -0.000021000 \\
\hline 6 & -7.756431000 & 0.842730000 & 0.000023000 & 6 & 4.992554000 & -0.161287000 & 0.000149000 \\
\hline 1 & 6.276772000 & -2.147583000 & 0.000095000 & 6 & 6.582163000 & -2.041067000 & 0.000108000 \\
\hline 1 & 0.687929000 & -4.580341000 & 0.000132000 & 6 & 7.626365000 & -1.151918000 & 0.000368000 \\
\hline 1 & 3.167473000 & -4.364863000 & 0.000077000 & 6 & 7.383471000 & 0.248434000 & 0.000518000 \\
\hline 1 & 0.376348000 & 1.025322000 & -0.000126000 & 6 & 6.101536000 & 0.732319000 & 0.000415000 \\
\hline 1 & -1.545324000 & -3.682969000 & 0.000132000 & 1 & -0.623884000 & -1.237215000 & -0.000558000 \\
\hline 1 & -3.149072000 & 2.460280000 & -0.000135000 & 1 & -1.527253000 & 3.776033000 & 0.000334000 \\
\hline 1 & -5.068267000 & -2.235326000 & 0.000128000 & 1 & 3.238670000 & 3.441846000 & 0.000467000 \\
\hline 1 & -5.447650000 & 3.339717000 & -0.000141000 & 1 & 0.862452000 & 4.177492000 & 0.000384000 \\
\hline 1 & -7.889671000 & 3.001995000 & -0.000082000 & 1 & -5.283006000 & 3.098019000 & 0.000668000 \\
\hline 1 & -8.830920000 & 0.698557000 & 0.000049000 & 1 & -7.156059000 & 1.472059000 & 0.000953000 \\
\hline 1 & -7.324272000 & -1.252920000 & 0.000119000 & 1 & -8.020500000 & -0.803529000 & 0.000848000 \\
\hline 6 & 6.038622000 & 2.479529000 & 0.000070000 & 1 & -7.648625000 & -3.237289000 & 0.000339000 \\
\hline 6 & 5.282085000 & 3.622385000 & 0.000041000 & 1 & -5.342375000 & -4.160181000 & -0.000444000 \\
\hline 1 & 7.122085000 & 2.541433000 & 0.000129000 & 1 & -3.393516000 & -2.645388000 & -0.000710000 \\
\hline 1 & 5.763699000 & 4.593580000 & 0.000078000 & 1 & 2.010549000 & -2.812202000 & -0.000744000 \\
\hline 1 & 3.280071000 & 4.459484000 & -0.000057000 & 1 & 4.376195000 & -3.564287000 & -0.000465000 \\
\hline 6 & 3.231724000 & 2.3299 & -0.000084000 & 1 & 6.773996000 & 4000 & 010000 \\
\hline 6 & 3.863269000 & 3.545435000 & -0.000035000 & 1 & 8.647943000 & -1.514857000 & 0.000458000 \\
\hline 1 & 2.149829000 & 2.278792000 & -0.000146000 & 1 & 8.223262000 & 0.934260000 & 0.000719000 \\
\hline 1 & 7.296780000 & 0.122083000 & 0.000140000 & 1 & 5.914738000 & 1.800324000 & 0.000535000 \\
\hline \multicolumn{4}{|c|}{$E=-1153.01136626$} & \multicolumn{4}{|c|}{$E=-1153.00746539$} \\
\hline \multicolumn{4}{|c|}{$\mathrm{NC} 20$} & \multicolumn{4}{|c|}{$\mathrm{NC} 21$} \\
\hline 6 & 1.651235000 & -0.616001000 & -0.000174000 & 6 & 2.142730000 & 0.795220000 & -0.000118000 \\
\hline 6 & 2.091286000 & -2.006306000 & -0.000196000 & 6 & 1.364042000 & -0.437945000 & -0.000157000 \\
\hline 6 & 3.506119000 & -1.528705000 & -0.000183000 & 6 & 2.641391000 & -1.212346000 & -0.000085000 \\
\hline 6 & 3.082225000 & -0.192330000 & -0.000190000 & 6 & 3.388132000 & -0.025751000 & -0.000039000 \\
\hline 6 & 0.350300000 & -0.261050000 & -0.000076000 & 6 & 1.573014000 & 2.018399000 & -0.000041000 \\
\hline 6 & -0.621605000 & -1.339182000 & -0.000018000 & 6 & 0.122884000 & 2.077869000 & -0.000061000 \\
\hline 6 & -0.185824000 & -2.738422000 & -0.000028000 & 6 & -0.652410000 & 0.834103000 & -0.000133000 \\
\hline 6 & 1.233115000 & -3.046207000 & -0.000099000 & 6 & 0.016802000 & -0.454483000 & -0.000153000 \\
\hline 6 & -1.991920000 & -1.161923000 & 0.000065000 & 6 & -0.523809000 & 3.318235000 & 0.000021000 \\
\hline 6 & -2.913210000 & -2.237207000 & 0.000112000 & 6 & -1.928617000 & 3.454367000 & 0.000041000 \\
\hline 6 & -2.518825000 & -3.551458000 & 0.000087000 & 6 & -2.642271000 & 2.282381000 & -0.000066000 \\
\hline 6 & -1.125028000 & -3.774900000 & 0.000025000 & 6 & -2.022707000 & 1.008841000 & -0.000169000 \\
\hline 6 & 4.856526000 & -1.887872000 & -0.000012000 & 6 & 3.236886000 & -2.475858000 & -0.000086000 \\
\hline 6 & 5.768101000 & -0.842621000 & 0.000137000 & 6 & 4.623806000 & -2.499045000 & -0.000040000 \\
\hline 6 & 5.372667000 & 0.525326000 & 0.000142000 & 6 & 5.413686000 & -1.314360000 & -0.000001000 \\
\hline 6 & 3.975110000 & 0.889234000 & -0.000009000 & 6 & 4.790904000 & -0.011535000 & -0.000005000 \\
\hline 6 & 6.334337000 & 1.570657000 & 0.000313000 & 6 & 6.833038000 & -1.374311000 & 0.000038000 \\
\hline 6 & 5.949115000 & 2.889925000 & 0.000342000 & 6 & 7.591970000 & -0.228613000 & 0.000067000 \\
\hline 6 & 4.575657000 & 3.237708000 & 0.000202000 & 6 & 6.972045000 & 1.045531000 & 0.000060000 \\
\hline 6 & 3.610001000 & 2.260948000 & 0.000028000 & 6 & 5.602849000 & 1.152725000 & 0.000026000 \\
\hline 6 & -3.154507000 & -0.184771000 & 0.000025000 & 6 & -3.997616000 & 1.622389000 & 0.000004000 \\
\hline 6 & -4.057882000 & -1.255654000 & 0.000090000 & 6 & -3.394782000 & 0.357594000 & -0.000069000 \\
\hline 6 & -3.571604000 & 1.148521000 & -0.000053000 & 6 & -5.373502000 & 1.805442000 & 0.000180000 \\
\hline
\end{tabular}




\begin{tabular}{|c|c|c|c|c|c|c|c|}
\hline 6 & -5.010318000 & 1.341509000 & -0.000064000 & 6 & -6.148109000 & 0.646087000 & 0.000256000 \\
\hline 6 & -5.890992000 & 0.233888000 & -0.000002000 & 6 & -5.576676000 & -0.648420000 & 0.000165000 \\
\hline 6 & -5.435347000 & -1.083877000 & 0.000075000 & 6 & -4.136162000 & -0.826532000 & -0.000008000 \\
\hline 6 & -2.723446000 & 2.292837000 & -0.000117000 & 6 & -6.395478000 & -1.813650000 & 0.000236000 \\
\hline 6 & -3.251383000 & 3.557252000 & -0.000189000 & 6 & -5.846685000 & -3.069930000 & 0.000136000 \\
\hline 6 & -4.658292000 & 3.752416000 & -0.000201000 & 6 & -4.436135000 & -3.237833000 & -0.000046000 \\
\hline 6 & -5.507335000 & 2.675993000 & -0.000140000 & 6 & -3.605236000 & -2.148458000 & -0.000114000 \\
\hline 1 & 1.547827000 & -4.084209000 & -0.000077000 & 1 & 2.136526000 & 2.945113000 & 0.000025000 \\
\hline 1 & -3.213594000 & -4.382790000 & 0.000117000 & 1 & -0.570765000 & -1.363821000 & -0.000156000 \\
\hline 1 & -0.756099000 & -4.795355000 & 0.000014000 & 1 & 0.090907000 & 4.212421000 & 0.000076000 \\
\hline 1 & 5.189674000 & -2.918778000 & -0.000002000 & 1 & -2.390489000 & 4.434401000 & 0.000132000 \\
\hline 1 & 6.831227000 & -1.060028000 & 0.000263000 & 1 & 2.663535000 & -3.395125000 & -0.000120000 \\
\hline 1 & 7.387717000 & 1.310089000 & 0.000422000 & 1 & 5.143007000 & -3.451800000 & -0.000037000 \\
\hline 1 & 6.698194000 & 3.673531000 & 0.000473000 & 1 & 7.313781000 & -2.347140000 & 0.000043000 \\
\hline 1 & 4.289948000 & 4.283586000 & 0.000229000 & 1 & 8.674003000 & -0.295362000 & 0.000095000 \\
\hline 1 & 2.558662000 & 2.525400000 & -0.000088000 & 1 & 7.587072000 & 1.938361000 & 0.000082000 \\
\hline 1 & -6.957790000 & 0.431647000 & -0.000017000 & 1 & 5.125695000 & 2.126170000 & 0.000022000 \\
\hline 1 & -6.131230000 & -1.914367000 & 0.000117000 & 1 & -5.836063000 & 2.785216000 & 0.000255000 \\
\hline 1 & -2.593786000 & 4.419364000 & -0.000237000 & 1 & -7.230192000 & 0.725028000 & 0.000391000 \\
\hline 1 & -5.057456000 & 4.760299000 & -0.000258000 & 1 & -7.473456000 & -1.688295000 & 0.000367000 \\
\hline 1 & -6.581931000 & 2.827589000 & -0.000149000 & 1 & 8190000 & 3825000 & 0.000190000 \\
\hline 1 & -1.649663000 & 2.151508000 & -0.000109000 & 1 & -4.018651000 & -4.238481000 & -0.000134000 \\
\hline 1 & 0.008641000 & 0.766136000 & -0.000032000 & 1 & -2.530622000 & -2.283537000 & -0.000264000 \\
\hline \multicolumn{4}{|c|}{$E=-1153.00652405$} & \multicolumn{4}{|c|}{$E=-1153.00649268$} \\
\hline \multicolumn{4}{|c|}{$\mathrm{NC} 22$} & \multicolumn{4}{|c|}{$\mathrm{NC} 23$} \\
\hline 6 & -0.400447000 & 4.210938000 & 0.000000000 & 6 & -5.455784000 & 0.552553000 & 0.000005000 \\
\hline 6 & -1.260244000 & 5.378530000 & 0.000000000 & 6 & -5.601524000 & -0.875205000 & -0.000047000 \\
\hline 6 & -2.688064000 & 5.231767000 & 0.000000000 & 6 & -6.616588000 & 1.349620000 & 0.000185000 \\
\hline 6 & -3.292704000 & 3.913839000 & 0.000000000 & 6 & -4.138075000 & 1.157920000 & -0.000104000 \\
\hline 6 & -2.451290000 & 2.857194000 & 0.000000000 & 6 & -3.080461000 & 0.317209000 & -0.000353000 \\
\hline 6 & -1.009299000 & 3.004864000 & 0.000000000 & 6 & -3.228340000 & -1.124809000 & -0.000357000 \\
\hline 6 & -2.274115000 & 1.369831000 & 0.000000000 & 6 & -1.592705000 & 0.136556000 & -0.000273000 \\
\hline 6 & -0.884064000 & 1.511563000 & 0.000000000 & 6 & -1.735830000 & -1.252512000 & -0.000377000 \\
\hline 6 & -2.913165000 & 0.121349000 & 0.000000000 & 6 & -0.345750000 & 0.783001000 & -0.000096000 \\
\hline 6 & -2.091794000 & -0.990561000 & 0.000000000 & 6 & 0.803237000 & -0.130160000 & -0.000040000 \\
\hline 6 & -0.666856000 & -0.885608000 & 0.000000000 & 6 & 2.041651000 & 0.511496000 & 0.000282000 \\
\hline 6 & 0.000000000 & 0.420389000 & 0.000000000 & 6 & 2.173128000 & 1.905965000 & 0.000333000 \\
\hline 6 & -0.715022000 & 6.676773000 & 0.000000000 & 6 & 3.559075000 & 0.373024000 & 0.000315000 \\
\hline 6 & -1.524939000 & 7.800181000 & 0.000000000 & 6 & 3.681011000 & 1.767993000 & 0.000165000 \\
\hline 6 & -2.919511000 & 7.656978000 & 0.000000000 & 6 & 4.663244000 & -0.478181000 & 0.000280000 \\
\hline 6 & -3.484993000 & 6.392711000 & 0.000000000 & 6 & 5.952832000 & 0.192078000 & -0.000085000 \\
\hline 6 & 0.205058000 & -1.969746000 & 0.000000000 & 6 & -4.433535000 & -1.734524000 & -0.000185000 \\
\hline 6 & 1.598855000 & -1.834378000 & 0.000000000 & 6 & -6.899886000 & -1.420391000 & 0.000064000 \\
\hline 6 & 2.236329000 & -0.599982000 & 0.000000000 & 6 & 0.613254000 & -1.545959000 & -0.000109000 \\
\hline 6 & 1.410304000 & 0.525234000 & 0.000000000 & 6 & -0.640803000 & -2.127849000 & -0.000295000 \\
\hline 6 & 0.349276000 & -3.481307000 & 0.000000000 & 6 & -0.174312000 & 2.186092000 & 0.000002000 \\
\hline 6 & 1.742501000 & -3.344154000 & 0.000000000 & 6 & 1.090892000 & 2.774310000 & 0.000229000 \\
\hline 6 & -0.281023000 & -4.716372000 & 0.000000000 & 6 & 4.903446000 & 2.420061000 & -0.000319000 \\
\hline 6 & 0.556089000 & -5.836125000 & 0.000000000 & 6 & 4.626499000 & -1.903322000 & 0.000425000 \\
\hline
\end{tabular}




\begin{tabular}{|c|c|c|c|c|c|c|c|}
\hline 6 & 1.965368000 & -5.731049000 & 0.000000000 & 6 & 6.036200000 & 1.602307000 & -0.000401000 \\
\hline 6 & 2.611469000 & -4.430727000 & 0.000000000 & 6 & 7.126784000 & -0.615959000 & -0.000164000 \\
\hline 6 & 2.798780000 & -6.886908000 & 0.000000000 & 6 & -8.023296000 & -0.610493000 & 0.000237000 \\
\hline 6 & 4.164984000 & -6.777368000 & 0.000000000 & 6 & -7.880828000 & 0.784157000 & 0.000299000 \\
\hline 6 & 4.789387000 & -5.499313000 & 0.000000000 & 6 & 7.049513000 & -1.983783000 & 0.000042000 \\
\hline 6 & 4.035046000 & -4.356577000 & 0.000000000 & 6 & 5.784783000 & -2.633353000 & 0.000325000 \\
\hline 1 & 0.675690000 & 4.344973000 & 0.000000000 & 1 & -9.012087000 & -1.055049000 & 0.000330000 \\
\hline 1 & -4.373514000 & 3.824389000 & 0.000000000 & 1 & -8.759085000 & 1.419759000 & 0.000433000 \\
\hline 1 & -3.991735000 & 0.018822000 & 0.000000000 & 1 & -6.506761000 & 2.429408000 & 0.000228000 \\
\hline 1 & -2.526737000 & -1.983671000 & 0.000000000 & 1 & -7.011016000 & -2.500017000 & 0.000009000 \\
\hline 1 & 0.364626000 & 6.788059000 & 0.000000000 & 1 & -4.051407000 & 2.238898000 & -0.000008000 \\
\hline 1 & -1.080591000 & 8.789038000 & 0.000000000 & 1 & -4.564975000 & -2.811031000 & -0.000167000 \\
\hline 1 & -3.555374000 & 8.535085000 & 0.000000000 & 1 & 1.494068000 & -2.176272000 & -0.000017000 \\
\hline 1 & -4.564772000 & 6.282951000 & 0.000000000 & 1 & -0.754872000 & -3.205243000 & -0.000325000 \\
\hline 1 & 3.314624000 & -0.495009000 & 0.000000000 & 1 & -1.059901000 & 2.811037000 & -0.000122000 \\
\hline 1 & 1.850663000 & 1.515644000 & 0.000000000 & 1 & 1.203780000 & 3.851875000 & 0.000207000 \\
\hline 1 & -1.358409000 & -4.830305000 & 0.000000000 & 1 & 4.995252000 & 3.499601000 & -0.000453000 \\
\hline 1 & 0.118636000 & -6.828861000 & 0.000000000 & 1 & 7.022238000 & 2.054707000 & -0.000689000 \\
\hline 1 & 2.329292000 & -7.865236000 & 0.000000000 & 1 & 8.093646000 & -0.123301000 & -0.000408000 \\
\hline 1 & 4.780587000 & -7.669736000 & 0.000000000 & 1 & 7.954991000 & -2.579770000 & -0.000025000 \\
\hline 1 & 5.871750000 & -5.436124000 & 0.000000000 & 1 & 5.742675000 & 744000 & 57000 \\
\hline 1 & 4.509753000 & -3.381816000 & 0.000000000 & 1 & 3.666459000 & -2.404921000 & 0.000643000 \\
\hline \multicolumn{4}{|c|}{$E=-1153.00410495$} & \multicolumn{4}{|c|}{$E=-1153.00312558$} \\
\hline \multicolumn{4}{|c|}{$\mathrm{NC} 24$} & \multicolumn{4}{|c|}{$\mathrm{NC} 25$} \\
\hline 6 & 3.150789000 & 2.966171000 & 0.000000000 & 6 & 4.358960000 & -0.020886000 & 0.004343000 \\
\hline 6 & 4.143363000 & 1.906651000 & 0.000000000 & 6 & 3.379260000 & -1.021914000 & -0.302553000 \\
\hline 6 & 1.829620000 & 2.532477000 & 0.000000000 & 6 & 3.945971000 & 1.338977000 & 0.287823000 \\
\hline 6 & 1.467278000 & 1.180306000 & 0.000000000 & 6 & 2.619063000 & 1.586369000 & 0.284628000 \\
\hline 6 & 0.365905000 & 2.927502000 & 0.000000000 & 6 & 1.621389000 & 0.568907000 & 0.020039000 \\
\hline 6 & 0.000000000 & 1.576569000 & 0.000000000 & 6 & 1.527788000 & 2.601294000 & 0.369579000 \\
\hline 6 & -1.329831000 & 1.160214000 & 0.000000000 & 6 & 0.552387000 & 1.622527000 & 0.159048000 \\
\hline 6 & -2.324985000 & 2.239694000 & 0.000000000 & 6 & -0.820601000 & 1.940353000 & 0.052021000 \\
\hline 6 & -1.862549000 & -0.143776000 & 0.000000000 & 6 & -1.117528000 & 3.382182000 & 0.072482000 \\
\hline 6 & -3.236558000 & -0.390031000 & 0.000000000 & 6 & -1.939404000 & 1.112456000 & -0.092271000 \\
\hline 6 & -1.572522000 & -1.614952000 & 0.000000000 & 6 & -3.218497000 & 1.630728000 & -0.337931000 \\
\hline 6 & -2.999629000 & -1.868962000 & 0.000000000 & 6 & -2.530110000 & -0.296804000 & -0.088829000 \\
\hline 6 & 3.579954000 & 4.326165000 & 0.000000000 & 6 & -3.789229000 & 0.233358000 & -0.394528000 \\
\hline 6 & 2.404907000 & 0.159102000 & 0.000000000 & 6 & -2.341654000 & -1.654621000 & 0.170227000 \\
\hline 6 & -0.559061000 & 3.962543000 & 0.000000000 & 6 & -3.515926000 & -2.488359000 & -0.026932000 \\
\hline 6 & -4.198068000 & 0.628846000 & 0.000000000 & 6 & 1.965305000 & -0.698209000 & -0.305338000 \\
\hline 6 & -3.523021000 & -3.113209000 & 0.000000000 & 6 & 1.248553000 & 3.970503000 & 0.454888000 \\
\hline 6 & -2.584065000 & -4.218387000 & 0.000000000 & 6 & -3.508756000 & 2.985039000 & -0.366579000 \\
\hline 6 & -1.170813000 & -3.969854000 & 0.000000000 & 6 & -4.922218000 & -0.544179000 & -0.566846000 \\
\hline 6 & -0.661169000 & -2.612291000 & 0.000000000 & 6 & -1.141393000 & -2.255786000 & 0.646336000 \\
\hline 6 & -3.035454000 & -5.551784000 & 0.000000000 & 6 & 3.818854000 & -2.325800000 & -0.602279000 \\
\hline 6 & -0.292873000 & -5.070595000 & 0.000000000 & 6 & 5.719528000 & -0.382056000 & 0.001561000 \\
\hline 6 & 5.517934000 & 2.281844000 & 0.000000000 & 6 & -0.073996000 & 4.331080000 & 0.289413000 \\
\hline 6 & 3.746978000 & 0.550679000 & 0.000000000 & 6 & -2.436331000 & 3.845515000 & -0.134643000 \\
\hline 6 & -1.904293000 & 3.589910000 & 0.000000000 & 6 & -4.754308000 & -1.920815000 & -0.399503000 \\
\hline
\end{tabular}




\begin{tabular}{|c|c|c|c|c|c|c|c|}
\hline 6 & -3.717203000 & 1.925785000 & 0.000000000 & 6 & -3.385355000 & -3.889782000 & 0.197426000 \\
\hline 6 & 5.893729000 & 3.599969000 & 0.000000000 & 6 & -2.203480000 & -4.435073000 & 0.625554000 \\
\hline 6 & 4.914402000 & 4.632054000 & 0.000000000 & 6 & -1.075076000 & -3.605452000 & 0.869348000 \\
\hline 6 & -2.147177000 & -6.614641000 & 0.000000000 & 6 & 5.164905000 & -2.652376000 & -0.598197000 \\
\hline 6 & -0.766537000 & -6.372171000 & 0.000000000 & 6 & 6.121759000 & -1.674524000 & -0.292398000 \\
\hline 1 & 6.944929000 & 3.864821000 & 0.000000000 & 1 & 4.698499000 & 2.097884000 & 0.472306000 \\
\hline 1 & 5.234537000 & 5.668190000 & 0.000000000 & 1 & 1.256353000 & -1.468927000 & -0.579494000 \\
\hline 1 & 2.833435000 & 5.112435000 & 0.000000000 & 1 & 2.019875000 & 4.715033000 & 0.609632000 \\
\hline 1 & 6.269498000 & 1.499046000 & 0.000000000 & 1 & -0.350362000 & 5.380110000 & 0.310084000 \\
\hline 1 & 2.136911000 & -0.889933000 & 0.000000000 & 1 & -2.605993000 & 4.916770000 & -0.114474000 \\
\hline 1 & 4.522725000 & -0.207806000 & 0.000000000 & 1 & -4.507414000 & 3.368619000 & -0.536860000 \\
\hline 1 & -0.273445000 & 5.007473000 & 0.000000000 & 1 & -5.891080000 & -0.125979000 & -0.812442000 \\
\hline 1 & -2.668831000 & 4.359554000 & 0.000000000 & 1 & -5.601224000 & -2.584934000 & -0.535511000 \\
\hline 1 & -4.417285000 & 2.754815000 & 0.000000000 & 1 & -4.252331000 & -4.522162000 & 0.035934000 \\
\hline 1 & -5.262378000 & 0.427367000 & 0.000000000 & 1 & -2.128326000 & -5.502737000 & 0.797862000 \\
\hline 1 & -4.587831000 & -3.319030000 & 0.000000000 & 1 & -0.159233000 & -4.047934000 & 1.245192000 \\
\hline 1 & 0.410696000 & -2.452689000 & 0.000000000 & 1 & -0.290926000 & -1.622629000 & 0.860371000 \\
\hline 1 & -4.104435000 & -5.739552000 & 0.000000000 & 1 & 3.078824000 & -3.083070000 & -0.840691000 \\
\hline 1 & -2.519777000 & -7.632710000 & 0.000000000 & 1 & 5.477931000 & -3.663600000 & -0.832333000 \\
\hline 1 & -0.069477000 & -7.202493000 & 0.000000000 & 1 & 7.175701000 & -1.928549000 & -0.288734000 \\
\hline 1 & 0.776421000 & -4.884447000 & 0.000000000 & 1 & 6.460540000 & 0.376186000 & 0.233818000 \\
\hline \multicolumn{4}{|c|}{$E=-1153.00251904$} & \multicolumn{4}{|c|}{$E=-1152.99781416$} \\
\hline \multicolumn{4}{|c|}{ NC26 } & \multicolumn{4}{|c|}{$\mathrm{NC} 27$} \\
\hline 6 & 5.116716000 & 0.377831000 & -0.000059000 & 6 & -5.820483000 & 0.203955000 & 0.000071000 \\
\hline 6 & 5.903023000 & -0.843607000 & 0.000125000 & 6 & -4.452625000 & 0.697251000 & 0.000238000 \\
\hline 6 & 3.739005000 & 0.188454000 & 0.000014000 & 6 & -3.471527000 & -0.291880000 & 0.000334000 \\
\hline 6 & 3.143353000 & -1.078920000 & 0.000100000 & 6 & -3.776521000 & -1.658979000 & 0.000228000 \\
\hline 6 & 2.367050000 & 0.836830000 & -0.000088000 & 6 & -1.985013000 & -0.631950000 & 0.000057000 \\
\hline 6 & 1.771135000 & -0.425846000 & -0.000049000 & 6 & -2.298483000 & -1.992769000 & 0.000238000 \\
\hline 6 & 0.390716000 & -0.623120000 & -0.000105000 & 6 & -0.670685000 & -0.155261000 & -0.000225000 \\
\hline 6 & -0.390722000 & 0.623153000 & -0.000135000 & 6 & 0.349923000 & -1.216204000 & -0.000268000 \\
\hline 6 & -1.771140000 & 0.425871000 & -0.000181000 & 6 & 1.662609000 & -0.744981000 & -0.000694000 \\
\hline 6 & -2.367043000 & -0.836806000 & -0.000217000 & 6 & 1.993636000 & 0.610669000 & -0.000791000 \\
\hline 6 & -3.143358000 & 1.078941000 & 0.000103000 & 6 & 3.137985000 & -1.110509000 & -0.000274000 \\
\hline 6 & -3.739002000 & -0.188446000 & 0.000018000 & 6 & 3.467794000 & 0.250411000 & -0.000218000 \\
\hline 6 & -5.116705000 & -0.377838000 & -0.000053000 & 6 & 4.779589000 & 0.711801000 & -0.000004000 \\
\hline 6 & -5.903035000 & 0.843590000 & 0.000144000 & 6 & 5.794755000 & -0.327320000 & 0.000204000 \\
\hline 6 & 5.782812000 & 1.638763000 & -0.000219000 & 6 & -4.227049000 & 2.105584000 & 0.000248000 \\
\hline 6 & 3.880517000 & -2.252128000 & 0.000315000 & 6 & -5.073961000 & -2.142922000 & 0.000003000 \\
\hline 6 & 1.632281000 & 2.021815000 & -0.000003000 & 6 & -1.338086000 & -3.001253000 & 0.000268000 \\
\hline 6 & 0.247714000 & 1.892823000 & -0.000064000 & 6 & -0.010126000 & -2.590252000 & 0.000003000 \\
\hline 6 & -1.632289000 & -2.021790000 & -0.000034000 & 6 & 1.034227000 & 1.621529000 & -0.000417000 \\
\hline 6 & -0.247706000 & -1.892790000 & -0.000035000 & 6 & -0.295765000 & 1.215058000 & -0.000262000 \\
\hline 6 & -3.880562000 & 2.252145000 & 0.000294000 & 6 & 4.095319000 & -2.112254000 & -0.000151000 \\
\hline 6 & -5.782775000 & -1.638779000 & -0.000229000 & 6 & 5.179194000 & 2.080662000 & -0.000023000 \\
\hline 6 & 7.322940000 & -0.719304000 & 0.000107000 & 6 & -6.876772000 & 1.161447000 & -0.000024000 \\
\hline 6 & 5.272194000 & -2.107172000 & 0.000302000 & 6 & -6.089504000 & -1.181804000 & -0.000071000 \\
\hline 6 & -5.272227000 & 2.107167000 & 0.000304000 & 6 & 5.429869000 & -1.691605000 & 0.000119000 \\
\hline 6 & -7.322942000 & 0.719268000 & 0.000145000 & 6 & 7.160964000 & 0.079159000 & 0.000432000 \\
\hline
\end{tabular}




\begin{tabular}{|c|c|c|c|c|c|c|c|}
\hline 6 & 7.928468000 & 0.509986000 & -0.000066000 & 6 & -6.618246000 & 2.506555000 & 0.000006000 \\
\hline 6 & 7.150302000 & 1.701339000 & -0.000236000 & 6 & -5.277836000 & 2.982606000 & 0.000139000 \\
\hline 6 & -7.928450000 & -0.510038000 & -0.000039000 & 6 & 7.507600000 & 1.404959000 & 0.000420000 \\
\hline 6 & -7.150269000 & -1.701375000 & -0.000236000 & 6 & 6.506391000 & 2.416171000 & 0.000182000 \\
\hline 1 & 7.922561000 & -1.623818000 & 0.000228000 & 1 & -7.436372000 & 3.217764000 & -0.000099000 \\
\hline 1 & 9.010212000 & 0.581850000 & -0.000087000 & 1 & -7.900596000 & 0.801512000 & -0.000132000 \\
\hline 1 & 7.650629000 & 2.663223000 & -0.000383000 & 1 & -3.208693000 & 2.474689000 & 0.000353000 \\
\hline 1 & 5.188735000 & 2.545792000 & -0.000353000 & 1 & -5.092582000 & 4.050899000 & 0.000156000 \\
\hline 1 & 3.426203000 & -3.235678000 & 0.000393000 & 1 & -7.126589000 & -1.500417000 & -0.000188000 \\
\hline 1 & 5.900426000 & -2.991647000 & 0.000434000 & 1 & -5.308393000 & -3.200739000 & -0.000022000 \\
\hline 1 & 2.097867000 & 3.000094000 & 0.000007000 & 1 & -1.595844000 & -4.053661000 & 0.000402000 \\
\hline 1 & -0.376515000 & 2.779041000 & -0.000064000 & 1 & 0.784587000 & -3.327387000 & -0.000002000 \\
\hline 1 & -2.097866000 & -3.000070000 & 0.000008000 & 1 & 1.290798000 & 2.674153000 & -0.000287000 \\
\hline 1 & 0.376520000 & -2.779009000 & 0.000034000 & 1 & -1.080938000 & 1.960779000 & -0.000039000 \\
\hline 1 & -3.426266000 & 3.235706000 & 0.000348000 & 1 & 3.847417000 & -3.166937000 & -0.000174000 \\
\hline 1 & -5.900481000 & 2.991629000 & 0.000433000 & 1 & 6.222405000 & -2.432395000 & 0.000280000 \\
\hline 1 & -7.922589000 & 1.623765000 & 0.000287000 & 1 & 7.929848000 & -0.686655000 & 0.000604000 \\
\hline 1 & -9.010193000 & -0.581907000 & -0.000048000 & 1 & 8.552968000 & 1.692283000 & 0.000580000 \\
\hline 1 & -7.650573000 & -2.663270000 & -0.000393000 & 1 & 6.803716000 & 3.458842000 & 0.000160000 \\
\hline 1 & -5.188690000 & -2.545804000 & -0.000380000 & 1 & 4.415213000 & 2.850039000 & -0.000194000 \\
\hline \multicolumn{4}{|c|}{$E=-1152.99025804$} & \multicolumn{4}{|c|}{$E=-1152.98945078$} \\
\hline \multicolumn{4}{|c|}{ NC28 } & \multicolumn{4}{|c|}{ NC29 } \\
\hline 6 & 4.502665000 & 0.300872000 & 0.000011000 & 6 & 0.000000000 & 4.524373000 & -0.312592000 \\
\hline 6 & 5.710016000 & -0.509658000 & 0.000469000 & 6 & 0.000000000 & 4.538110000 & -1.765344000 \\
\hline 6 & 3.310734000 & -0.420866000 & -0.000257000 & 6 & 0.000000000 & 3.258990000 & 0.261693000 \\
\hline 6 & 3.276067000 & -1.821089000 & -0.000142000 & 6 & 0.000000000 & 2.076846000 & -0.488207000 \\
\hline 6 & 1.785382000 & -0.392030000 & -0.000788000 & 6 & 0.000000000 & 2.445506000 & 1.543842000 \\
\hline 6 & 1.761384000 & -1.787469000 & -0.000816000 & 6 & 0.000000000 & 1.266565000 & 0.799325000 \\
\hline 6 & 0.625525000 & 0.389294000 & -0.000605000 & 6 & 0.000000000 & 0.000000000 & 1.393707000 \\
\hline 6 & -0.625525000 & -0.389300000 & -0.000678000 & 6 & 0.000000000 & 0.000000000 & 2.866164000 \\
\hline 6 & -1.785378000 & 0.392029000 & -0.000656000 & 6 & 0.000000000 & -1.266565000 & 0.799325000 \\
\hline 6 & -1.761370000 & 1.787462000 & -0.000485000 & 6 & 0.000000000 & -2.445506000 & 1.543842000 \\
\hline 6 & -3.310734000 & 0.420868000 & -0.000136000 & 6 & 0.000000000 & -2.076846000 & -0.488207000 \\
\hline 6 & -3.276070000 & 1.821083000 & -0.000089000 & 6 & 0.000000000 & -3.258990000 & 0.261693000 \\
\hline 6 & -4.502668000 & -0.300874000 & 0.000158000 & 6 & 0.000000000 & -4.524373000 & -0.312592000 \\
\hline 6 & -5.710008000 & 0.509651000 & 0.000400000 & 6 & 0.000000000 & -4.538110000 & -1.765344000 \\
\hline 6 & 4.626310000 & 1.721682000 & -0.000104000 & 6 & 0.000000000 & 5.761261000 & 0.398127000 \\
\hline 6 & 4.417218000 & -2.605412000 & 0.000256000 & 6 & 0.000000000 & 2.075918000 & -1.873687000 \\
\hline 6 & -0.603241000 & -1.809086000 & -0.000592000 & 6 & 0.000000000 & 2.467274000 & 2.936493000 \\
\hline 6 & 0.584730000 & -2.531014000 & -0.000582000 & 6 & 0.000000000 & -2.467274000 & 2.936493000 \\
\hline 6 & 0.603249000 & 1.809082000 & -0.000355000 & 6 & 0.000000000 & -2.075918000 & -1.873687000 \\
\hline 6 & -0.584718000 & 2.531015000 & -0.000196000 & 6 & 0.000000000 & -5.761261000 & 0.398127000 \\
\hline 6 & -4.417219000 & 2.605419000 & 0.000036000 & 6 & 0.000000000 & 5.805048000 & -2.418561000 \\
\hline 6 & -4.626319000 & -1.721679000 & 0.000109000 & 6 & 0.000000000 & 3.329728000 & -2.494858000 \\
\hline 6 & 6.967250000 & 0.162553000 & 0.000793000 & 6 & 0.000000000 & 1.230224000 & 3.573520000 \\
\hline 6 & 5.635450000 & -1.919290000 & 0.000581000 & 6 & 0.000000000 & -1.230224000 & 3.573520000 \\
\hline 6 & -5.635451000 & 1.919293000 & 0.000300000 & 6 & 0.000000000 & -3.329728000 & -2.494858000 \\
\hline 6 & -6.967249000 & -0.162544000 & 0.000692000 & 6 & 0.000000000 & -5.805048000 & -2.418561000 \\
\hline 6 & 7.043453000 & 1.530096000 & 0.000677000 & 6 & 0.000000000 & 6.973141000 & -1.702325000 \\
\hline
\end{tabular}




\begin{tabular}{|c|c|c|c|c|c|c|c|}
\hline 6 & 5.858726000 & 2.317273000 & 0.000211000 & 6 & 0.000000000 & 6.950596000 & -0.279251000 \\
\hline 6 & -7.043461000 & -1.530094000 & 0.000698000 & 6 & 0.000000000 & -6.950596000 & -0.279251000 \\
\hline 6 & -5.858743000 & -2.317270000 & 0.000382000 & 6 & 0.000000000 & -6.973141000 & -1.702325000 \\
\hline 1 & 8.009847000 & 2.021232000 & 0.000941000 & 1 & 0.000000000 & 7.926338000 & -2.218715000 \\
\hline 1 & 5.938187000 & 3.398605000 & 0.000105000 & 1 & 0.000000000 & 7.887146000 & 0.267056000 \\
\hline 1 & 3.728482000 & 2.327527000 & -0.000463000 & 1 & 0.000000000 & 5.743329000 & 1.482202000 \\
\hline 1 & 7.872870000 & -0.435447000 & 0.001152000 & 1 & 0.000000000 & 5.829064000 & -3.503513000 \\
\hline 1 & 4.387825000 & -3.688484000 & 0.000291000 & 1 & 0.000000000 & 1.168761000 & -2.464718000 \\
\hline 1 & 6.564510000 & -2.479513000 & 0.000892000 & 1 & 0.000000000 & 3.387025000 & -3.578239000 \\
\hline 1 & -1.549001000 & -2.336281000 & -0.000494000 & 1 & 0.000000000 & 3.386486000 & 3.509602000 \\
\hline 1 & 0.577402000 & -3.614487000 & -0.000408000 & 1 & 0.000000000 & 1.189472000 & 4.657660000 \\
\hline 1 & 1.549009000 & 2.336275000 & -0.000296000 & 1 & 0.000000000 & -1.189472000 & 4.657660000 \\
\hline 1 & -0.577399000 & 3.614488000 & 0.000025000 & 1 & 0.000000000 & -3.386486000 & 3.509602000 \\
\hline 1 & -4.387819000 & 3.688489000 & 0.000052000 & 1 & 0.000000000 & -3.387025000 & -3.578239000 \\
\hline 1 & -6.564511000 & 2.479516000 & 0.000453000 & 1 & 0.000000000 & -1.168761000 & -2.464718000 \\
\hline 1 & -7.872869000 & 0.435452000 & 0.000902000 & 1 & 0.000000000 & -5.743329000 & 1.482202000 \\
\hline 1 & -8.009864000 & -2.021213000 & 0.000924000 & 1 & 0.000000000 & -7.887146000 & 0.267056000 \\
\hline 1 & -5.938195000 & -3.398603000 & 0.000344000 & 1 & 0.000000000 & -7.926338000 & -2.218715000 \\
\hline 1 & -3.728500000 & -2.327538000 & -0.000159000 & 1 & 0.000000000 & -5.829064000 & -3.503513000 \\
\hline \multicolumn{4}{|c|}{$E=-1152.98837920$} & \multicolumn{4}{|c|}{$E=-1152.98829580$} \\
\hline \multicolumn{4}{|c|}{ NC30 } & \multicolumn{4}{|c|}{ NC31 } \\
\hline 6 & 3.272585000 & 2.408980000 & 0.000000000 & 6 & -0.395324000 & 3.264577000 & 0.974768000 \\
\hline 6 & 4.193249000 & 1.289960000 & 0.000000000 & 6 & 0.000000000 & 2.151244000 & 0.221228000 \\
\hline 6 & 1.926427000 & 2.064562000 & 0.000000000 & 6 & 0.4809 & 2.274324000 & -1.082765000 \\
\hline 6 & 1.449955000 & 0.749220000 & 0.000000000 & 6 & -0.147868000 & 1.267955000 & 1.460934000 \\
\hline 6 & 0.520808000 & 2.589649000 & 0.000000000 & 6 & 0.384661000 & 3.611133000 & -1.649883000 \\
\hline 6 & 0.000000000 & 1.290502000 & 0.000000000 & 6 & -0.459690000 & 2.400429000 & 2.215184000 \\
\hline 6 & -1.390298000 & 1.047088000 & 0.000000000 & 6 & 0.000000000 & 0.000000000 & 2.045919000 \\
\hline 6 & -2.214466000 & 2.282340000 & 0.000000000 & 6 & 0.000000000 & 0.000000000 & 3.521602000 \\
\hline 6 & -2.148528000 & -0.145664000 & 0.000000000 & 6 & 0.147868000 & -1.267955000 & 1.460934000 \\
\hline 6 & -3.547651000 & -0.123261000 & 0.000000000 & 6 & 0.459690000 & -2.400429000 & 2.215184000 \\
\hline 6 & -2.212647000 & -1.691879000 & 0.000000000 & 6 & 0.000000000 & -2.151244000 & 0.221228000 \\
\hline 6 & -3.609011000 & -1.623471000 & 0.000000000 & 6 & 0.395324000 & -3.264577000 & 0.974768000 \\
\hline 6 & -1.547761000 & -2.919305000 & 0.000000000 & 6 & -0.480902000 & -2.274324000 & -1.082765000 \\
\hline 6 & -2.401993000 & -4.096958000 & 0.000000000 & 6 & -0.384661000 & -3.611133000 & -1.649883000 \\
\hline 6 & 3.788606000 & 3.738908000 & 0.000000000 & 6 & -0.470204000 & 4.545919000 & 0.458091000 \\
\hline 6 & 2.329882000 & -0.325451000 & 0.000000000 & 6 & -0.545286000 & 2.403100000 & 3.603732000 \\
\hline 6 & -0.241532000 & 3.750626000 & 0.000000000 & 6 & 0.545286000 & -2.403100000 & 3.603732000 \\
\hline 6 & -4.327080000 & 1.024760000 & 0.000000000 & 6 & 0.470204000 & -4.545919000 & 0.458091000 \\
\hline 6 & -4.439360000 & -2.730763000 & 0.000000000 & 6 & -1.094870000 & -1.242379000 & -1.851820000 \\
\hline 6 & -0.138499000 & -3.101929000 & 0.000000000 & 6 & 1.094870000 & 1.242379000 & -1.851820000 \\
\hline 6 & 5.590328000 & 1.572874000 & 0.000000000 & 6 & -0.101781000 & 4.691055000 & -0.882894000 \\
\hline 6 & 3.697699000 & -0.029067000 & 0.000000000 & 6 & 0.840307000 & 3.804500000 & -2.986695000 \\
\hline 6 & -1.613945000 & 3.566245000 & 0.000000000 & 6 & -0.282746000 & 1.192945000 & 4.235264000 \\
\hline 6 & -3.630093000 & 2.222011000 & 0.000000000 & 6 & 0.282746000 & -1.192945000 & 4.235264000 \\
\hline 6 & -3.807021000 & -3.975343000 & 0.000000000 & 6 & 0.101781000 & -4.691055000 & -0.882894000 \\
\hline 6 & -1.776074000 & -5.377684000 & 0.000000000 & 6 & -0.840307000 & -3.804500000 & -2.986695000 \\
\hline 6 & 6.050400000 & 2.863367000 & 0.000000000 & 6 & -1.399779000 & -2.779200000 & -3.702512000 \\
\hline 6 & 5.139853000 & 3.957463000 & 0.000000000 & 6 & -1.543274000 & -1.489326000 & -3.121334000 \\
\hline
\end{tabular}




\begin{tabular}{|c|c|c|c|c|c|c|c|}
\hline 6 & -0.412332000 & -5.507168000 & 0.000000000 & 6 & 1.543274000 & 1.489326000 & -3.121334000 \\
\hline 6 & 0.416824000 & -4.352749000 & 0.000000000 & 6 & 1.399779000 & 2.779200000 & -3.702512000 \\
\hline 1 & 7.116611000 & 3.059550000 & 0.000000000 & 1 & -0.780778000 & 5.400626000 & 1.047215000 \\
\hline 1 & 5.526410000 & 4.970432000 & 0.000000000 & 1 & -0.154114000 & 5.668429000 & -1.350727000 \\
\hline 1 & 3.095626000 & 4.572677000 & 0.000000000 & 1 & -0.759556000 & 3.297822000 & 4.175621000 \\
\hline 1 & 6.289002000 & 0.742488000 & 0.000000000 & 1 & -0.282860000 & 1.144266000 & 5.319059000 \\
\hline 1 & 2.022520000 & -1.359056000 & 0.000000000 & 1 & 0.282860000 & -1.144266000 & 5.319059000 \\
\hline 1 & 4.412414000 & -0.845332000 & 0.000000000 & 1 & 0.759556000 & -3.297822000 & 4.175621000 \\
\hline 1 & 0.196092000 & 4.741151000 & 0.000000000 & 1 & 0.780778000 & -5.400626000 & 1.047215000 \\
\hline 1 & -2.272966000 & 4.427814000 & 0.000000000 & 1 & 0.154114000 & -5.668429000 & -1.350727000 \\
\hline 1 & -4.172160000 & 3.161560000 & 0.000000000 & 1 & -0.754146000 & -4.793959000 & -3.424033000 \\
\hline 1 & -5.409827000 & 0.997809000 & 0.000000000 & 1 & -1.751281000 & -2.950902000 & -4.713667000 \\
\hline 1 & -5.520049000 & -2.652291000 & 0.000000000 & 1 & -2.014437000 & -0.696876000 & -3.691657000 \\
\hline 1 & -4.401708000 & -4.882564000 & 0.000000000 & 1 & -1.217639000 & -0.261041000 & -1.412173000 \\
\hline 1 & -2.408360000 & -6.259693000 & 0.000000000 & 1 & 1.217639000 & 0.261041000 & -1.412173000 \\
\hline 1 & 0.041119000 & -6.491783000 & 0.000000000 & 1 & 2.014437000 & 0.696876000 & -3.691657000 \\
\hline 1 & 1.494898000 & -4.469908000 & 0.000000000 & 1 & 1.751281000 & 2.950902000 & -4.713667000 \\
\hline 1 & 0.480424000 & -2.220026000 & 0.000000000 & 1 & 0.754146000 & 4.793959000 & -3.424033000 \\
\hline \multicolumn{4}{|c|}{$E=-1152.98125191$} & \multicolumn{4}{|c|}{$E=-1152.97868604$} \\
\hline \multicolumn{4}{|c|}{ NP1 } & \multicolumn{4}{|c|}{ NP2 } \\
\hline 6 & 0.000000000 & 0.795127000 & 0.000000000 & 6 & 0.933729000 & -0.671246000 & 0.000000000 \\
\hline 6 & -1.150627000 & -0.090341000 & 0.000000000 & 6 & 0.000000000 & 0.381140000 & 0.000000000 \\
\hline 6 & -0.541598000 & 2.152990000 & 0.000000000 & 6 & 2.265603000 & -0.048705000 & 0.000000000 \\
\hline 6 & -2.008974000 & 2.042457000 & 0.000000000 & 6 & 2.066376000 & 1.400497000 & 0.000000000 \\
\hline 6 & -2.370074000 & 0.733473000 & 0.000000000 & 6 & 0.737224000 & 1.669235000 & 0.000000000 \\
\hline 6 & -0.219892000 & 3.472407000 & 0.000000000 & 6 & 3.598534000 & -0.292571000 & 0.000000000 \\
\hline 6 & -1.484074000 & 4.239309000 & 0.000000000 & 6 & 4.299695000 & 1.020696000 & 0.000000000 \\
\hline 6 & -2.552450000 & 3.393046000 & 0.000000000 & 6 & 3.390217000 & 2.028801000 & 0.000000000 \\
\hline 6 & -0.995526000 & -1.446735000 & 0.000000000 & 6 & -1.387766000 & 0.128055000 & 0.000000000 \\
\hline 6 & 0.320540000 & -2.013270000 & 0.000000000 & 6 & -1.814250000 & -1.249362000 & 0.000000000 \\
\hline 6 & 1.456934000 & -1.142660000 & 0.000000000 & 6 & -0.846829000 & -2.281306000 & 0.000000000 \\
\hline 6 & 1.262089000 & 0.278596000 & 0.000000000 & 6 & 0.510545000 & -2.004413000 & 0.000000000 \\
\hline 6 & 0.528819000 & -3.409797000 & 0.000000000 & 6 & -2.381307000 & 1.147909000 & 0.000000000 \\
\hline 6 & 1.804417000 & -3.939937000 & 0.000000000 & 6 & -3.716408000 & 0.831282000 & 0.000000000 \\
\hline 6 & 2.921644000 & -3.084936000 & 0.000000000 & 6 & -4.136875000 & -0.523053000 & 0.000000000 \\
\hline 6 & 2.748035000 & -1.714130000 & 0.000000000 & 6 & -3.207848000 & -1.532674000 & 0.000000000 \\
\hline 1 & -3.374272000 & 0.327242000 & 0.000000000 & 1 & 0.270200000 & 2.645676000 & 0.000000000 \\
\hline 1 & 0.764879000 & 3.920041000 & 0.000000000 & 1 & 4.102599000 & -1.250324000 & 0.000000000 \\
\hline 1 & -1.533938000 & 5.320331000 & 0.000000000 & 1 & 5.375587000 & 1.131652000 & 0.000000000 \\
\hline 1 & -3.596789000 & 3.668090000 & 0.000000000 & 1 & 3.598840000 & 3.088664000 & 0.000000000 \\
\hline 1 & -1.854435000 & -2.110792000 & 0.000000000 & 1 & -1.188022000 & -3.311175000 & 0.000000000 \\
\hline 1 & 2.134304000 & 0.924277000 & 0.000000000 & 1 & 1.232960000 & -2.812902000 & 0.000000000 \\
\hline 1 & -0.334591000 & -4.067389000 & 0.000000000 & 1 & -2.074399000 & 2.187418000 & 0.000000000 \\
\hline 1 & 1.946479000 & -5.014630000 & 0.000000000 & 1 & -4.459529000 & 1.620914000 & 0.000000000 \\
\hline 1 & 3.921484000 & -3.504250000 & 0.000000000 & 1 & -5.195648000 & -0.755596000 & 0.000000000 \\
\hline 1 & 3.611300000 & -1.056506000 & 0.000000000 & 1 & -3.526426000 & -2.570044000 & 0.000000000 \\
\hline \multicolumn{4}{|c|}{$E=-615.834249611$} & \multicolumn{4}{|c|}{$E=-615.825445075$} \\
\hline \multicolumn{4}{|c|}{ NP3 } & \multicolumn{4}{|c|}{ NP4 } \\
\hline
\end{tabular}




\begin{tabular}{|c|c|c|c|c|c|c|c|}
\hline 6 & -1.015120000 & 1.936459000 & 0.000000000 & 6 & -1.126289000 & -1.331266000 & 0.000000000 \\
\hline 6 & 0.658827000 & 0.327538000 & 0.000000000 & 6 & 1.002864000 & -0.407424000 & 0.000000000 \\
\hline 6 & -0.658827000 & -0.327538000 & 0.000000000 & 6 & 0.000000000 & 0.674171000 & 0.000000000 \\
\hline 6 & -1.642530000 & 0.609737000 & 0.000000000 & 6 & -1.247248000 & 0.132773000 & 0.000000000 \\
\hline 6 & 1.642530000 & -0.609737000 & 0.000000000 & 6 & 2.245577000 & 0.135009000 & 0.000000000 \\
\hline 6 & 1.015120000 & -1.936459000 & 0.000000000 & 6 & 2.097866000 & 1.601101000 & 0.000000000 \\
\hline 6 & -0.424776000 & -1.767555000 & 0.000000000 & 6 & 0.729770000 & 1.940111000 & 0.000000000 \\
\hline 6 & 0.424776000 & 1.767555000 & 0.000000000 & 6 & 0.281009000 & -1.676360000 & 0.000000000 \\
\hline 6 & 1.568707000 & -3.189794000 & 0.000000000 & 6 & 3.088222000 & 2.598740000 & 0.000000000 \\
\hline 6 & 0.724086000 & -4.340233000 & 0.000000000 & 6 & 2.706990000 & 3.924585000 & 0.000000000 \\
\hline 6 & -0.700131000 & -4.172084000 & 0.000000000 & 6 & 1.339728000 & 4.304427000 & 0.000000000 \\
\hline 6 & -1.253099000 & -2.855504000 & 0.000000000 & 6 & 0.316154000 & 3.293012000 & 0.000000000 \\
\hline 6 & 1.253243000 & -5.653449000 & 0.000000000 & 6 & -2.086873000 & -2.304870000 & 0.000000000 \\
\hline 6 & 0.424776000 & -6.755032000 & 0.000000000 & 6 & -1.702840000 & -3.682390000 & 0.000000000 \\
\hline 6 & -0.975853000 & -6.589242000 & 0.000000000 & 6 & -0.311509000 & -4.024599000 & 0.000000000 \\
\hline 6 & -1.522652000 & -5.323831000 & 0.000000000 & 6 & 0.671980000 & -2.984736000 & 0.000000000 \\
\hline 6 & -1.568707000 & 3.189794000 & 0.000000000 & 6 & -2.660780000 & -4.722200000 & 0.000000000 \\
\hline 6 & -0.724086000 & 4.340233000 & 0.000000000 & 6 & -2.274064000 & -6.046835000 & 0.000000000 \\
\hline 6 & 0.700131000 & 4.172084000 & 0.000000000 & 6 & -0.906327000 & -6.383437000 & 0.000000000 \\
\hline 6 & 1.253099000 & 2.855504000 & 0.000000000 & 6 & 0.052333000 & -5.390542000 & 0.000000000 \\
\hline 6 & -1.253243000 & 5.653449000 & 0.000000000 & 6 & 0.951794000 & 5.671055000 & 0.000000000 \\
\hline 6 & -0.424776000 & 6.755032000 & 0.000000000 & 6 & -0.372855000 & 6.033196000 & 0.000000000 \\
\hline 6 & 0.975853000 & 6.589242000 & 0.000000000 & 6 & -1.379474000 & 5.037407000 & 0.000000000 \\
\hline 6 & 1.522652000 & 5.323831000 & 0.000000000 & 6 & -1.043882000 & 3.705256000 & 0.000000000 \\
\hline 1 & -2.712120000 & 0.444582000 & 0.000000000 & 1 & -2.196694000 & 0.651541000 & 0.000000000 \\
\hline 1 & 2.712120000 & -0.444582000 & 0.000000000 & 1 & 3.196921000 & -0.380750000 & 0.000000000 \\
\hline 1 & 2.645493000 & -3.327205000 & 0.000000000 & 1 & 4.138893000 & 2.329428000 & 0.000000000 \\
\hline 1 & -2.332324000 & -2.740269000 & 0.000000000 & 1 & 3.459910000 & 4.705772000 & 0.000000000 \\
\hline 1 & 2.330884000 & -5.781531000 & 0.000000000 & 1 & -3.142714000 & -2.052632000 & 0.000000000 \\
\hline 1 & 0.848539000 & -7.752948000 & 0.000000000 & 1 & 1.722186000 & -3.258236000 & 0.000000000 \\
\hline 1 & -1.621462000 & -7.460154000 & 0.000000000 & 1 & -3.714334000 & -4.461919000 & 0.000000000 \\
\hline 1 & -2.600301000 & -5.195475000 & 0.000000000 & 1 & -3.022557000 & -6.831050000 & 0.000000000 \\
\hline 1 & -2.645493000 & 3.327205000 & 0.000000000 & 1 & -0.607767000 & -7.425636000 & 0.000000000 \\
\hline 1 & 2.332324000 & 2.740269000 & 0.000000000 & 1 & 1.105893000 & -5.650674000 & 0.000000000 \\
\hline 1 & -2.330884000 & 5.781531000 & 0.000000000 & 1 & 1.726938000 & 6.430570000 & 0.000000000 \\
\hline 1 & -0.848539000 & 7.752948000 & 0.000000000 & 1 & -0.652730000 & 7.080469000 & 0.000000000 \\
\hline 1 & 1.621462000 & 7.460154000 & 0.000000000 & 1 & -2.423050000 & 5.331982000 & 0.000000000 \\
\hline 1 & 2.600301000 & 5.195475000 & 0.000000000 & 1 & -1.823789000 & 2.954039000 & 0.000000000 \\
\hline \multicolumn{4}{|c|}{$E=-923.212915281$} & \multicolumn{4}{|c|}{$E=-923.208019913$} \\
\hline \multicolumn{4}{|c|}{ NP5 } & \multicolumn{4}{|c|}{ NP6 } \\
\hline 6 & -2.163873000 & 0.261028000 & 0.000443000 & 6 & -0.749558000 & -1.685802000 & 0.000000000 \\
\hline 6 & -0.086289000 & -0.727431000 & 0.000772000 & 6 & -1.469907000 & 0.497690000 & 0.000000000 \\
\hline 6 & 0.086321000 & 0.727394000 & 0.000947000 & 6 & 0.000000000 & 0.491770000 & 0.000000000 \\
\hline 6 & -1.132481000 & 1.322649000 & 0.000844000 & 6 & 0.431886000 & -0.795988000 & 0.000000000 \\
\hline 6 & 1.132515000 & -1.322673000 & 0.000067000 & 6 & -1.913321000 & 1.776915000 & 0.000000000 \\
\hline 6 & 2.163908000 & -0.261061000 & 0.000426000 & 6 & -0.733473000 & 2.669516000 & 0.000000000 \\
\hline 6 & 1.526666000 & 0.993951000 & 0.000607000 & 6 & 0.439699000 & 1.891583000 & 0.000000000 \\
\hline 6 & -1.526651000 & -0.993942000 & 0.000746000 & 6 & -1.914057000 & -0.897436000 & 0.000000000 \\
\hline
\end{tabular}




\begin{tabular}{|c|c|c|c|c|c|c|c|}
\hline 6 & 3.573541000 & -0.361390000 & -0.000179000 & 6 & -0.674386000 & 4.070785000 & 0.000000000 \\
\hline 6 & 4.329808000 & 0.864587000 & -0.000191000 & 6 & 0.560304000 & 4.692126000 & 0.000000000 \\
\hline 6 & 3.652225000 & 2.109089000 & -0.000143000 & 6 & 1.762814000 & 3.943431000 & 0.000000000 \\
\hline 6 & 2.271457000 & 2.180794000 & -0.000049000 & 6 & 1.711848000 & 2.503390000 & 0.000000000 \\
\hline 6 & -3.573579000 & 0.361390000 & -0.000034000 & 6 & -0.825670000 & -3.097407000 & 0.000000000 \\
\hline 6 & -4.329847000 & -0.864545000 & -0.000135000 & 6 & -2.136644000 & -3.694140000 & 0.000000000 \\
\hline 6 & -3.652212000 & -2.109085000 & 0.000204000 & 6 & -3.286694000 & -2.866021000 & 0.000000000 \\
\hline 6 & -2.271490000 & -2.180811000 & 0.000654000 & 6 & -3.185221000 & -1.487266000 & 0.000000000 \\
\hline 6 & -4.278604000 & 1.597007000 & -0.000266000 & 6 & 3.033743000 & 4.580982000 & 0.000000000 \\
\hline 6 & -5.651202000 & 1.626080000 & -0.000697000 & 6 & 4.194856000 & 3.849480000 & 0.000000000 \\
\hline 6 & -6.396181000 & 0.420783000 & -0.000880000 & 6 & 4.143497000 & 2.432937000 & 0.000000000 \\
\hline 6 & -5.749077000 & -0.789983000 & -0.000592000 & 6 & 2.936515000 & 1.779582000 & 0.000000000 \\
\hline 1 & -1.341534000 & 2.384129000 & 0.000900000 & 6 & 0.311865000 & -3.951328000 & 0.000000000 \\
\hline 1 & 1.341534000 & -2.384166000 & -0.000084000 & 6 & 0.169026000 & -5.316845000 & 0.000000000 \\
\hline 1 & -4.242028000 & -3.019576000 & 0.000057000 & 6 & -1.119986000 & -5.905036000 & 0.000000000 \\
\hline 1 & -1.773962000 & -3.144252000 & 0.000821000 & 6 & -2.240322000 & -5.111547000 & 0.000000000 \\
\hline 1 & -3.720304000 & 2.526024000 & -0.000118000 & 1 & 1.454702000 & -1.146197000 & 0.000000000 \\
\hline 1 & -6.172553000 & 2.576796000 & -0.000909000 & 1 & -2.938099000 & 2.126289000 & 0.000000000 \\
\hline 1 & -7.479540000 & 0.460023000 & -0.001214000 & 1 & -1.585195000 & 4.659828000 & 0.000000000 \\
\hline 1 & -6.317470000 & -1.714378000 & -0.000698000 & 1 & 0.623797000 & 5.775037000 & 0.000000000 \\
\hline 6 & 5.749096000 & 0.790001000 & -0.000135000 & 1 & -4.263802000 & -3.337210000 & 0.000000000 \\
\hline 6 & 6.396182000 & -0.420709000 & -0.000335000 & 1 & -4.078811000 & -0.873086000 & 0.000000000 \\
\hline 6 & 5.651169000 & -1.626068000 & -0.000769000 & 1 & 3.069922000 & 5.665540000 & 0.000000000 \\
\hline 6 & 4.278606000 & -1.597064000 & -0.000689000 & 1 & 5.155781000 & 4.351316000 & 0.000000000 \\
\hline 1 & 7.479531000 & -0.460050000 & 0.000062000 & 1 & 5.066989000 & 1.864703000 & 0.000000000 \\
\hline 1 & 6.172630000 & -2.576720000 & -0.001284000 & 1 & 2.908543000 & 0.697037000 & 0.000000000 \\
\hline 1 & 3.720280000 & -2.526069000 & -0.000905000 & 1 & 1.3035 & -3.513938000 & 0.000000000 \\
\hline 1 & 6.317435000 & 1.714440000 & 0.000216000 & 1 & 1.047032000 & -5.953053000 & 0.000000000 \\
\hline 1 & 4.241992000 & 3.019602000 & -0.000422000 & 1 & -1.216687000 & -6.984785000 & 0.000000000 \\
\hline 1 & 1.773938000 & 3.144235000 & -0.000115000 & 1 & -3.228601000 & -5.559722000 & 0.000000000 \\
\hline \multicolumn{4}{|c|}{$E=-923.201250067$} & \multicolumn{4}{|c|}{$E=-923.201031144$} \\
\hline \multicolumn{4}{|c|}{ NP7 } & \multicolumn{4}{|c|}{$\mathrm{BC} 5$} \\
\hline 6 & 1.505074000 & 1.570864000 & 0.000329000 & 6 & -1.961133000 & 3.646316000 & -0.000466000 \\
\hline 6 & 0.533151000 & -0.509583000 & -0.000530000 & 6 & -1.501226000 & 2.277622000 & -0.004169000 \\
\hline 6 & -0.533201000 & 0.509481000 & -0.000481000 & 6 & -2.409741000 & 1.279048000 & 0.003583000 \\
\hline 6 & 0.036443000 & 1.739732000 & 0.000243000 & 6 & -0.096840000 & 2.725784000 & 0.003686000 \\
\hline 6 & -0.036509000 & -1.739824000 & 0.000002000 & 6 & -3.815644000 & 1.608143000 & -0.000626000 \\
\hline 6 & -1.505146000 & -1.570945000 & 0.000113000 & 6 & -2.723776000 & -0.161024000 & -0.004170000 \\
\hline 6 & -1.816999000 & -0.199491000 & -0.000156000 & 6 & -4.138994000 & 0.124752000 & -0.000423000 \\
\hline 6 & 1.816961000 & 0.199412000 & -0.000007000 & 6 & -2.312870000 & -1.447005000 & 0.004788000 \\
\hline 6 & -2.514737000 & -2.545031000 & 0.000525000 & 6 & -3.300324000 & -2.500301000 & 0.001017000 \\
\hline 6 & -3.836812000 & -2.142853000 & 0.000603000 & 6 & -1.222226000 & -2.438645000 & -0.002764000 \\
\hline 6 & -4.190446000 & -0.770921000 & 0.000239000 & 6 & -2.177275000 & -3.521646000 & 0.000756000 \\
\hline 6 & -3.159823000 & 0.236109000 & -0.000205000 & 6 & 0.096828000 & -2.725770000 & 0.003349000 \\
\hline 6 & 2.514629000 & 2.544996000 & 0.000628000 & 6 & 0.515165000 & -4.107863000 & -0.000294000 \\
\hline 6 & 3.836733000 & 2.142881000 & 0.000489000 & 6 & 1.501222000 & -2.277614000 & -0.003947000 \\
\hline 6 & -5.550190000 & -0.355965000 & 0.000296000 & 6 & 1.961124000 & -3.646293000 & -0.000608000 \\
\hline 6 & -5.886074000 & 0.974712000 & -0.000088000 & 6 & 2.409787000 & -1.279067000 & 0.003641000 \\
\hline 6 & -4.871497000 & 1.964493000 & -0.000599000 & 6 & 3.815659000 & -1.608139000 & -0.000393000 \\
\hline
\end{tabular}




\begin{tabular}{|c|c|c|c|c|c|c|c|}
\hline 6 & -3.546825000 & 1.604982000 & -0.000665000 & 6 & 2.723752000 & 0.161020000 & -0.003880000 \\
\hline 1 & -0.453604000 & 2.704291000 & 0.000812000 & 6 & 4.139004000 & -0.124738000 & -0.000247000 \\
\hline 1 & 0.453529000 & -2.704391000 & 0.000441000 & 6 & 2.312859000 & 1.446989000 & 0.005023000 \\
\hline 1 & -2.261849000 & -3.599837000 & 0.000798000 & 6 & 3.300320000 & 2.500303000 & 0.000976000 \\
\hline 1 & -4.630099000 & -2.882730000 & 0.000944000 & 6 & 1.222220000 & 2.438640000 & -0.002825000 \\
\hline 1 & -6.324493000 & -1.116223000 & 0.000644000 & 6 & 2.177261000 & 3.521626000 & 0.000933000 \\
\hline 1 & -6.927891000 & 1.274463000 & -0.000031000 & 6 & -0.515162000 & 4.107862000 & -0.000376000 \\
\hline 1 & -5.147907000 & 3.012988000 & -0.000958000 & 6 & -3.294690000 & 3.973451000 & -0.000381000 \\
\hline 1 & -2.780283000 & 2.369857000 & -0.001123000 & 6 & -4.266888000 & 2.904956000 & -0.000627000 \\
\hline 6 & 4.190453000 & 0.770947000 & 0.000092000 & 6 & -4.649104000 & -2.243064000 & 0.000247000 \\
\hline 6 & 3.159833000 & -0.236104000 & -0.000266000 & 6 & -5.088777000 & -0.866950000 & -0.000080000 \\
\hline 6 & 5.550195000 & 0.356064000 & 0.000141000 & 6 & -0.381930000 & -5.147348000 & -0.000182000 \\
\hline 6 & 5.886169000 & -0.974606000 & -0.000094000 & 6 & -1.793461000 & -4.840003000 & 0.000425000 \\
\hline 6 & 4.871644000 & -1.964416000 & -0.000440000 & 6 & 4.266893000 & -2.904977000 & -0.000680000 \\
\hline 6 & 3.546958000 & -1.604973000 & -0.000525000 & 6 & 3.294693000 & -3.973447000 & -0.000554000 \\
\hline 1 & 6.324488000 & 1.116329000 & 0.000380000 & 6 & 4.649090000 & 2.243083000 & 0.000065000 \\
\hline 1 & 6.928017000 & -1.274247000 & -0.000010000 & 6 & 5.088776000 & 0.866952000 & -0.000203000 \\
\hline 1 & 5.148057000 & -3.012913000 & -0.000632000 & 6 & 0.381948000 & 5.147353000 & -0.000302000 \\
\hline 1 & 2.780469000 & -2.369901000 & -0.000847000 & 6 & 1.793460000 & 4.839998000 & 0.000365000 \\
\hline 1 & 4.630003000 & 2.882778000 & 0.000723000 & 1 & -3.640091000 & 5.000730000 & 0.000862000 \\
\hline 1 & 2.261647000 & 3.599779000 & 0.000996000 & 1 & -5.322081000 & 3.152241000 & -0.001953000 \\
\hline & & & & 1 & -5.390532000 & -3.033573000 & -0.001174000 \\
\hline & -923.200888035 & & & 1 & -6.151184000 & -0.652657000 & 0.000966000 \\
\hline & & & & 1 & -0.068145000 & -6.184703000 & -0.001445000 \\
\hline & & & & 1 & -2.510195000 & -5.652959000 & 0.001494000 \\
\hline & & & & 1 & 5.322081000 & -3.152282000 & -0.002065000 \\
\hline & & & & 1 & 3.640076000 & -5.000731000 & 0.000532000 \\
\hline & & & & 1 & 5.390512000 & 3.033595000 & -0.001573000 \\
\hline & & & & 1 & 6.151181000 & 0.652663000 & 0.000760000 \\
\hline & & & & 1 & 0.068164000 & 6.184707000 & -0.001767000 \\
\hline & & & & 1 & 2.510209000 & 5.652941000 & 0.001412000 \\
\hline & & & & & $13 / 9.0050659 /$ & & \\
\hline & & & & & & & \\
\hline 6 & 0.771717000 & 3.272258000 & -0.000007000 & 6 & 0.584163000 & -8.407683000 & -0.000053000 \\
\hline 6 & 0.752246000 & 1.881887000 & -0.000197000 & 6 & 0.605196000 & -6.961929000 & -0.000086000 \\
\hline 6 & 1.906249000 & 1.100573000 & -0.000137000 & 6 & -0.927158000 & -8.376772000 & -0.000024000 \\
\hline 6 & -0.752246000 & 1.881887000 & -0.000197000 & 6 & -0.889125000 & -6.931374000 & -0.000057000 \\
\hline 6 & 3.174486000 & 1.832790000 & -0.000037000 & 6 & 1.740775000 & -9.130331000 & 0.000006000 \\
\hline 6 & 2.005885000 & -0.289479000 & -0.000197000 & 6 & 2.983261000 & -8.393169000 & 0.000013000 \\
\hline 6 & -0.771717000 & 3.272258000 & -0.000007000 & 6 & 3.001818000 & -6.954444000 & -0.000018000 \\
\hline 6 & -1.906249000 & 1.100573000 & -0.000137000 & 6 & 4.269721000 & -6.256568000 & 0.000004000 \\
\hline 6 & -3.174486000 & 1.832790000 & -0.000037000 & 6 & 5.495329000 & -7.010350000 & 0.000044000 \\
\hline 6 & -2.005885000 & -0.289479000 & -0.000197000 & 6 & 1.754287000 & -6.233308000 & -0.000063000 \\
\hline 6 & -3.219717000 & -0.967803000 & -0.000007000 & 6 & 4.223880000 & -9.097190000 & 0.000055000 \\
\hline 6 & -1.253639000 & -1.592408000 & -0.000197000 & 6 & 4.327704000 & -4.816722000 & -0.000010000 \\
\hline 6 & -2.448000000 & -2.304455000 & -0.000007000 & 6 & 5.557982000 & -4.235544000 & 0.000012000 \\
\hline 6 & 0.000000000 & -2.201147000 & -0.000137000 & 6 & 6.790756000 & -4.991074000 & 0.000047000 \\
\hline 6 & 0.000000000 & -3.665581000 & -0.000037000 & 6 & 6.331638000 & -2.956650000 & 0.000030000 \\
\hline 6 & 1.253639000 & -1.592408000 & -0.000197000 & 6 & 7.573144000 & -3.697796000 & 0.000066000 \\
\hline
\end{tabular}




\begin{tabular}{|c|c|c|c|c|c|c|c|}
\hline 6 & 2.448000000 & -2.304455000 & -0.000007000 & 6 & 6.782727000 & -6.354835000 & 0.000065000 \\
\hline 6 & 3.219717000 & -0.967803000 & -0.000007000 & 6 & 5.426670000 & -8.435155000 & 0.000068000 \\
\hline 6 & 1.963718000 & 3.993890000 & 0.000110000 & 6 & -2.112350000 & -9.051497000 & -0.000010000 \\
\hline 6 & -1.963718000 & 3.993890000 & 0.000110000 & 6 & -3.323659000 & -8.264149000 & -0.000016000 \\
\hline 6 & -4.440669000 & -0.296315000 & 0.000110000 & 6 & -3.283488000 & -6.825808000 & -0.000031000 \\
\hline 6 & -2.476951000 & -3.697575000 & 0.000110000 & 6 & -4.521911000 & -6.076775000 & -0.000020000 \\
\hline 6 & 2.476951000 & -3.697575000 & 0.000110000 & 6 & -5.777259000 & -6.779941000 & -0.000032000 \\
\hline 6 & 4.440669000 & -0.296315000 & 0.000110000 & 6 & -2.007405000 & -6.156325000 & -0.000064000 \\
\hline 6 & 3.149286000 & 3.254211000 & 0.000089000 & 6 & 6.275344000 & -1.597172000 & 0.000012000 \\
\hline 6 & 4.392872000 & 1.100256000 & 0.000089000 & 6 & 7.523808000 & -0.877569000 & 0.000036000 \\
\hline 6 & -3.149286000 & 3.254211000 & 0.000089000 & 6 & 8.760451000 & -1.613132000 & 0.000073000 \\
\hline 6 & -4.392872000 & 1.100256000 & 0.000089000 & 6 & 7.553400000 & 0.569373000 & 0.000026000 \\
\hline 6 & -1.243586000 & -4.354467000 & 0.000089000 & 6 & 8.819106000 & 1.253663000 & 0.000058000 \\
\hline 6 & 1.243586000 & -4.354467000 & 0.000089000 & 6 & 8.777384000 & -3.057686000 & 0.000085000 \\
\hline 1 & 1.993808000 & 5.077077000 & 0.000308000 & 6 & 9.990574000 & -0.890926000 & 0.000100000 \\
\hline 1 & 4.098679000 & 3.779288000 & 0.000237000 & 6 & 6.335516000 & 1.339479000 & -0.000013000 \\
\hline 1 & -1.993808000 & 5.077077000 & 0.000308000 & 6 & 6.447485000 & 2.695527000 & -0.000020000 \\
\hline 1 & -4.098679000 & 3.779288000 & 0.000237000 & 6 & 7.718313000 & 3.385268000 & 0.000014000 \\
\hline 1 & -5.322299000 & 1.659916000 & 0.000237000 & 6 & 5.726812000 & 4.004964000 & -0.000012000 \\
\hline 1 & -5.393782000 & -0.811850000 & 0.000308000 & 6 & 6.989414000 & 4.709544000 & 0.000021000 \\
\hline 1 & -3.399974000 & -4.265227000 & 0.000308000 & 6 & 8.895298000 & 2.696324000 & 0.000057000 \\
\hline 1 & -1.223620000 & -5.439204000 & 0.000237000 & 6 & 10.018599000 & 0.481727000 & 0.000094000 \\
\hline 1 & 1.223620000 & -5.439204000 & 0.000237000 & 6 & 4.521269000 & 4.635799000 & -0.000018000 \\
\hline 1 & 3.399974000 & -4.265227000 & 0.000308000 & 6 & 4.521911000 & 6.076776000 & 0.000011000 \\
\hline 1 & 5.393782000 & -0.811850000 & 0.000308000 & 6 & 5.777259000 & 6.779941000 & 0.000027000 \\
\hline \multirow[t]{2}{*}{1} & 5.322299000 & 1.659916000 & 0.000237000 & 6 & 3.283489000 & 6.825809000 & 0.000019000 \\
\hline & & & & 6 & 3.323660000 & 8.264149000 & 0.000008000 \\
\hline & 1150.49399789 & & & 6 & 7.036891000 & 6.072510000 & 0.000037000 \\
\hline & & & & 6 & -4.592003000 & -8.916907000 & -0.000015000 \\
\hline & & & & 6 & -4.521269000 & -4.635799000 & 0.000007000 \\
\hline & & & & 6 & -5.726812000 & -4.004964000 & 0.000004000 \\
\hline & & & & 6 & -6.989414000 & -4.709544000 & -0.000024000 \\
\hline & & & & 6 & -6.447485000 & -2.695526000 & 0.000016000 \\
\hline & & & & 6 & -7.718314000 & -3.385268000 & -0.000013000 \\
\hline & & & & 6 & -7.036891000 & -6.072509000 & -0.000039000 \\
\hline & & & & 6 & -5.766740000 & -8.206321000 & -0.000030000 \\
\hline & & & & 6 & -8.895298000 & -2.696324000 & -0.000048000 \\
\hline & & & & 6 & -8.819106000 & -1.253663000 & -0.000047000 \\
\hline & & & & 6 & -7.553400000 & -0.569373000 & -0.000022000 \\
\hline & & & & 6 & -7.523808000 & 0.877569000 & -0.000030000 \\
\hline & & & & 6 & -8.760452000 & 1.613132000 & -0.000058000 \\
\hline & & & & 6 & -6.335516000 & -1.339478000 & 0.000009000 \\
\hline & & & & 6 & -10.018599000 & -0.481727000 & -0.00007500 \\
\hline & & & & 6 & -6.275344000 & 1.597171000 & -0.000012000 \\
\hline & & & & 6 & -6.331639000 & 2.956650000 & -0.000027000 \\
\hline & & & & 6 & -7.573144000 & 3.697796000 & -0.000055000 \\
\hline & & & & 6 & -5.557982000 & 4.235543000 & -0.000012000 \\
\hline & & & & 6 & -6.790756000 & 4.991073000 & -0.000039000 \\
\hline & & & & 6 & -8.777384000 & 3.057686000 & -0.000067000 \\
\hline & & & & 6 & -9.990574000 & 0.890926000 & -0.000079000 \\
\hline & & & & 6 & -6.782727000 & 6.354835000 & -0.000052000 \\
\hline
\end{tabular}




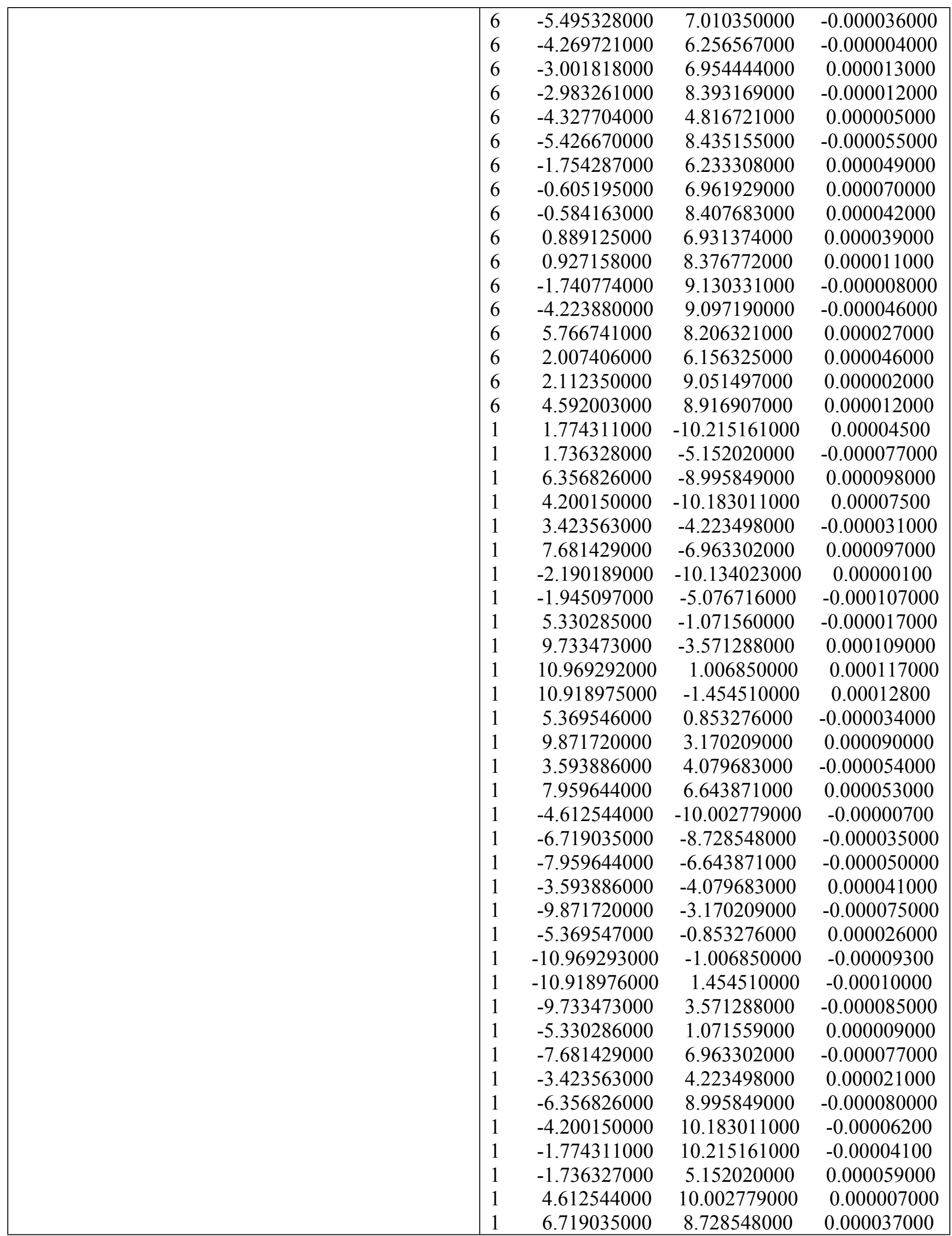




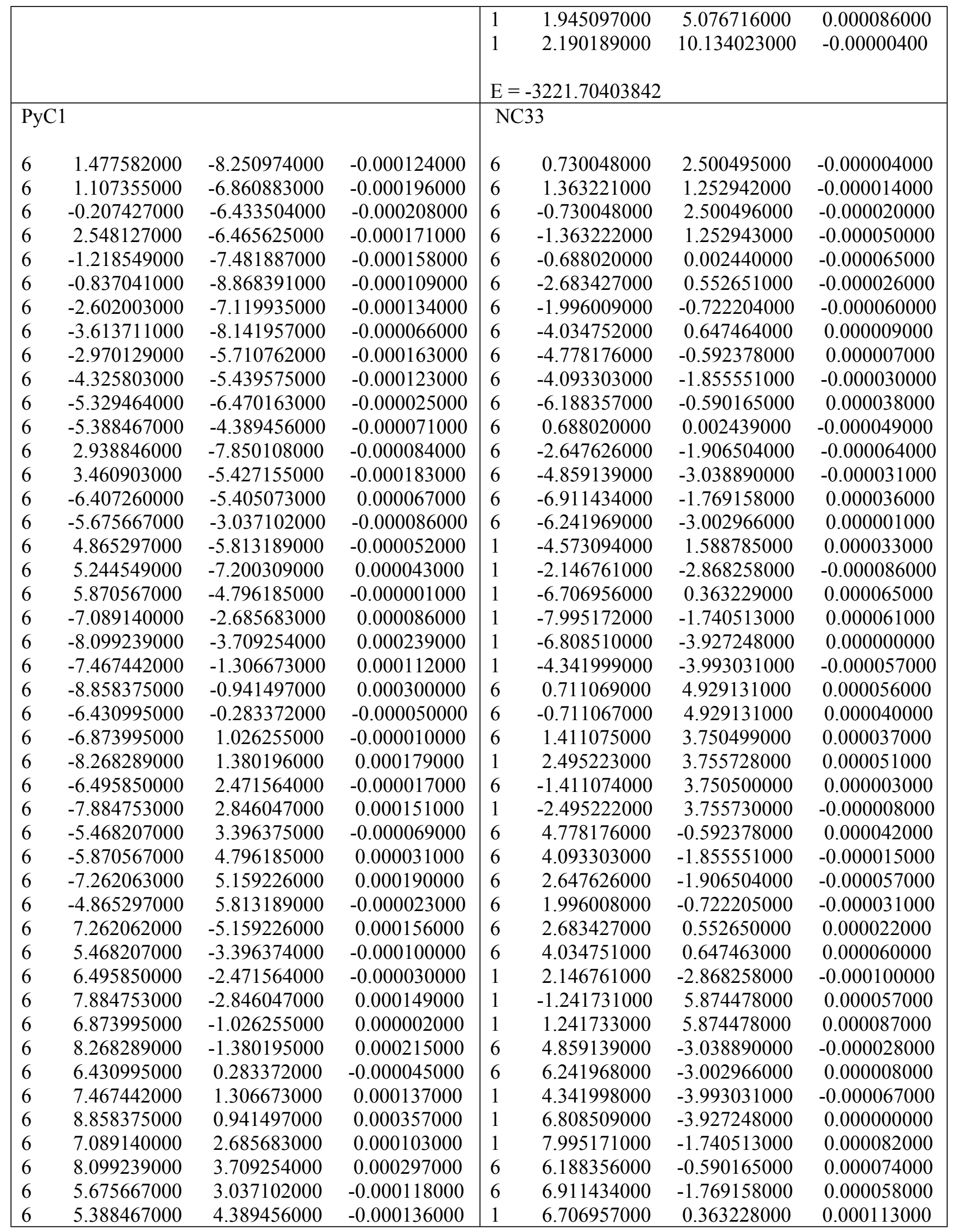




\begin{tabular}{|c|c|c|c|c|c|c|}
\hline 6 & 6.407260000 & 5.405073000 & 0.000067000 & 4.573094000 & 1.588784000 & 0.000095000 \\
\hline 6 & 4.325803000 & 5.439575000 & -0.000188000 & & & \\
\hline 6 & 5.329464000 & 6.470163000 & -0.000023000 & $E=-1153.01851291$ & & \\
\hline 6 & 2.970129000 & 5.710762000 & -0.000266000 & & & \\
\hline 6 & 2.602003000 & 7.119935000 & -0.000158000 & & & \\
\hline 6 & 3.613711000 & 8.141957000 & -0.000011000 & & & \\
\hline 6 & 1.218549000 & 7.481887000 & -0.000180000 & & & \\
\hline 6 & 0.837041000 & 8.868391000 & -0.000055000 & & & \\
\hline 6 & 0.207427000 & 6.433504000 & -0.000304000 & & & \\
\hline 6 & -1.107355000 & 6.860883000 & -0.000245000 & & & \\
\hline 6 & -1.477582000 & 8.250974000 & -0.000118000 & & & \\
\hline 6 & -2.548127000 & 6.465625000 & -0.000212000 & & & \\
\hline 6 & -2.938846000 & 7.850107000 & -0.000075000 & & & \\
\hline 6 & -3.460903000 & 5.427155000 & -0.000179000 & & & \\
\hline 6 & -5.244549000 & 7.200309000 & 0.000092000 & & & \\
\hline 6 & 0.550613000 & -9.254593000 & -0.000090000 & & & \\
\hline 6 & -1.866749000 & -9.852893000 & -0.000058000 & & & \\
\hline 6 & -5.012735000 & -7.799113000 & -0.000002000 & & & \\
\hline 6 & -3.198108000 & -9.504570000 & -0.000037000 & & & \\
\hline 6 & 4.248078000 & -8.240401000 & 0.000015000 & & & \\
\hline 6 & -7.739903000 & -5.104100000 & 0.000223000 & & & \\
\hline 6 & -9.466702000 & -3.309802000 & 0.000410000 & & & \\
\hline 6 & -9.260928000 & 0.441522000 & 0.000343000 & & & \\
\hline 6 & -9.830765000 & -1.982665000 & 0.000441000 & & & \\
\hline 6 & -8.290433000 & 4.150617000 & 0.000257000 & & & \\
\hline 6 & 6.632364000 & -7.521919000 & 0.000181000 & & & \\
\hline 6 & 8.290433000 & -4.150617000 & 0.000241000 & & & \\
\hline 6 & 7.599835000 & -6.543217000 & 0.000238000 & & & \\
\hline 6 & 9.260928000 & -0.441522000 & 0.000399000 & & & \\
\hline 6 & 9.830765000 & 1.982665000 & 0.000530000 & & & \\
\hline 6 & 7.739903000 & 5.104100000 & 0.000289000 & & & \\
\hline 6 & 9.466702000 & 3.309802000 & 0.000503000 & & & \\
\hline 6 & 5.012735000 & 7.799113000 & 0.000061000 & & & \\
\hline 6 & 3.198108000 & 9.504570000 & 0.000089000 & & & \\
\hline 6 & -0.550613000 & 9.254593000 & -0.000030000 & & & \\
\hline 6 & 1.866748000 & 9.852893000 & 0.000067000 & & & \\
\hline 6 & -7.599835000 & 6.543218000 & 0.000288000 & & & \\
\hline 6 & -4.248078000 & 8.240401000 & 0.000072000 & & & \\
\hline 6 & -6.632365000 & 7.521919000 & 0.000243000 & & & \\
\hline 6 & -4.068866000 & 3.065153000 & -0.000213000 & & & \\
\hline 6 & -3.113526000 & 4.031613000 & -0.000259000 & & & \\
\hline 6 & 4.068866000 & -3.065153000 & -0.000274000 & & & \\
\hline 6 & 3.113526000 & -4.031613000 & -0.000307000 & & & \\
\hline 6 & -0.620594000 & -5.056075000 & -0.000234000 & & & \\
\hline 6 & -1.935258000 & -4.712147000 & -0.000217000 & & & \\
\hline 6 & -4.689241000 & -1.990658000 & -0.000258000 & & & \\
\hline 6 & -5.048734000 & -0.680144000 & -0.000245000 & & & \\
\hline 6 & 0.620594000 & 5.056075000 & -0.000476000 & & & \\
\hline 6 & 1.935258000 & 4.712147000 & -0.000462000 & & & \\
\hline 6 & 4.689241000 & 1.990658000 & -0.000284000 & & & \\
\hline 6 & 5.048734000 & 0.680144000 & -0.000252000 & & & \\
\hline
\end{tabular}




\begin{tabular}{|c|c|c|c|c|c|c|c|}
\hline 1 & 0.807967000 & -10.308685000 & -0.00003000 & & & & \\
\hline 1 & -3.961337000 & -10.277201000 & 0.00001100 & & & & \\
\hline 1 & -1.579701000 & -10.900316000 & -0.00002500 & & & & \\
\hline 1 & -5.753316000 & -8.592135000 & 0.000077000 & & & & \\
\hline 1 & 4.564647000 & -9.278259000 & 0.000091000 & & & & \\
\hline 1 & -8.524265000 & -5.853869000 & 0.000330000 & & & & \\
\hline 1 & -10.881488000 & -1.707969000 & 0.00057300 & & & & \\
\hline 1 & -10.230333000 & -4.082069000 & 0.00051700 & & & & \\
\hline 1 & -10.317964000 & 0.686390000 & 0.00048800 & & & & \\
\hline 1 & -9.331918000 & 4.454902000 & 0.000390000 & & & & \\
\hline 1 & 6.919765000 & -8.569242000 & 0.000250000 & & & & \\
\hline 1 & 8.650449000 & -6.818355000 & 0.000350000 & & & & \\
\hline 1 & 9.331918000 & -4.454902000 & 0.000381000 & & & & \\
\hline 1 & 10.317964000 & -0.686390000 & 0.00055900 & & & & \\
\hline 1 & 10.881488000 & 1.707969000 & 0.000683000 & & & & \\
\hline 1 & 10.230333000 & 4.082069000 & 0.000638000 & & & & \\
\hline 1 & 8.524265000 & 5.853869000 & 0.000446000 & & & & \\
\hline 1 & 5.753316000 & 8.592135000 & 0.000198000 & & & & \\
\hline 1 & 3.961337000 & 10.277201000 & 0.000196000 & & & & \\
\hline 1 & 1.579701000 & 10.900316000 & 0.000158000 & & & & \\
\hline 1 & -0.807966000 & 10.308685000 & 0.00008000 & & & & \\
\hline 1 & -6.919765000 & 8.569242000 & 0.000327000 & & & & \\
\hline 1 & -8.650449000 & 6.818355000 & 0.000403000 & & & & \\
\hline 1 & -4.564646000 & 9.278259000 & 0.000184000 & & & & \\
\hline 1 & 3.783798000 & -2.020283000 & -0.000383000 & & & & \\
\hline 1 & 2.065469000 & -3.758591000 & -0.000444000 & & & & \\
\hline 1 & 0.141458000 & -4.286475000 & -0.000255000 & & & & \\
\hline 1 & -2.222720000 & -3.667958000 & -0.000224000 & & & & \\
\hline 1 & -0.141458000 & 4.286475000 & -0.000642000 & & & & \\
\hline 1 & 2.222720000 & 3.667958000 & -0.000612000 & & & & \\
\hline 1 & 3.641799000 & 2.265993000 & -0.000440000 & & & & \\
\hline 1 & 4.288284000 & -0.091059000 & -0.000389000 & & & & \\
\hline 1 & -3.641798000 & -2.265993000 & -0.000422000 & & & & \\
\hline 1 & -4.288284000 & 0.091059000 & -0.000397000 & & & & \\
\hline 1 & -3.783798000 & 2.020283000 & -0.000277000 & & & & \\
\hline 1 & -2.065469000 & 3.758591000 & -0.000345000 & & & & \\
\hline \multicolumn{8}{|c|}{$E=-3678.98165485$} \\
\hline \multicolumn{4}{|c|}{ NC34 } & \multicolumn{4}{|c|}{ NC35 } \\
\hline 6 & -0.462928000 & 2.710741000 & 0.000016000 & 6 & 1.139661000 & 1.912291000 & -0.000071000 \\
\hline 6 & -1.059289000 & 1.454896000 & -0.000026000 & 6 & 1.510703000 & 0.570549000 & -0.000142000 \\
\hline 6 & 1.005128000 & 2.747534000 & -0.000029000 & 6 & -0.300917000 & 2.205177000 & -0.000176000 \\
\hline 6 & 1.664981000 & 1.526487000 & -0.000114000 & 6 & -1.167040000 & 1.118033000 & -0.000381000 \\
\hline 6 & 1.024818000 & 0.253214000 & -0.000167000 & 6 & -0.747362000 & -0.251372000 & -0.000479000 \\
\hline 6 & 3.006952000 & 0.836922000 & -0.000133000 & 6 & -2.594072000 & 0.687662000 & -0.000230000 \\
\hline 6 & 2.378190000 & -0.415096000 & -0.000168000 & 6 & -2.173073000 & -0.697785000 & -0.000286000 \\
\hline 6 & 4.390832000 & 0.975484000 & 0.000014000 & 6 & -3.902904000 & 1.046307000 & -0.000059000 \\
\hline 6 & 5.129042000 & -0.207423000 & 0.000096000 & 6 & -4.874094000 & -0.020728000 & 0.000059000 \\
\hline 6 & 4.521753000 & -1.486916000 & 0.000059000 & 6 & -4.452496000 & -1.395400000 & 0.000006000 \\
\hline 6 & -0.341164000 & 0.213482000 & -0.000090000 & 6 & -6.257698000 & 0.259376000 & 0.000226000 \\
\hline
\end{tabular}




\begin{tabular}{|c|c|c|c|c|c|c|c|}
\hline 6 & 3.077963000 & -1.619601000 & -0.000064000 & 6 & 0.591336000 & -0.518650000 & -0.000237000 \\
\hline 1 & 4.887272000 & 1.938380000 & 0.000060000 & 6 & -3.047521000 & -1.730452000 & -0.000176000 \\
\hline 6 & -0.502995000 & 5.141226000 & 0.000136000 & 6 & -5.438246000 & -2.404501000 & 0.000125000 \\
\hline 6 & 0.924398000 & 5.177590000 & 0.000102000 & 6 & -7.198305000 & -0.753000000 & 0.000337000 \\
\hline 6 & -1.173028000 & 3.949319000 & 0.000093000 & 6 & -6.785712000 & -2.095993000 & 0.000286000 \\
\hline 1 & -2.257075000 & 3.928276000 & 0.000118000 & 1 & -4.244886000 & 2.075395000 & -0.000004000 \\
\hline 6 & 1.653480000 & 4.021723000 & 0.000022000 & 1 & -2.746073000 & -2.772181000 & -0.000218000 \\
\hline 1 & 2.737264000 & 4.055364000 & -0.000008000 & 1 & -6.577658000 & 1.296455000 & 0.000266000 \\
\hline 6 & -4.415474000 & -0.487601000 & 0.000015000 & 1 & -8.255185000 & -0.511351000 & 0.000462000 \\
\hline 6 & -3.696078000 & -1.732340000 & -0.000045000 & 1 & -7.524118000 & -2.889744000 & 0.000374000 \\
\hline 6 & -2.251884000 & -1.743541000 & -0.000093000 & 1 & -5.119669000 & -3.442023000 & 0.000086000 \\
\hline 6 & -1.630684000 & -0.541253000 & -0.000080000 & 6 & 1.590765000 & 4.305350000 & 0.000210000 \\
\hline 6 & -2.352182000 & 0.713844000 & -0.000014000 & 6 & 0.192035000 & 4.586122000 & 0.000144000 \\
\hline 6 & -3.707980000 & 0.769871000 & 0.000030000 & 6 & 2.046638000 & 3.016671000 & 0.000103000 \\
\hline 1 & -1.726405000 & -2.692210000 & -0.000139000 & 1 & 3.111382000 & 2.819070000 & 0.000153000 \\
\hline 1 & 1.428636000 & 6.137328000 & 0.000138000 & 6 & -0.721605000 & 3.569255000 & -0.000047000 \\
\hline 1 & -1.055397000 & 6.074068000 & 0.000196000 & 1 & -1.784247000 & 3.784735000 & -0.000115000 \\
\hline 6 & -4.429986000 & -2.937061000 & -0.000056000 & 6 & 4.456366000 & -2.014104000 & 0.000042000 \\
\hline 6 & -5.812235000 & -2.938797000 & -0.000010000 & 6 & 3.503128000 & -3.060322000 & -0.000089000 \\
\hline 1 & -3.886632000 & -3.876565000 & -0.000102000 & 6 & 2.129640000 & -2.820062000 & -0.000213000 \\
\hline 1 & -6.353725000 & -3.877967000 & -0.000019000 & 6 & 1.743797000 & -1.488050000 & -0.000205000 \\
\hline 1 & -7.599999000 & -1.724451000 & 0.000085000 & 6 & 2.654877000 & -0.422472000 & -0.000062000 \\
\hline 6 & -5.826570000 & -0.524934000 & 0.000062000 & 6 & 4.039452000 & -0.624026000 & 0.000057000 \\
\hline 6 & -6.515874000 & -1.722653000 & 0.000049000 & 1 & 1.418846000 & -3.637539000 & -0.000312000 \\
\hline 1 & -6.370968000 & 0.413932000 & 0.000108000 & 6 & 5.852438000 & -2.294754000 & 0.000152000 \\
\hline 1 & -4.271865000 & 1.696138000 & 0.000079000 & 6 & 6.781670000 & -1.286671000 & 0.000269000 \\
\hline 6 & 2.494359000 & -2.918549000 & -0.000079000 & 1 & 6.171829000 & -3.331874000 & 40000 \\
\hline 6 & 4.703921000 & -3.915008000 & 0.000136000 & 1 & 7.839773000 & -1.522413000 & 0.000351000 \\
\hline 6 & 3.288037000 & -4.035456000 & 0.000020000 & 1 & 7.114590000 & 0.857094000 & 0.000375000 \\
\hline 1 & 5.314199000 & -4.810983000 & 0.000210000 & 6 & 5.035189000 & 0.393286000 & 0.000180000 \\
\hline 1 & 2.838086000 & -5.021886000 & 0.000008000 & 6 & 6.367520000 & 0.071360000 & 0.000283000 \\
\hline 1 & 1.413896000 & -3.007619000 & -0.000172000 & 1 & 4.727688000 & 1.431574000 & 0.000192000 \\
\hline 6 & 5.299273000 & -2.679169000 & 0.000153000 & 1 & -0.142129000 & 5.617428000 & 37000 \\
\hline 1 & 6.381107000 & -2.594034000 & 0.000239000 & 1 & 2.296229000 & 5.128583000 & 0.000347000 \\
\hline 1 & 6.212961000 & -0.159377000 & 0.000198000 & 1 & 3.868607000 & -4.081804000 & -0.000093000 \\
\hline \multicolumn{4}{|c|}{$E=-1153.00724998$} & \multicolumn{4}{|c|}{$E=-1153.00643011$} \\
\hline \multicolumn{4}{|c|}{ NC36 } & \multicolumn{4}{|c|}{ NC37 } \\
\hline 6 & -1.109090000 & 2.073991000 & 0.000002000 & 6 & -0.737636000 & 2.920249000 & -0.000008000 \\
\hline 6 & -1.315797000 & 0.706031000 & -0.000033000 & 6 & -1.358842000 & 1.689199000 & -0.000023000 \\
\hline 6 & 0.295112000 & 2.538614000 & -0.000026000 & 6 & 0.737634000 & 2.920250000 & -0.000009000 \\
\hline 6 & 1.277205000 & 1.570530000 & -0.000087000 & 6 & 1.358841000 & 1.689200000 & -0.000024000 \\
\hline 6 & 1.028893000 & 0.151950000 & -0.000123000 & 6 & 0.680103000 & 0.415897000 & -0.000026000 \\
\hline 6 & 2.750927000 & 1.294747000 & -0.000102000 & 6 & 2.672371000 & 0.974255000 & -0.000010000 \\
\hline 6 & 2.513563000 & -0.087792000 & -0.000134000 & 6 & 2.025508000 & -0.270481000 & -0.000026000 \\
\hline 6 & 4.038653000 & 1.829421000 & 0.000021000 & 6 & 4.060599000 & 1.099216000 & 0.000004000 \\
\hline 6 & 5.085960000 & 0.913864000 & 0.000092000 & 6 & 4.785900000 & -0.086858000 & 0.000005000 \\
\hline 6 & 4.877363000 & -0.489892000 & 0.000064000 & 6 & 4.164688000 & -1.362195000 & -0.000004000 \\
\hline 6 & -0.258044000 & -0.274645000 & -0.000082000 & 6 & -0.680103000 & 0.415897000 & -0.000051000 \\
\hline 6 & 3.536378000 & -1.037146000 & -0.000039000 & 6 & 2.720623000 & -1.482699000 & -0.000017000 \\
\hline
\end{tabular}




\begin{tabular}{|c|c|c|c|c|c|c|c|}
\hline 1 & 4.233219000 & 2.895174000 & 0.000059000 & 1 & 4.563831000 & 2.058570000 & 0.000011000 \\
\hline 6 & -1.838112000 & 4.400255000 & 0.000095000 & 6 & -0.716790000 & 5.352974000 & 0.000034000 \\
\hline 6 & -0.477205000 & 4.847086000 & 0.000072000 & 6 & 0.716785000 & 5.352975000 & 0.000028000 \\
\hline 6 & -2.139680000 & 3.070397000 & 0.000061000 & 6 & -1.416646000 & 4.182881000 & 0.000017000 \\
\hline 1 & -3.174242000 & 2.750151000 & 0.000079000 & 1 & -2.500999000 & 4.191007000 & 0.000021000 \\
\hline 6 & 0.548501000 & 3.949714000 & 0.000014000 & 6 & 1.416643000 & 4.182883000 & 0.000008000 \\
\hline 1 & 1.577950000 & 4.290607000 & -0.000006000 & 1 & 2.500996000 & 4.191010000 & 0.000005000 \\
\hline 6 & -3.921866000 & -2.210343000 & -0.000034000 & 6 & -4.785899000 & -0.086861000 & 0.000084000 \\
\hline 6 & -2.849242000 & -3.137515000 & -0.000078000 & 6 & -4.164687000 & -1.362198000 & 0.000039000 \\
\hline 6 & -1.516114000 & -2.737051000 & -0.000101000 & 6 & -2.720622000 & -1.482700000 & -0.000025000 \\
\hline 6 & -1.287389000 & -1.366761000 & -0.000077000 & 6 & -2.025508000 & -0.270482000 & -0.000037000 \\
\hline 6 & -2.319400000 & -0.415326000 & -0.000030000 & 6 & -2.672371000 & 0.974253000 & -0.000004000 \\
\hline 6 & -3.672998000 & -0.782467000 & -0.000009000 & 6 & -4.060600000 & 1.099213000 & 0.000065000 \\
\hline 1 & -0.715036000 & -3.466424000 & -0.000137000 & 1 & 1.243532000 & 6.300613000 & 0.000042000 \\
\hline 6 & -5.273306000 & -2.655448000 & -0.000013000 & 1 & -1.243538000 & 6.300612000 & 0.000052000 \\
\hline 6 & -6.316658000 & -1.764415000 & 0.000027000 & 6 & 2.135126000 & -2.778769000 & -0.000012000 \\
\hline 1 & -5.466946000 & -3.723212000 & -0.000031000 & 6 & 4.935739000 & -2.556906000 & 0.000003000 \\
\hline 1 & -7.339098000 & -2.124722000 & 0.000042000 & 6 & 2.921229000 & -3.902312000 & -0.000002000 \\
\hline 1 & -6.901232000 & 0.323889000 & 0.000082000 & 6 & 4.335782000 & -3.791589000 & 0.000003000 \\
\hline 6 & -4.780562000 & 0.109046000 & 0.000033000 & 1 & 1.055839000 & -2.864713000 & -0.000010000 \\
\hline 6 & -6.066710000 & -0.368232000 & 0.000050000 & 1 & 2.463060000 & -4.884996000 & 0.000004000 \\
\hline 1 & -4.598288000 & 1.176372000 & 0.000050000 & 1 & 4.942079000 & -4.690208000 & 0.000009000 \\
\hline 1 & -0.270093000 & 5.911232000 & 0.000099000 & 1 & 6.017937000 & -2.476148000 & 0.000010000 \\
\hline 1 & -2.634927000 & 5.135495000 & 0.000140000 & 1 & 5.870375000 & -0.051247000 & 0.000012000 \\
\hline 1 & -3.091660000 & -4.194953000 & -0.000095000 & 6 & -4.935737000 & -2.556909000 & 0.000056000 \\
\hline 6 & 3.357175000 & -2.448371000 & -0.000044000 & 6 & -4.335780000 & -3.791592000 & 0.000013000 \\
\hline 6 & 5.968574000 & -1.401395000 & 0.000151000 & 1 & -6.017935000 & -2.476151000 & 0.000104000 \\
\hline 6 & 4.442809000 & -3.286339000 & 0.000048000 & 1 & -4.942075000 & -4.690211000 & 0.000029000 \\
\hline 6 & 5.760503000 & -2.758560000 & 0.000147000 & 1 & -2.463058000 & -4.884997000 & -0.000093000 \\
\hline 1 & 2.349754000 & -2.848833000 & -0.000125000 & 6 & -2.135124000 & -2.778770000 & -0.000074000 \\
\hline 1 & 4.299456000 & -4.361053000 & 0.000045000 & 6 & -2.921226000 & -3.902313000 & -0.000055000 \\
\hline 1 & 6.606272000 & -3.436714000 & 0.000217000 & 1 & -1.055837000 & -2.864714000 & -0.000134000 \\
\hline 1 & 6.978495000 & -1.004162000 & 0.000223000 & 1 & -4.563832000 & 2.058567000 & 0.000097000 \\
\hline 1 & 6.109710000 & 1.273456000 & 0.000179000 & 1 & -5.870375000 & -0.051251000 & 0.000132000 \\
\hline \multicolumn{4}{|c|}{$E=-1152.99692086$} & \multicolumn{4}{|c|}{$E=-1152.99667143$} \\
\hline \multicolumn{4}{|c|}{ NC38 } & & & & \\
\hline 6 & 0.740469000 & 1.698459000 & -0.000200000 & & & & \\
\hline 6 & 1.371129000 & 0.467626000 & -0.000284000 & & & & \\
\hline 6 & -0.740470000 & 1.698459000 & -0.000152000 & & & & \\
\hline 6 & -1.371129000 & 0.467626000 & -0.000195000 & & & & \\
\hline 6 & -0.676972000 & -0.794916000 & -0.000197000 & & & & \\
\hline 6 & -2.642762000 & -2.737349000 & -0.000060000 & & & & \\
\hline 6 & -4.034196000 & -2.699205000 & 0.000064000 & & & & \\
\hline 6 & -1.996881000 & -1.507611000 & -0.000135000 & & & & \\
\hline 6 & -2.677569000 & -0.279942000 & -0.000064000 & & & & \\
\hline 6 & -4.078319000 & -0.204088000 & 0.000040000 & & & & \\
\hline 6 & 0.676973000 & -0.794916000 & -0.000338000 & & & & \\
\hline 6 & -4.761842000 & -1.482131000 & 0.000114000 & & & & \\
\hline 6 & 0.715683000 & 4.136837000 & -0.000036000 & & & & \\
\hline
\end{tabular}




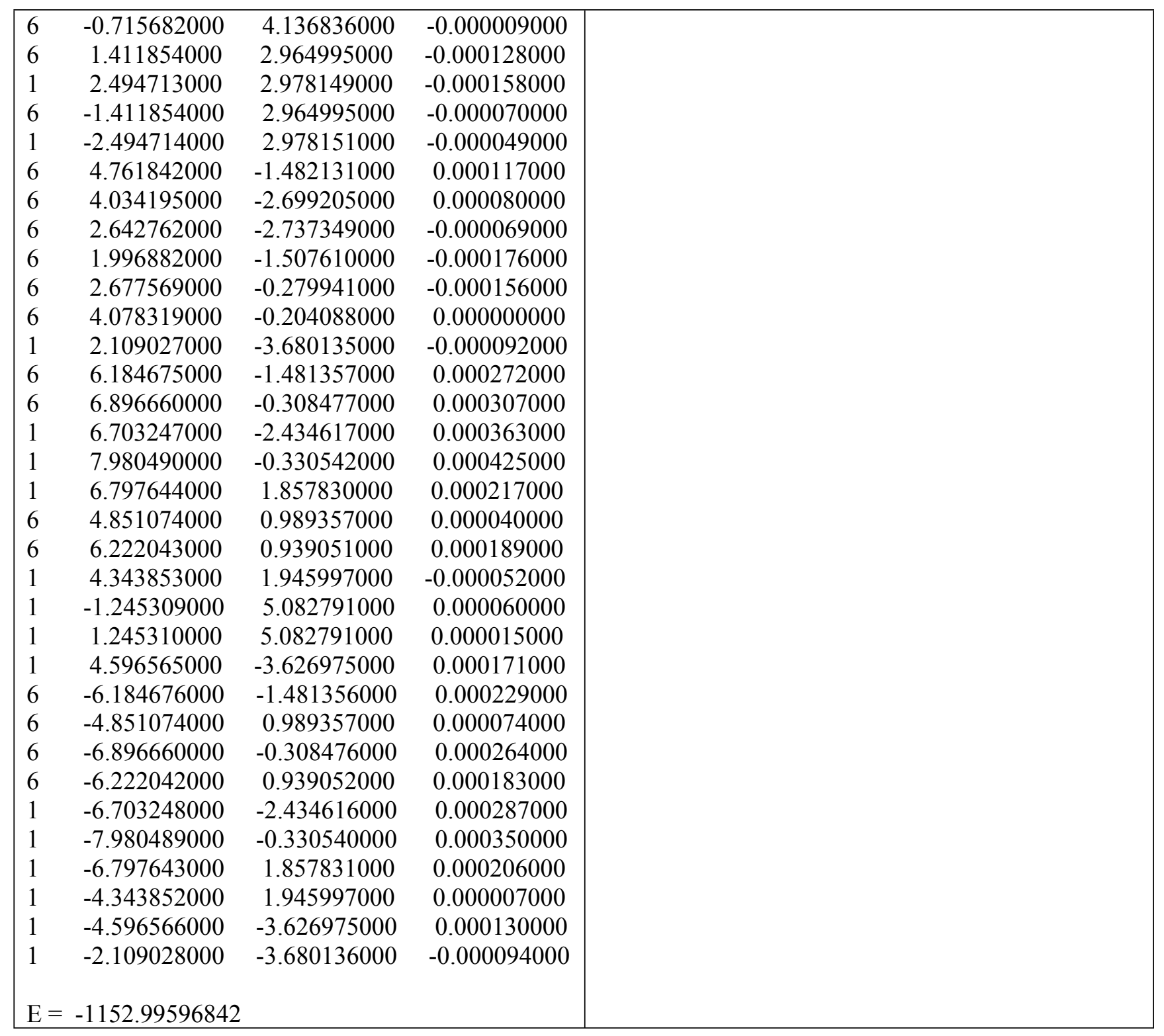

